\title{
44. MAGNETOSTRATIGRAPHIC STUDIES OF SEDIMENTS FROM SITE 744, SOUTHERN KERGUELEN PLATEAU ${ }^{1}$
}

\author{
Barbara H. Keating ${ }^{2}$ and Hideo Sakai ${ }^{3}$
}

\section{INTRODUCTION}

Paleomagnetic studies conducted on board JOIDES Resolution during Leg 119 indicate that the cores collected at Site 744 range from Quaternary through Eocene in age. Initial studies of the sediments completed on board the ship measured the magnetization of the archive halves of the sedimentary cores, using the pass-through cryogenic magnetometer. Stratigraphic plots of the declination and inclination derived from these measurements displayed numerous long intervals with essentially constant magnetic directions. Further study of these intervals led to a discovery that the background signal had been incorrectly computed due to faulty software on the ship. Because this background signal was not recorded in the data-processing system, corrections could not be made. Therefore, subsequent shorebased studies have been made on the individual samples collected at approximately $30-\mathrm{cm}$ intervals in the cores in order to verify the initial magnetostratigraphy reported in the Initial Reports volume for Leg 119 (Barron, Larsen, et al., 1989). Numerous reversals were identified and correlations were suggested with the seafloor magnetic anomaly sequence of Berggren et al. (1985) back to anomaly number 17.

\section{BACKGROUND}

Site 744 is situated on the southern Kerguelen Plateau $\left(31^{\circ} \mathrm{S}\right.$, $80^{\circ} \mathrm{E}$ ) at a water depth of $2307 \mathrm{~m}$. The objective in drilling there was to obtain a reference biostratigraphic and magnetostratigraphic section for the Tertiary. The sediments are pelagic in nature, and the presence of a small amount of calcium carbonate was interpreted as an indication that the site had been situated above the calcium compensation depth (CCD) since the late Eocene. A transition from nannofossil ooze to diatomaceous ooze may reflect "the northward migration of the polar front in the late Miocene" (Barron, Larsen, et al., 1989).

Three holes were drilled at Site 744. Core from Holes 744A and 744B were studied as part of the shipboard analysis using the pass-through cryogenic magnetometer. Core from Hole 744C was reserved for core repository tests and sampling was not permitted. Hole $744 \mathrm{~A}$ sampled the upper $180 \mathrm{~m}$ of the sedimentary sequence; Hole 744B sampled only $80 \mathrm{~m}$. It was difficult to correlate the two reversal sequences due to the software analysis problem, which left many indeterminate sections-particularly within the Hole $744 \mathrm{~A}$ reversal sequence. The reversal sequence derived from the shipboard analysis was a composite of the records from both Holes 744A and 744B.

\footnotetext{
${ }^{1}$ Barron, J., Larsen, B., et al., 1991. Proc. ODP, Sci. Results, 119: College Station, TX (Ocean Drilling Program).

2 Hawaii Institute of Geophysics, University of Hawaii, Honolulu, Hawaii 96822, U.S.A.

${ }^{3}$ Department of Earth Sciences, Toyama University, Gofuku 3190, Toyama City 930, Japan.
}

\section{LITHOSTRATIGRAPHY}

The sediments sampled at Site 744 consist of white nannofossil and diatom oozes. The sediments are characterized by a high (usually greater than $90 \%$ ) biogenic content. Foraminifers, radiolarians, silicoflagellates, and sponge spicules account for $5 \%-20 \%$ of the sediments. The sediments have been divided into two lithologic units. Unit I consists of a diatom ooze and Unit II consists of a nannofossil ooze.

\section{Unit I-Diatom Ooze}

Unit I was sampled in Sections 119-744A-1H-1 through 119744A-3H-CC and in Sections 119-744B-1H-1 through 119-744B$3 \mathrm{H}-\mathrm{CC}$. Unit I is a white ooze. The amount of siliceous and calcareous components fluctuates, as is reflected by sporadic pale intervals. The discrete samples collected for paleomagnetic study have uniform color and texture. Micrite is present throughout all but the lower $5 \mathrm{~m}$ of this unit. The micrite content varies from $40 \%$ to $15 \%$, decreasing downward in Hole $744 \mathrm{~A}$. The sedimentologists report (Barron, Larsen, et al., 1989) that the detrital component of the sediment is almost negligible. However, coarse sand grains, granules, and small pebbles up to $5 \mathrm{~cm}$ in diameter occur throughout this unit, varying from a trace to $2 \%$. The clasts include: quartz, lithic fragments, granite, quartzfeldspar-biotite gneiss, and amphibole. These detrital components, despite making up only a small percentage of the sediments, can greatly affect the magnetization of individual samples. Multicomponent magnetizations are often associated with a fine grained matrix surrounding detrital clasts.

\section{Unit II-Nannofossil Ooze}

Unit II was sampled in Sections 119-744A-4H-1 through 119744A-20H-CC and in Sections 119-744B-4H-1 through 119744B-9H-CC. Unit II consists of white nannofossil ooze. The colors of the paleomagnetic samples appear monotonous. Calcareous nannofossils constitute the majority of the sediments, generally between $70 \%$ and $85 \%$. Foraminiferal tests constitute between $3 \%$ and $5 \%$ generally, but can range up to $15 \%$. Radiolarians, silicoflagellates, and sponge spicules are commonly absent or appear in trace abundances. Nonbiogenic components are minor. Scattered coarse sand grains and granules were found down to Section 119-744A-4H-3, $100 \mathrm{~cm}$. Farther down the hole, manganese oxide-coated grains are present. In Core 119$744 \mathrm{~A}-17 \mathrm{H}$, most of the coarse, coated grains are concentrated along the core liner and, in some places, drilling disturbance was observed. Thus, it was suggested (Barron, Larsen, et al., 1989) that all the coarse fraction in Section 119-744A-20H was due to downhole contamination. Manganese oxide streaks and fragments of submillimeter size are disseminated throughout the unit. Round to oval halo structures with diameters up to 10 $\mathrm{cm}$ have also been described within Unit II. It was suggested that the material being leached could be the manganese, but conclusive laboratory studies were not made (Barron, Larsen, et al., 1989). The observations are of concern because iron oxides 
are usually mobilized in diagenetic processes that mobilize manganese (Malcolm and Stanley, 1982). Diagenesis of this nature can produce important secondary components of magnetization (referred to as multicomponent magnetization later in this text) that are acquired after the rocks are formed.

\section{PALEOMAGNETIC METHODS AND INSTRUMENTATION}

Twelve samples were selected from the larger collection of samples for initial paleomagnetic studies. These samples were demagnetized in an alternating field in multiple steps to $60 \mathrm{mT}$. The results of these demagnetizations were then compared using stereographic projections, orthogonal plots, and demagnetization curves. The majority of the samples are characterized by stable magnetization at demagnetization levels above $10 \mathrm{mT}$; thus blanket demagnetizations were then carried out on all of the samples at $15,22.5$, and $30 \mathrm{mT}$.

\section{Results of Alternating Demagnetization}

All of the samples used in this study were stored within a doubly shielded mu-metal room for 1 month prior to measurement in order to remove low coercivity VRM (viscous remanent magnetization) components. The magnetization was measured on a ScT (Super Conducting Technology) three axis cryogenic magnetometer. The holder magnetization was measured periodically and subtracted from the specimen moment. The demagnetizations were carried out within the mu-metal shielding using a three-axis tumbler system driven by a Schonstedt demagnetizer. The samples remained within the shielded room throughout the demagnetization sequence.

Most samples collected in this study were stably magnetized. The majority displayed simple decay to the origin of a orthogonal plot upon demagnetization in alternating fields of 15 and 30 $\mathrm{mT}$ (Fig. 1). After demagnetization to $5 \mathrm{mT}$, the magnetic directions and intensity decay toward the origin of the orthogonal plot. This is typical of the most-stably magnetized samples. Some samples showed a small component of secondary magnetization, which was removed by the first demagnetization step at $15 \mathrm{mT}$, but are otherwise very stably magnetized (Fig. 2). After demagnetization at 5-15 $\mathrm{mT}$, the directions and intensity decay univectorially to the origin of the diagram. Stereographic projections showing the change in direction on demagnetization and demagnetization curves for these stably magnetized samples are shown in Figures 3 and 4. Since the majority of the samples are stably magnetized by a single component of magnetization, these samples are interpreted as being recorders of earth's polarity at the time of deposition.

A second group of samples displayed significant change in magnetic direction on alternating demagnetization. Examples of this magnetization are shown in Figure 5. Most of these samples have natural remanent magnetization (NRM) directions characterized by shallow inclinations (less than $45^{\circ}$ ). Upon demagnetization, these samples show changes in polarity, but remain at shallow inclinations. Roughly half of these samples are located at polarity transitions and it is likely that within this group of samples there is a physical superposition of polarity within discrete samples. If additional time had been available, these samples would have been sliced into wafers and remeasured in order to verify that a physical superposition of magnetization was present.

The remaining samples in this group are characterized by two components of magnetization. The primary magnetization is of one polarity, and the secondary magnetization appears to be of the opposite polarity. Within this hole, leaching of Mn oxides was observed (Barron, Larsen, et al., 1989). These observations raise concerns about the fidelity of the magnetic record. When Mn oxides are mobilized, Fe oxides are generally mobilized
(Malcolm and Stanley, 1982). These oxides could produce the secondary magnetization observed within isolated samples within this hole.

The presence of a substantial number of samples with multicomponent demagnetization behavior and low inclinations in a section in which most samples are characterized by single component magnetization and high inclinations may well be explained by superimposed magnetizations of the opposite polarity. This superposition could result from sampling a polarity transition, from secondary growths of iron oxides with sediments associated with Mn-oxide mobilization or it might result from heterogeneities within these sediments. Detrital fragments derived from metamorphic rocks as well as manganese oxide coated grains (downhole contamination) are described in the site report (Barron, Larsen, et al., 1989). Samples containing detritus may have a fine grain matrix of one polarity and contain coarse detrital grains magnetized in a random direction, which appears to be a component of the opposite polarity. A list of samples which display transitional or multicomponent magnetization is given in the Appendix. Stratigraphic plots of the sample inclinations from the NRM, $15 \mathrm{mT}, 22.5 \mathrm{mT}$, and $30 \mathrm{mT}$ demagnetization steps are shown in Figure 6. Listings of the sample directions are also given in the Appendix. In order to insure the fidelity of the magnetostratigraphic results, all samples which display transitional directions or multicomponent magnetization have been omitted from the polarity determinations (Figs. 7 and 8 ). The stratigraphic plots show that very little direction change occurs in most samples upon demagnetization. Therefore, the reversal sequence should be reliable since it is based solely upon the selected data consisting of only samples which displayed stable single component magnetization.

\section{REVERSAL SEQUENCE}

The majority of the samples studied display single component magnetization and high paleoinclinations between $70^{\circ}$ and $85^{\circ}$ (positive and negative). Thus, the designation of polarity units is in general very obvious and straightforward. Since this site is situated in the Southern Hemisphere, normally magnetized rocks have negative inclination values. In Figure 9, polarity designations (black $=$ normal polarity; white $=$ reversed polarity) are shown. No re-orientation of cores was attempted, so declinations have not been used in designating polarity. In all, 45 polarity zones were identified.

A comparison between the sampling interval for discrete samples measurements made in the shore-based laboratory and those made using a Molespin spinner magnetometer aboard ship indicates that there is good agreement between the two sets of measurements. When the shorebased results are compared to the shipboard cryogenic magnetometer measurements of continuous cores we also find good general agreement, although it is obvious that the reversal boundaries have been smeared out or perhaps obscured by use of the shipboard records (where the measurements are integrated over more than $10 \mathrm{~cm}$ ).

ODP has a policy of limiting paleomagnetic sampling to two samples per core section. During this leg, additional sampling was requested but only three samples per section were permitted. Routine magnetostratigraphy studies of deep sea cores generally utilize a sampling interval of $10 \mathrm{~cm}$. Between 40 and 180 mbsf, the shipboard cryogenic magnetometer measurements detected 95 polarity changes. With the discrete sample study described here roughly half that number were identified. The biostratigraphic horizons identified in the Initial Reports (Barron, Larsen, et al., 1989) indicate this interval ranged in age from 10 to roughly $40 \mathrm{Ma}$. On average, three polarity reversals should be expected within each million year interval. With a sampling interval of only three samples per core section, or roughly $35 \mathrm{~cm}$, only one sample would be expected per polarity unit. The dis- 
$119-740 \mathrm{~A}-10 \mathrm{H}-5,62 \mathrm{~cm}$

100

215

322

430

Div. $=2.5 \mathrm{mT}$

$1 \mathrm{~A}$

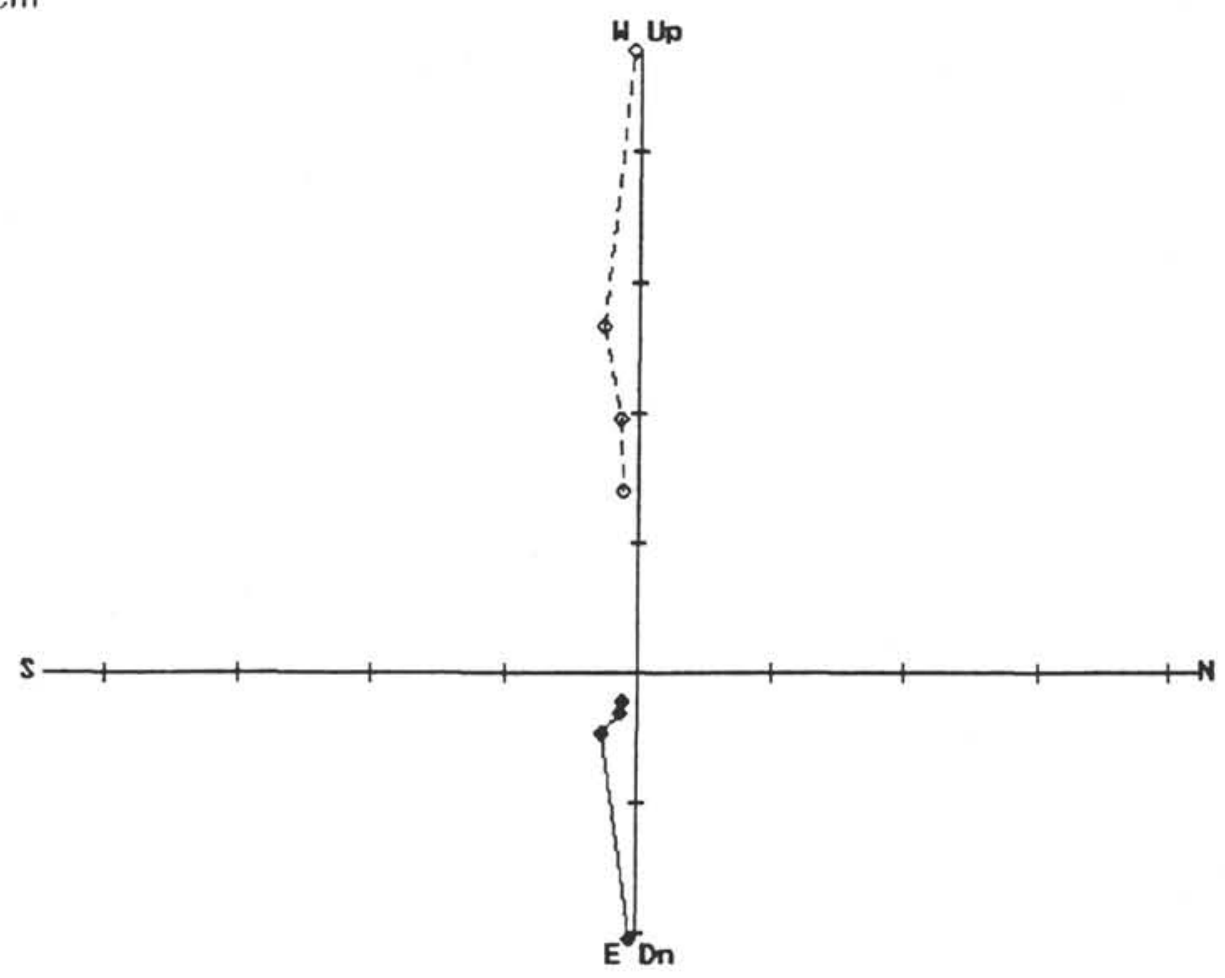

$119-744 \mathrm{~A}-10 \mathrm{H}-3,53 \mathrm{~cm}$

105

210

330

450

Div $=1.0 \mathrm{mT}$

$1 \mathrm{~B}$

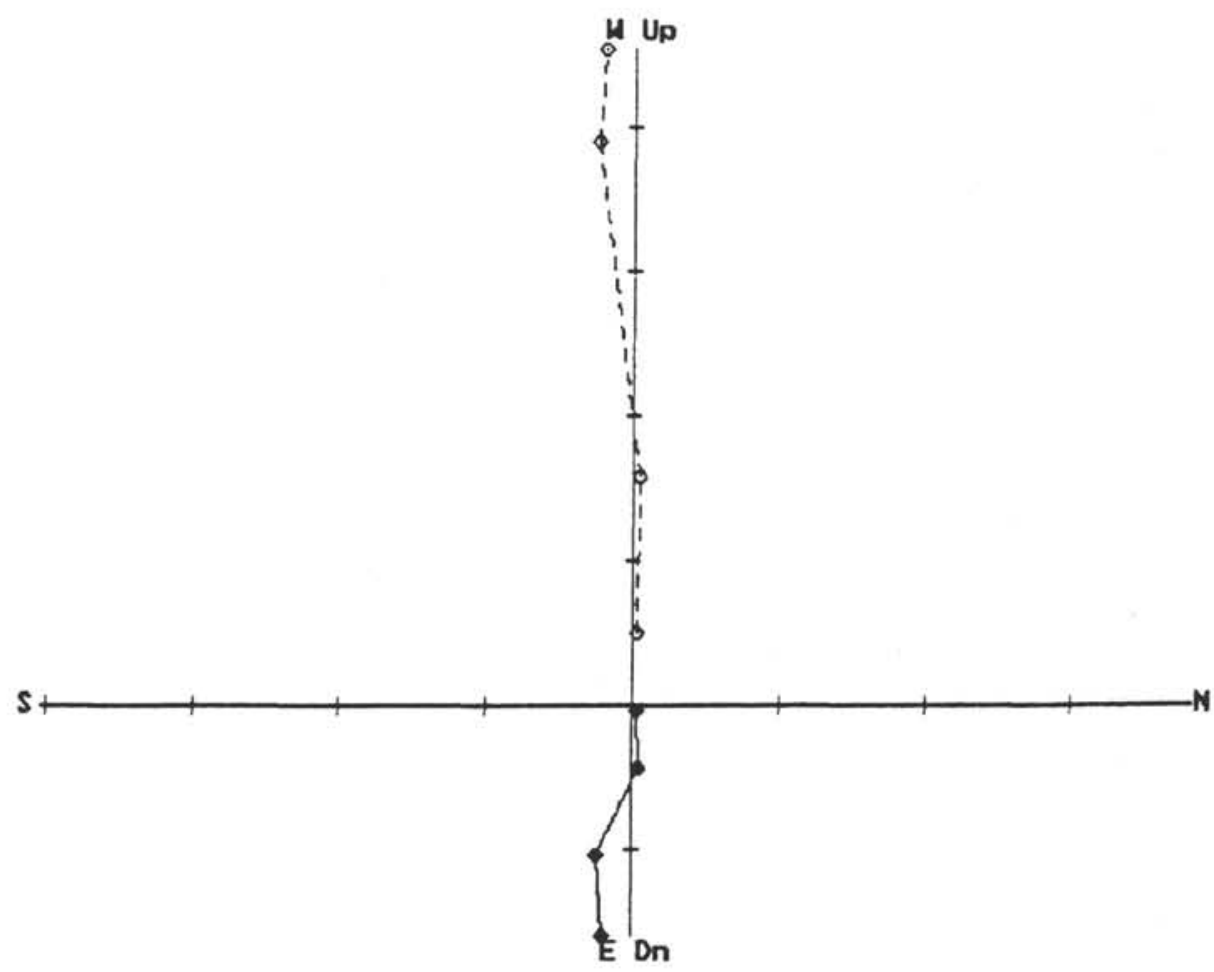

Figure 1. Orthogonal plots of the vector decay on demagnetization for two stably magnetized samples. The samples show a simple univectorial decay toward the origin. The demagnetization levels are indicated in milliTesla (mT). 
B. H. KEATING, H. SAKAI

119-744A-19H-2, $80 \mathrm{~cm}$

100

205

310

420

530

640

750

Div $=2.0 \mathrm{mT}$

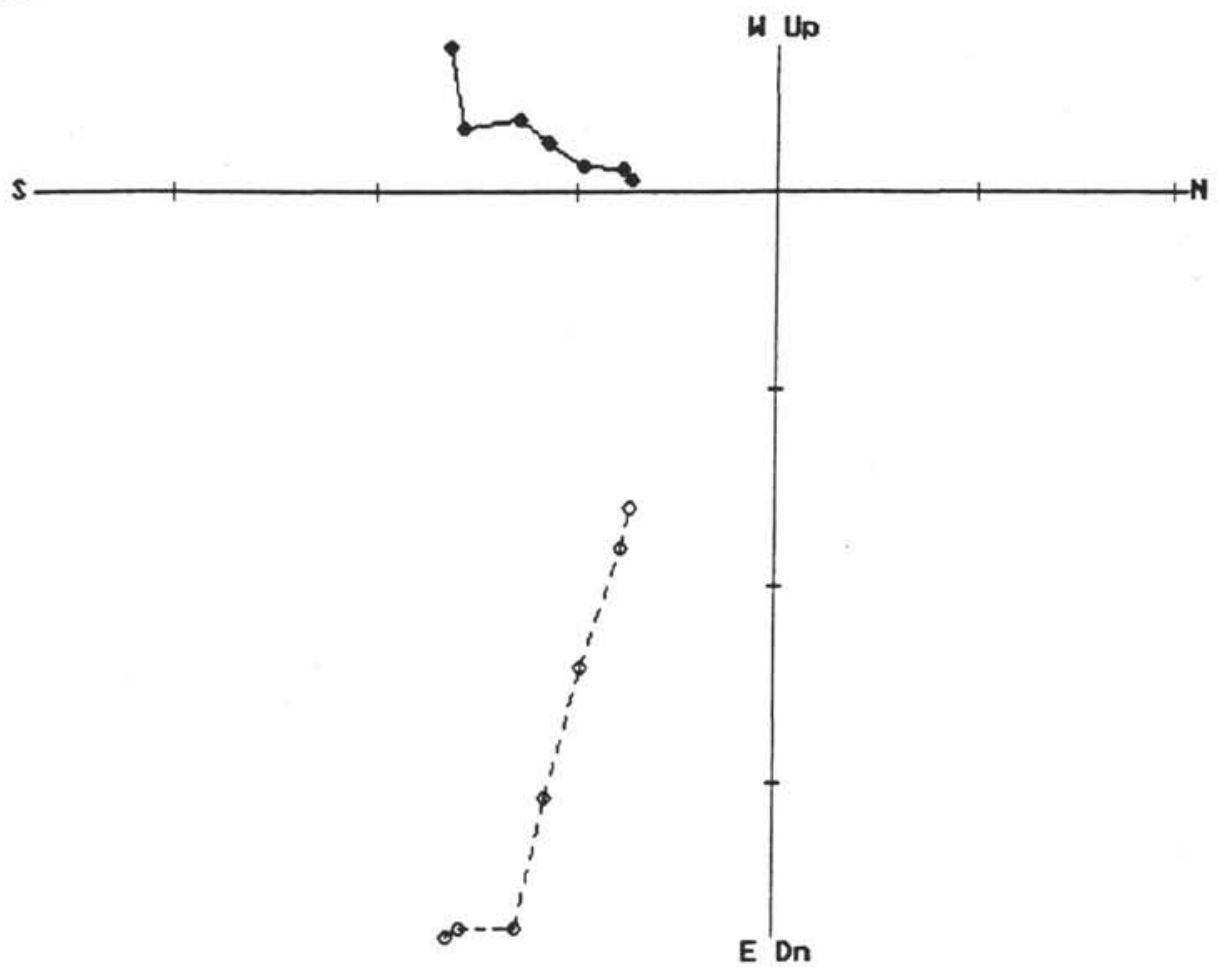

119-744A-16H-3, 80cm

100

215

322

430

Div $=2.0 \mathrm{mT}$

$2 B$

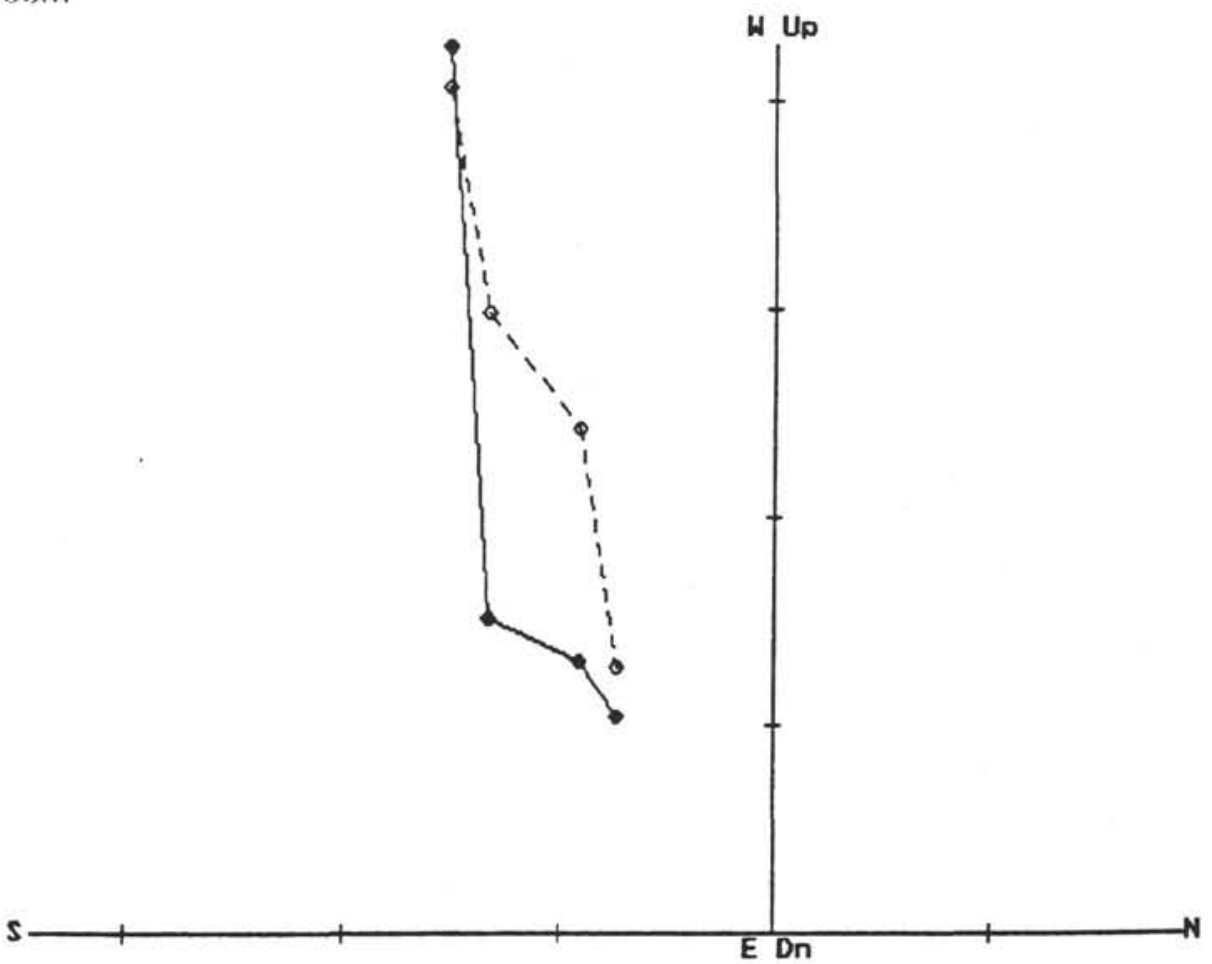

Figure 2. Orthogonal plots of samples showing an initial secondary component of magnetization removed between 0 and 05 or 0 and 15 $\mathrm{mT}$. After further demagnetization, the magnetization decays toward the origin of the orthogonal plot. 
119-744A-6H-2, 44cm
100
215
322
430

$3 \mathrm{~A}$

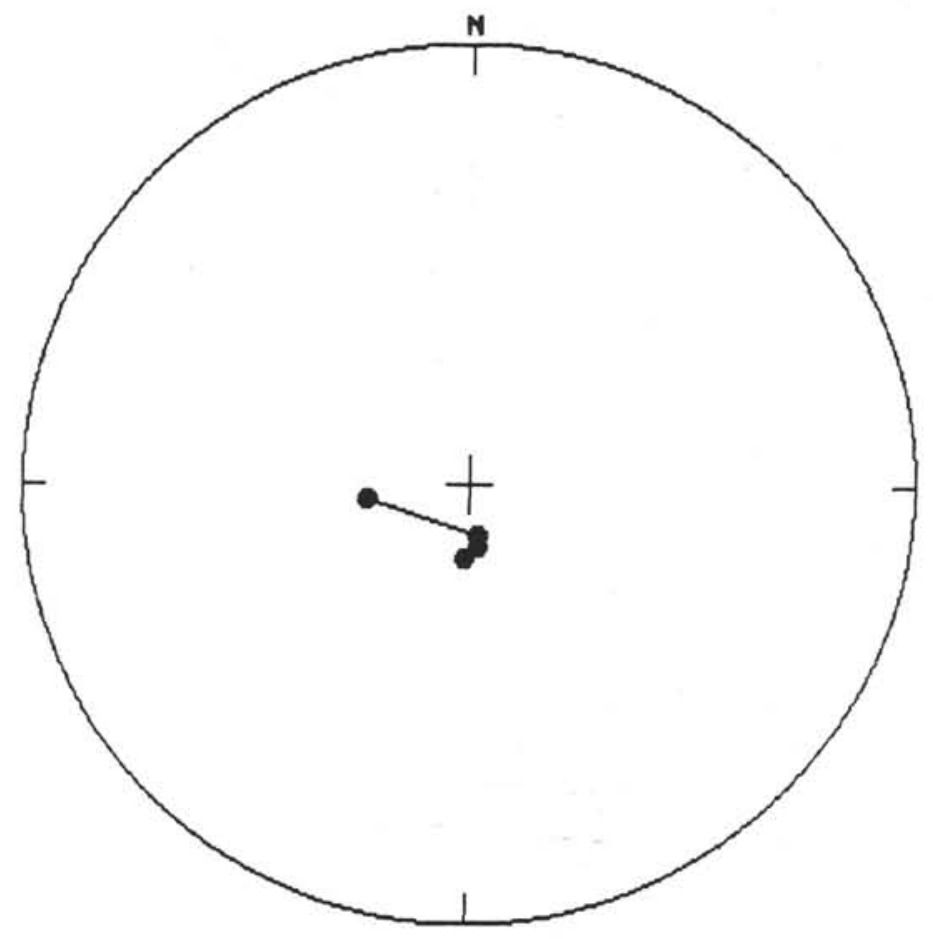

119-744A-15H-1, $24 \mathrm{~cm}$

100

205

310

430

540

650

$3 B$

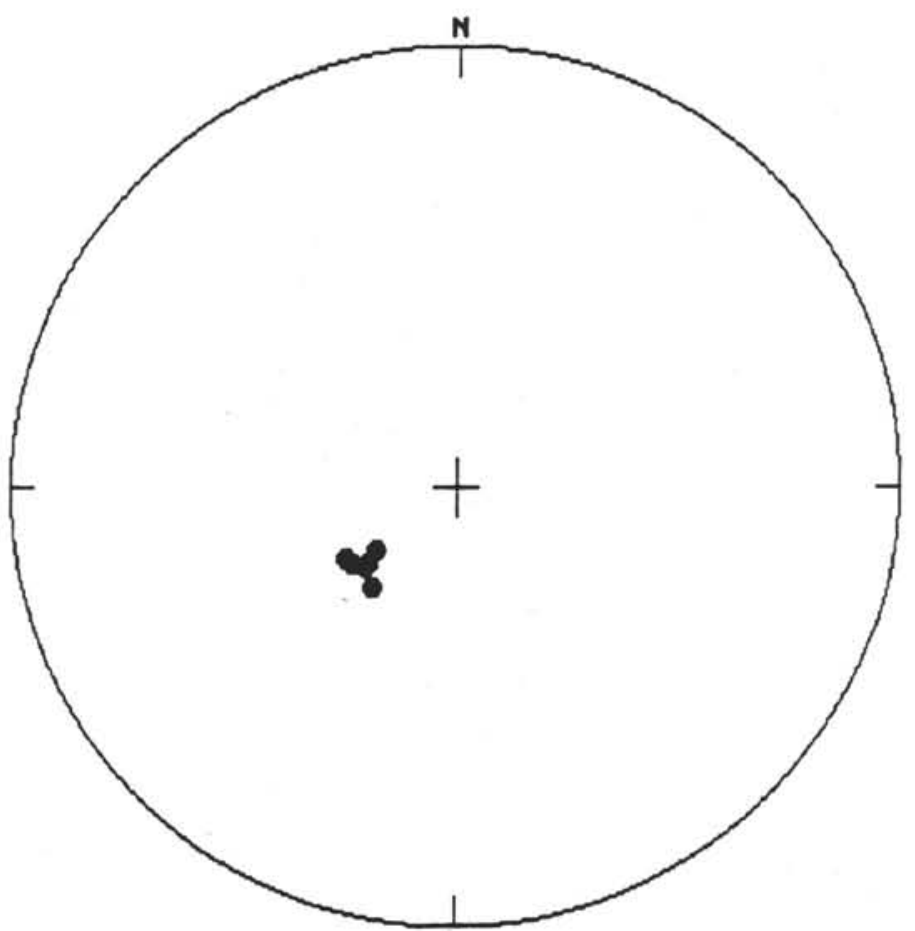

Figure 3. Stereonet projections of sample directions. These stably magnetized samples show very little change in direction after demagnetization to 5 or $10 \mathrm{mT}$. 
B. H. KEATING, H. SAKAI

119-744A-10H-4, 65cm
110
220
330
440
550

$4 \mathrm{~A}$

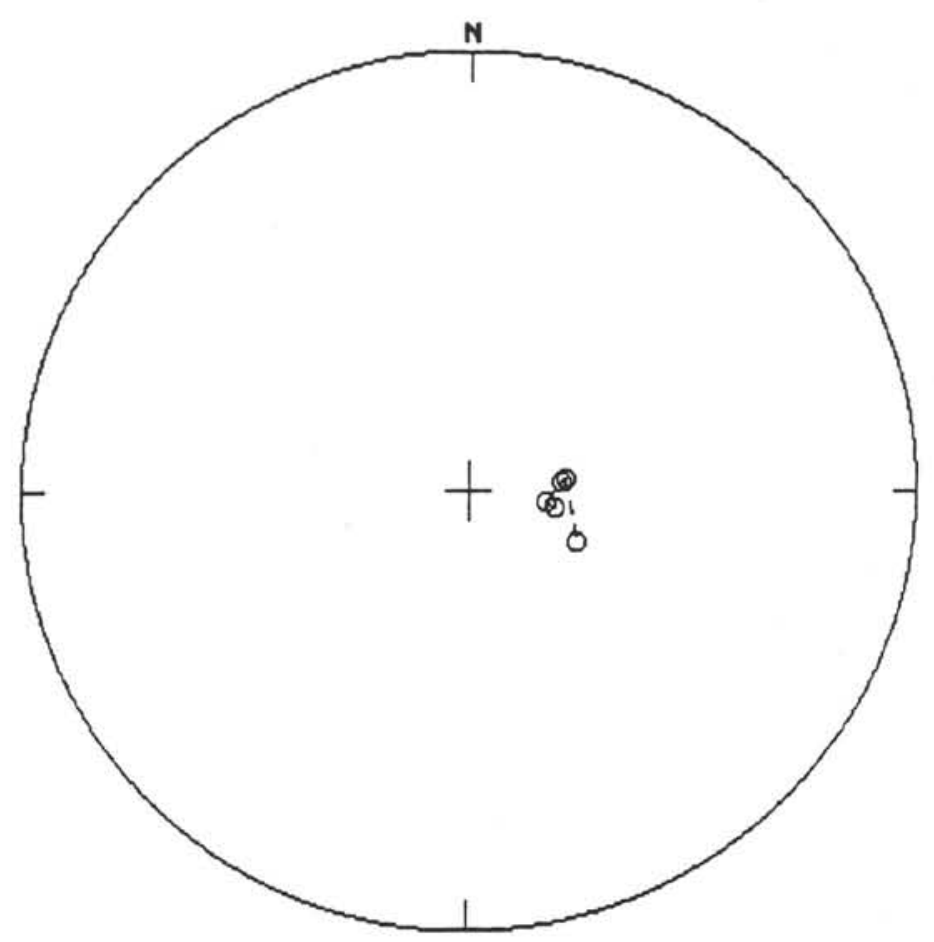

119-744A-10H-3, 53cm
105
210
330
450

4B

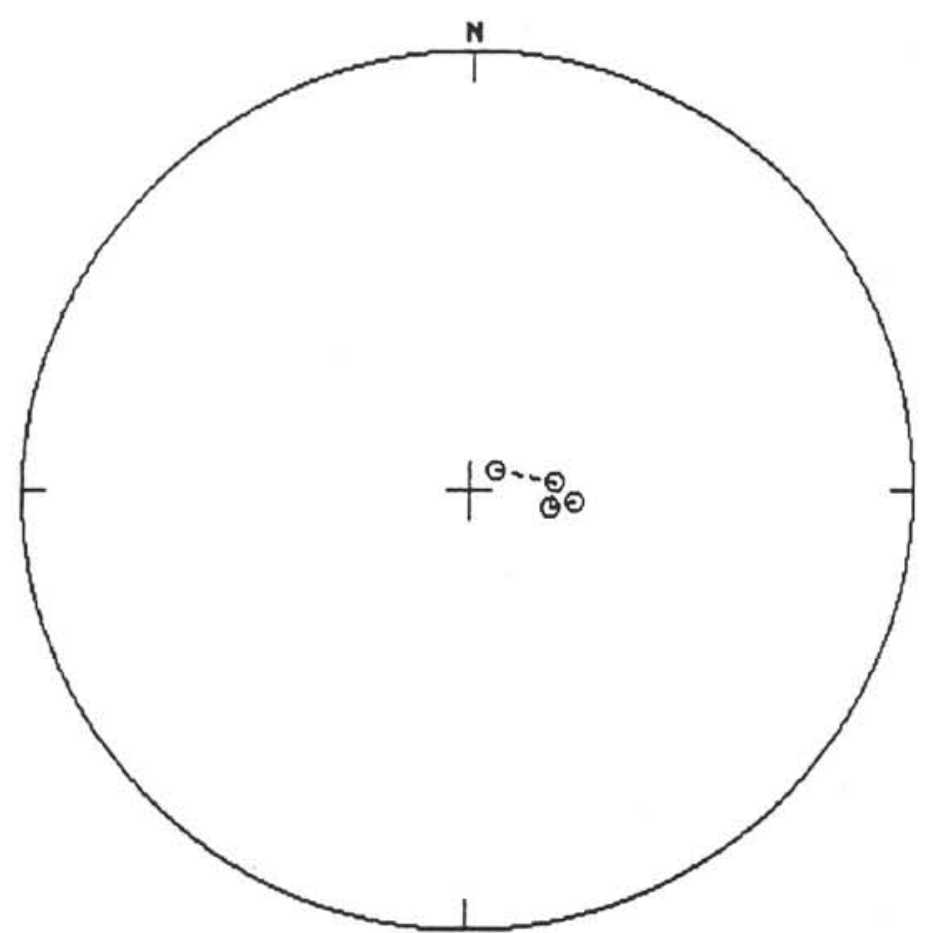

Figure 4. Stereonet projections of sample directions with demagnetization to $35 \mathrm{mT}$. A small component of secondary magnetization is removed between 0 and $15 \mathrm{mT}$. The directions remain very stable after this small secondary component has been removed. 
119-744A-11H-5, $120 \mathrm{~cm}$

100

205

310

420

530

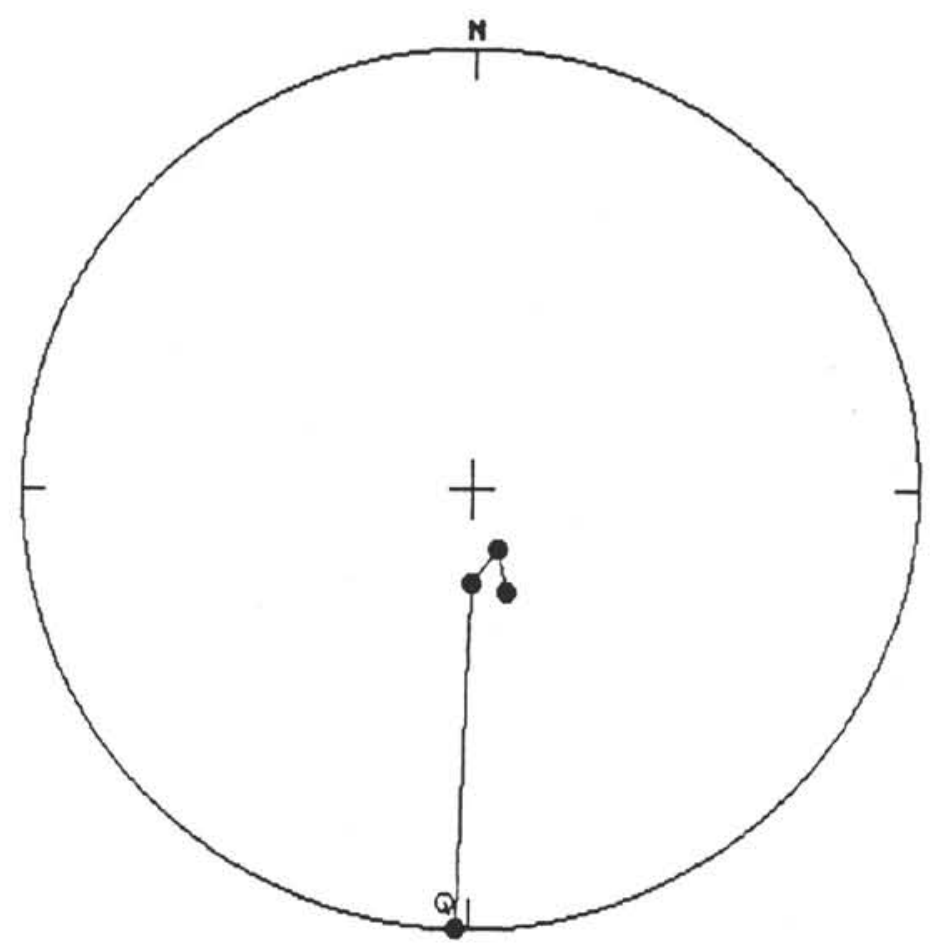

119-744B-7H-7, 64cm
100
222
330

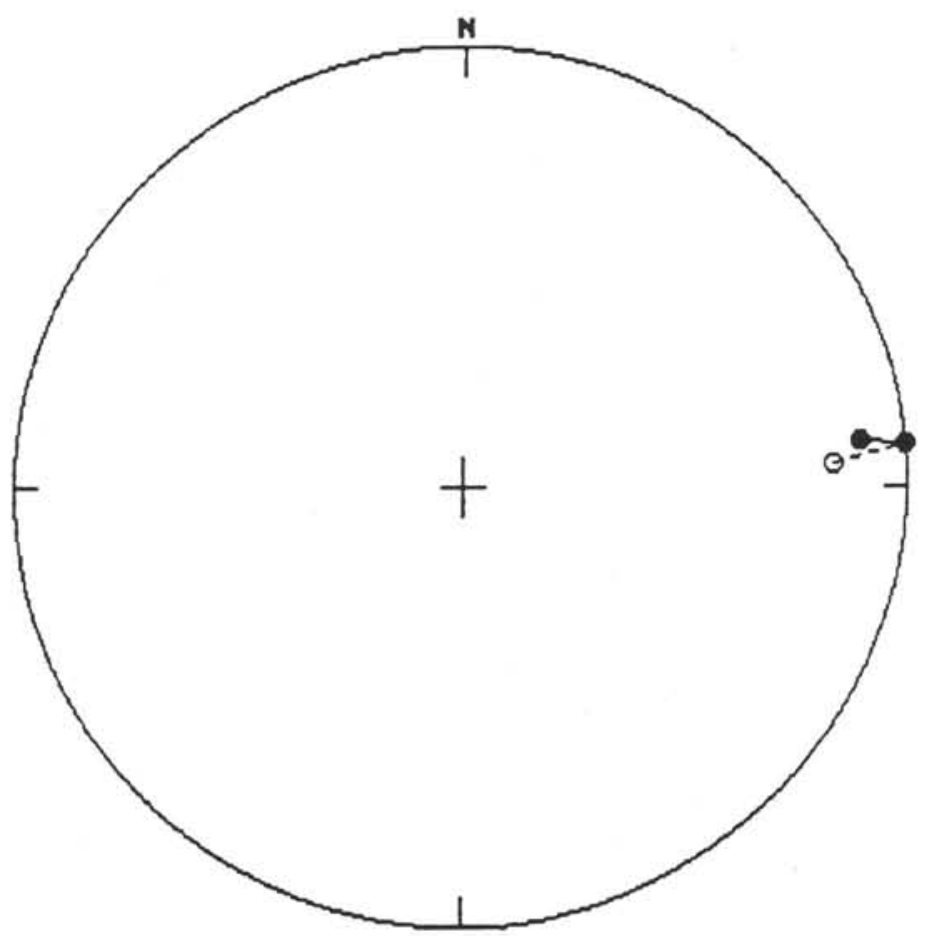

Figure 5. Stereonet projections of anomalous or transitional samples which change polarity upon demagnetization. 


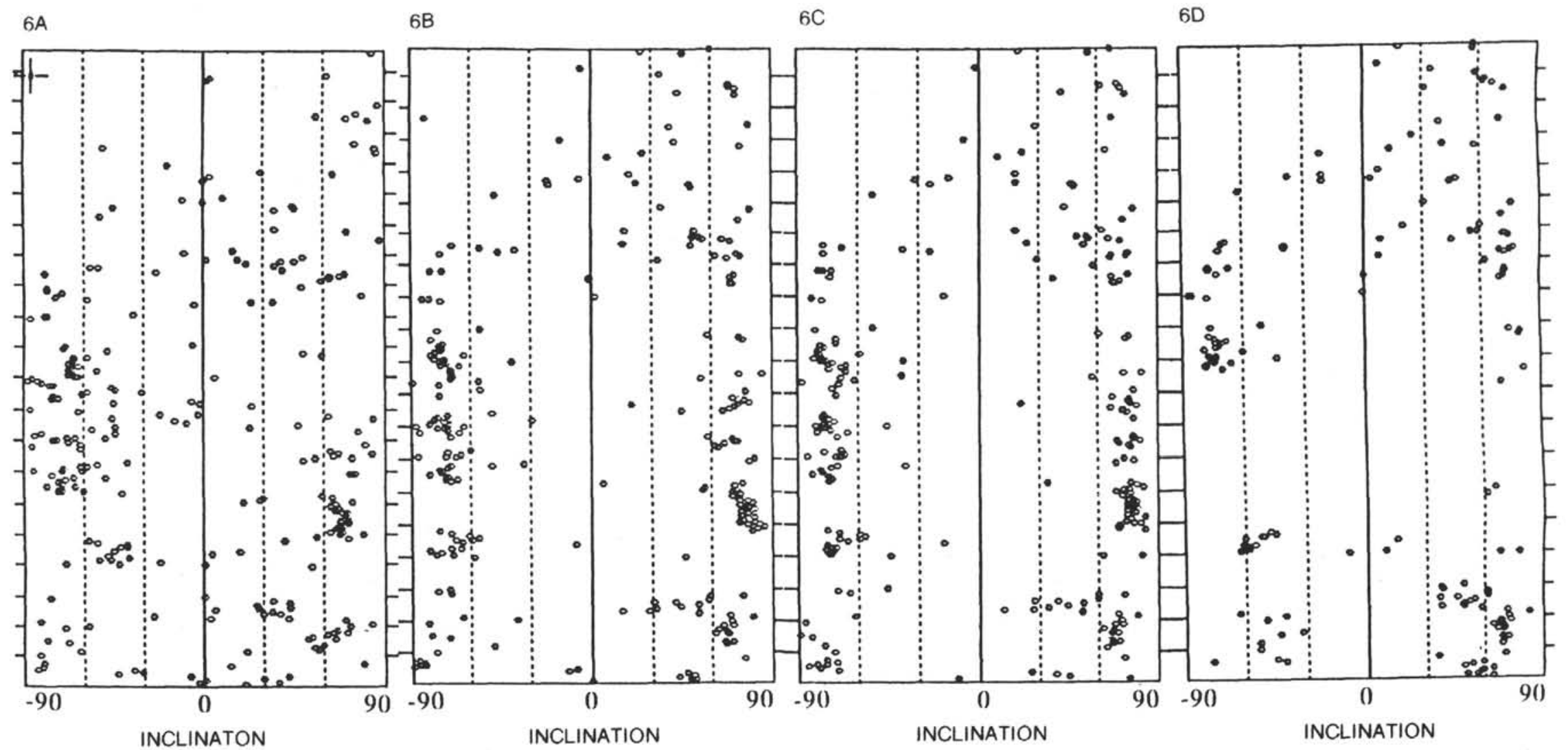

Figure 6. Comparison of stratigraphic plots of sample inclinations. The column at left is the natural remanent magnetization. Results after demagnetization at the $15,22.5$, and $30 \mathrm{mT}$ steps are shown progressing toward the right in the figure. 


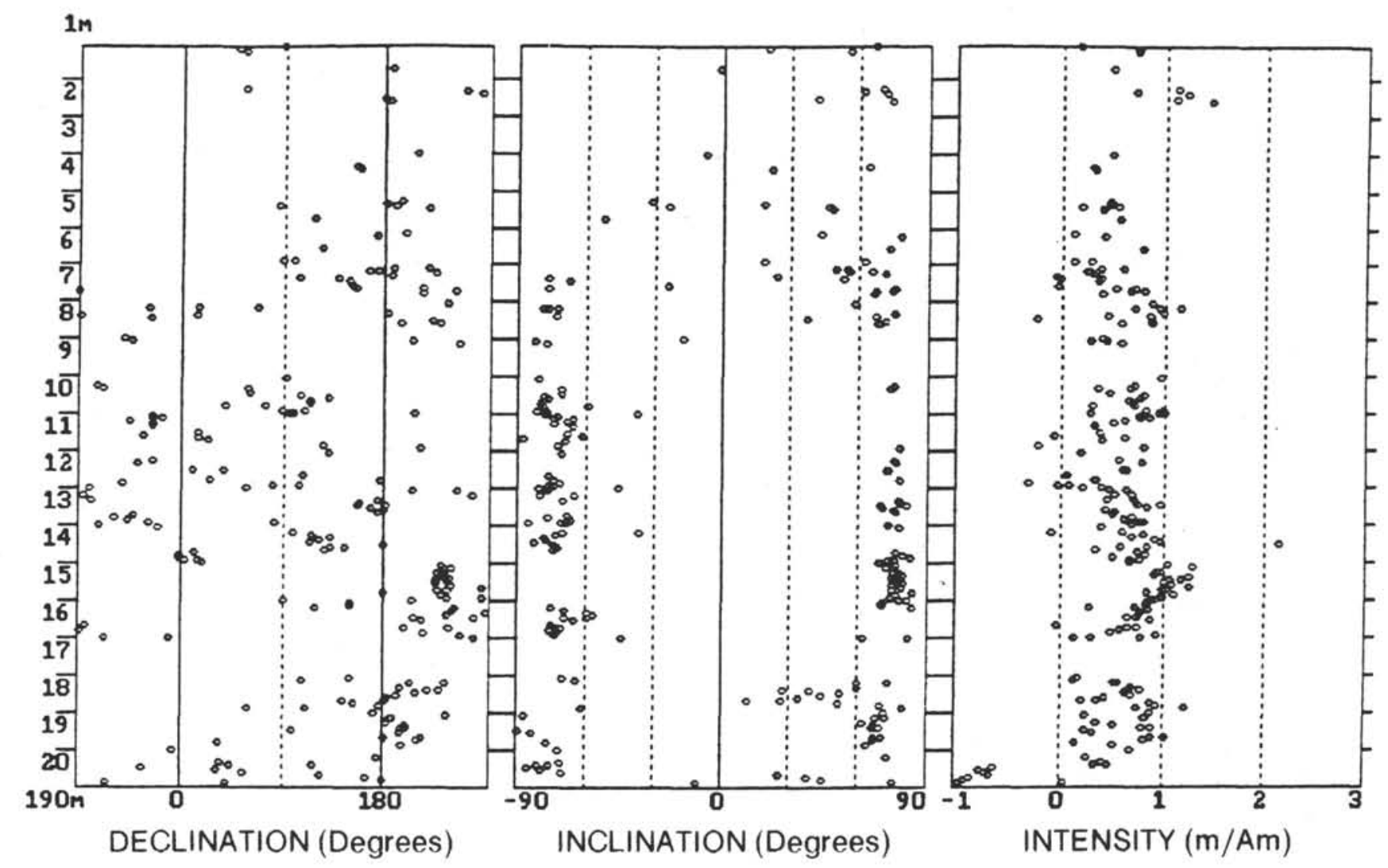

$7 A$

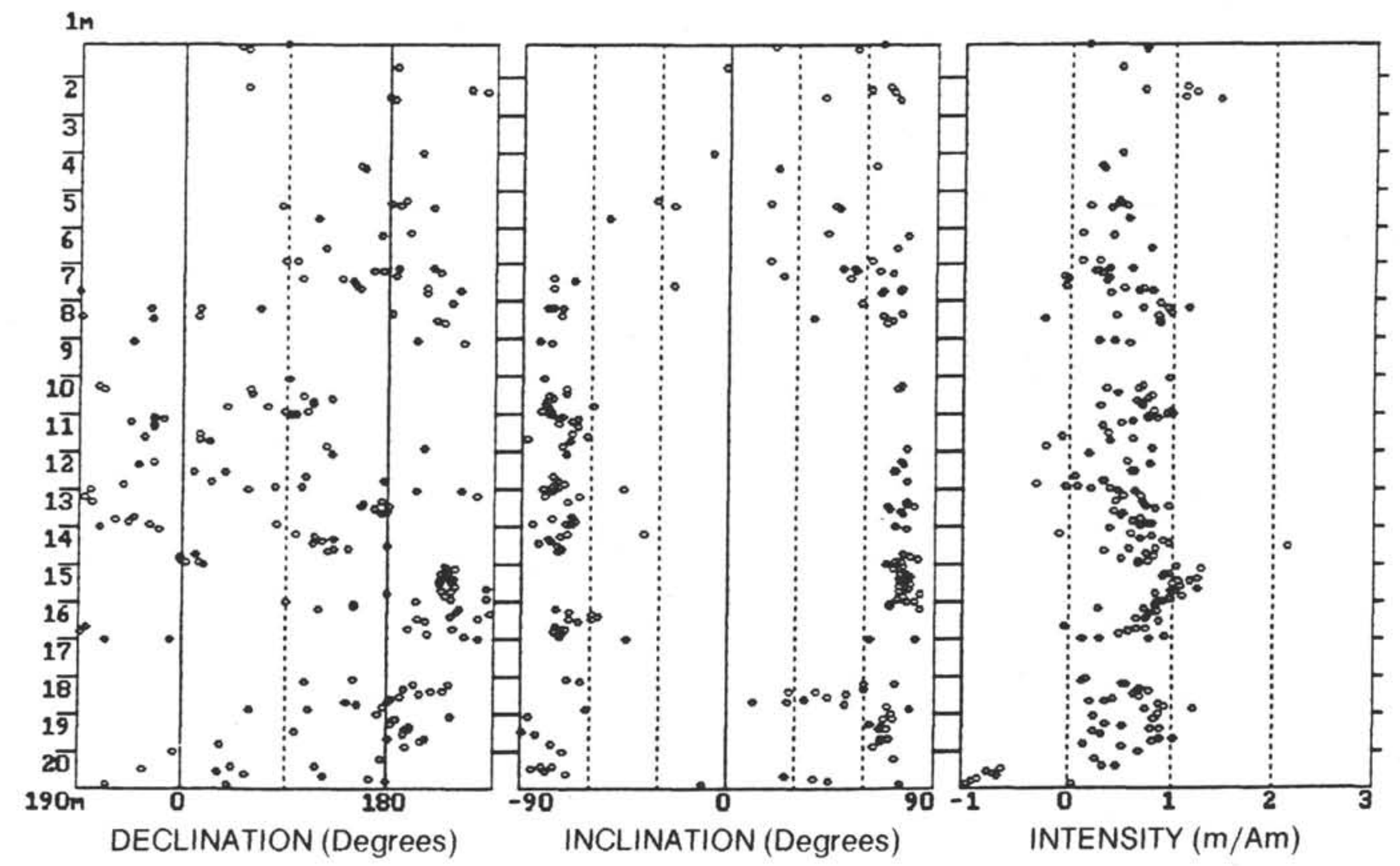

Figure 7. Comparison of stratigraphic plots of sample declinations, inclinations, and intensity after demagnetization to $22.5 \mathrm{mT}$ for samples from Hole $744 \mathrm{~A}$. All the samples are shown in the upper part of the figure. The selected data with transitional or anomalous unstably magnetized samples removed are shown at the bottom. 

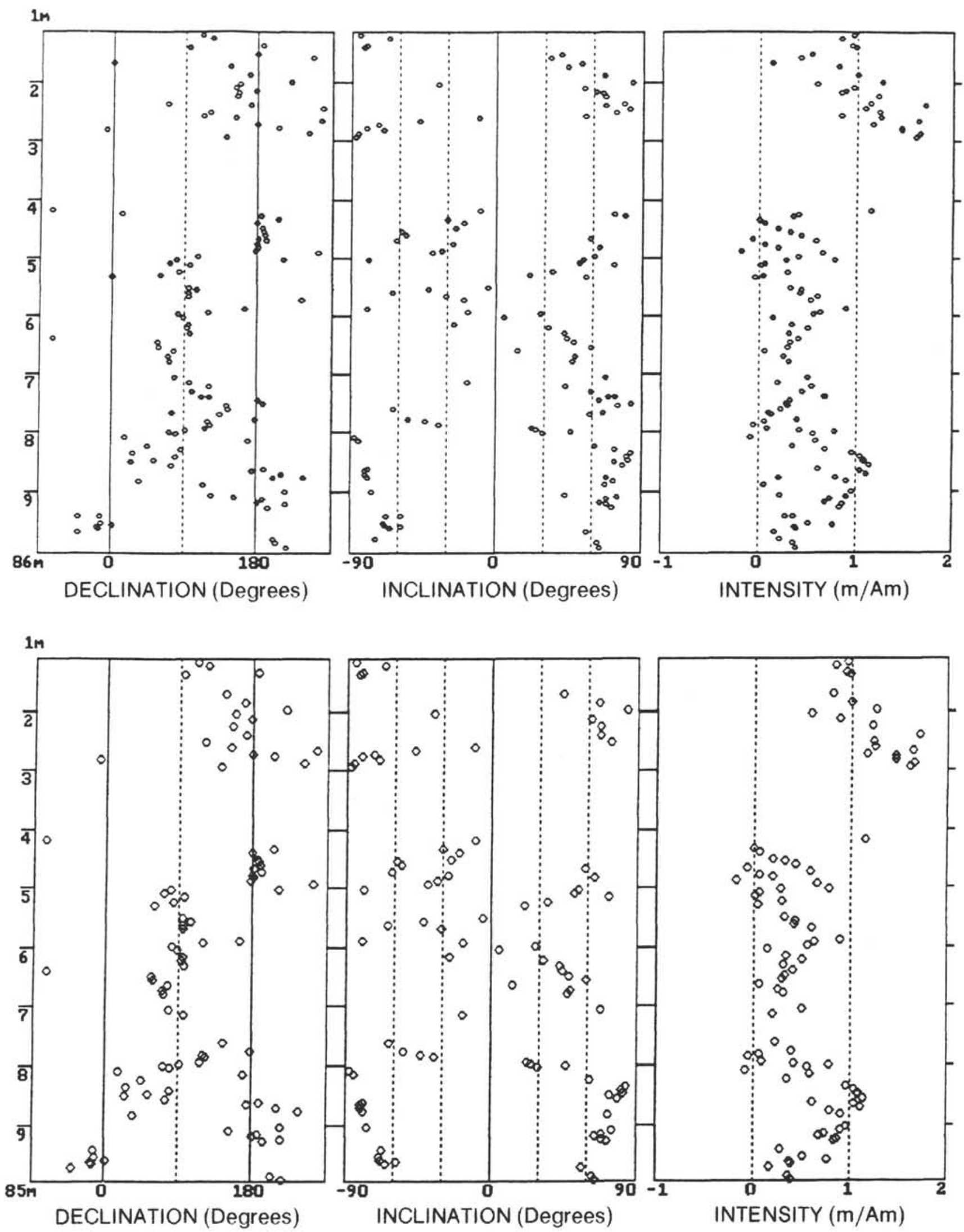

Figure 8. Comparison of stratigraphic plots of sample declinations, inclinations, and intensity after demagnetization to $22.5 \mathrm{mT}$ for samples from Hole $744 \mathrm{~B}$. All the samples are shown in the upper part of the figure. The selected data with transitional or anomalously magnetized samples removed are shown at the bottom. 


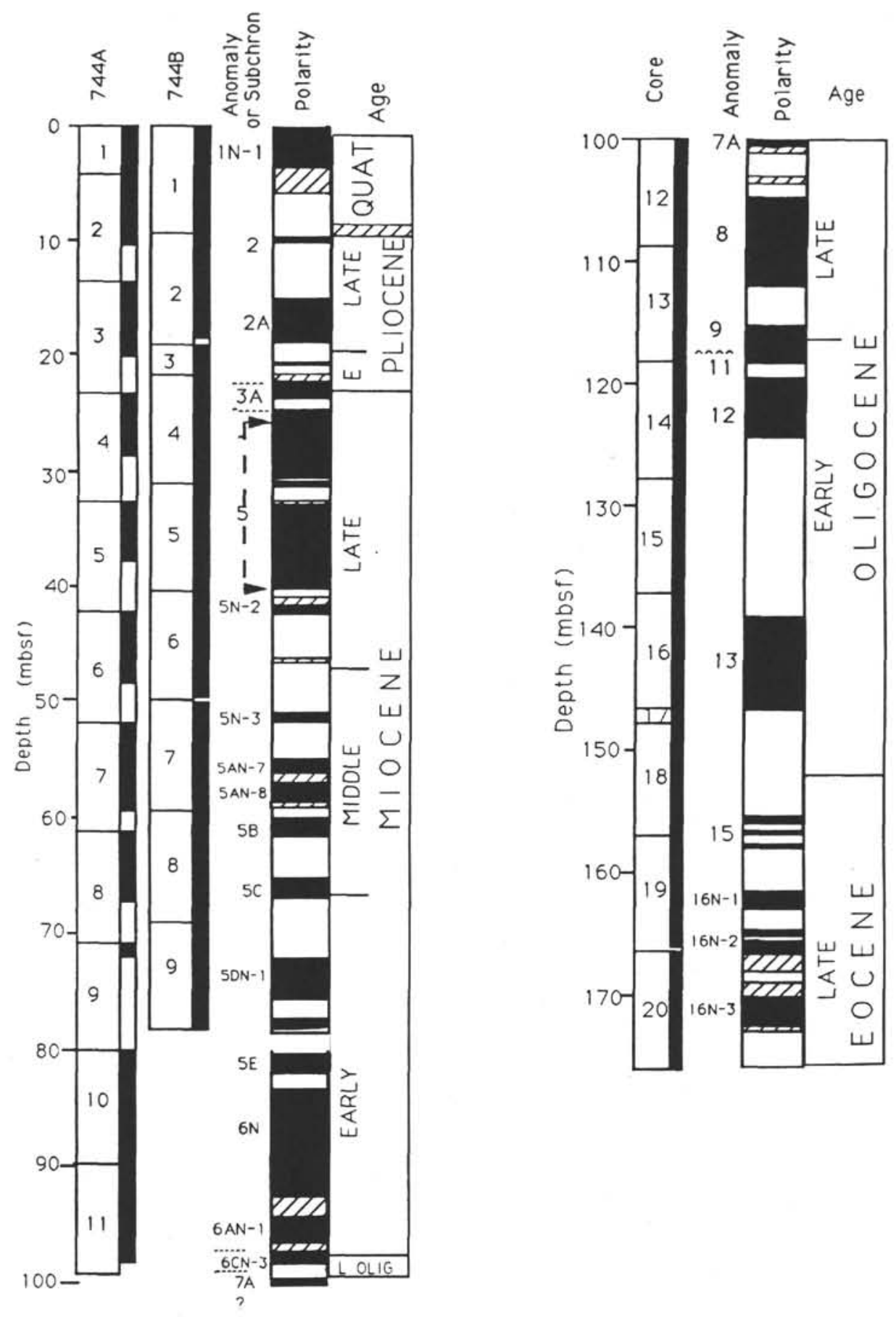

Figure 9. Comparison of the composite magnetic polarity sequence from Holes $744 \mathrm{~A}$ and $744 \mathrm{~B}$ produced from the shipboard cryogenic results and compared to the reference sequence of Berggren et al. (1985). The tie lines between the observed reversal stratigraphy and the reference sequence are based upon the biostratigraphic zonations described in the Initial Reports (Barron, Larsen, et al., 1989).

crete sampling in Hole 744 has greatly undersampled the polarity sequence in this hole. Normally, the stratigraphic thickness of a polarity zone reflects the chronologic duration of a polarity zone. Due to the severe sampling restrictions a simple correlation of varying thickness of reversals to the geomagnetic time scale, proved a very difficult task. Therefore, the correlations were made based upon age constraints derived from biostratigraphic and isotope studies. These studies are briefly outlined here.

\section{BIOSTRATIGRAPHY CONSTRAINTS}

The details of the fossil zonations and biostratigraphic correlations are discussed by Barron et al. (this volume). Core recovery within the interval $0-78.5$ mbsf within Hole 744B was nearly $100 \%$, thus the biostratigraphic and paleomagnetic correlations for the first 78.5 mbsf were based upon the fossils and reversal sequence observed in Hole 744B. The assignment of magnetic polarity chrons and subchrons is constrained by microfossil da- 
tum levels (Barron et al., this volume), oxygen isotope events (Woodruff and Chambers, this volume), and strontium isotope values (Barrera at al., this volume) which are listed in Table 5 of Barron et al. (this volume). The stratigraphic sequence between 0 and 20 mbsf in Hole 744B is assigned to the Brunhes Normal Polarity Chron through the Gauss Normal Polarity Chron, corresponding to seafloor anomalies 1,2 , and $2 \mathrm{~A}$.

Based upon the radiolarian zonations, first occurrences of diatoms, and the sequence of reversals, a gap in the sedimentary record occurs at about 22 mbsf. The hiatus is estimated to range from 4.7 to $5.2 \mathrm{Ma}$ and to be a widespread hiatus in the deep sea (Keller and Barron, 1987). Below the hiatus the presence of Miocene microfossils indicates that the interval from 22 to 24 mbsf corresponds to magnetic polarity subchron C3AN-2. The diatom, radiolarian, and foraminiferal stratigraphy (Baldauf and Barron, this volume; Lazarus, in press; Schröder-Adams, this volume) indicates that a second hiatus occurs at 24 mbsf corresponding to 6.0 and $8.9 \mathrm{Ma}$. This hiatus is the boundary between lithologic Unit I and Unit II, and is also widespread in the deep sea (Keller and Barron, 1987).

The interval from 24 to 53 mbsf has been assigned to Chron 5 based upon diatoms, radiolarian, and calcareous nannofossil zonations (8.9-11.2 Ma, Barron et al., this volume). At 53 mbsf, a discontinuity was identified by a lithologic color change, a 10-fold increase in magnetic susceptibility (Dorn, this volume), and carbon isotope changes (Woodruff and Chamber, this volume). Between 41 and 83 mbsf several subchrons ( $5 \mathrm{~N}-2$ through 5E) within Chron 5 are identified.

The interval from 83 to 97 mbsf was been assigned to Chron $6 \mathrm{~N}$ with subchron $6 \mathrm{AN}-1$ identified above a discontinuity at about $97 \mathrm{~m}$. The discontinuity was recognized on the basis of diatom biostratigraphy (Baldauf and Barron, this volume), strontium isotope stratigraphy (Barrera et al, this volume), and the reversal stratigraphy.

The interval from 97 to 99 mbsf is assigned an age of 24-24.5 Ma based upon strontium isotope studies (Barrera et al, this volume) and overlapping ranges of nannofossil and diatom species (Barron et al., this volume). This interval overlies a discontinuity and is assigned to magnetic subchron $6 \mathrm{CN}-3$, on the basis of the magnetic reversal sequence at roughly $24.5-26.3 \mathrm{Ma}$ (Fig. 9).

The interval from 99 to about $118 \mathrm{mbsf}$ is assigned an age of upper Oligocene (roughly 26.3-28.4 Ma) on the basis of silicoflagellates (Bukry, this volume). The magnetic reversal sequence is correlated to seafloor magnetic anomalies $7 \mathrm{~A}, 8$, and 9. A discontinuity is present at about 118 mbsf based upon the strontium isotope results (Barrera et al., this volume) and oxygen isotope studies (Barrera and Huber, this volume). Fossil evidence (Baldauf and Barron, this volume; Wei and Thierstein, this volume; Huber, this volume) suggests this hiatus corresponds to a widespread Paleogene deep-sea hiatus (Keller et al., 1987). It is suggested that the middle Oligocene interval between 28.5 and $32 \mathrm{Ma}$ is either very compressed or missing (Barron et al., this volume).

Between about 118 and 162 mbsf, magnetic anomalies 11, 12,13 , and 15 are identified based upon age constraints provided by diatoms, radiolarians, calcareous nannofossils, and planktonic foraminifers (Barron et al, this volume). From roughly 162 to 176 mbsf, magnetic subchrons $16 \mathrm{~N}-1,16 \mathrm{~N}-2$, and $16 \mathrm{~N}-3$ are correlated, using biostratigraphic constraints provided by calcareous nannofossils and planktonic foraminifers.

\section{CONCLUSIONS}

Paleomagnetic studies of samples from Hole 744 indicate that the majority of the samples studied are characterized by very stable single-component magnetization, which record high paleolatitudes and frequent reversals. Isolated samples characterized by multiple components of magnetization were observed. These probably result from downhole contamination and Mnoxide mobilization. The samples displaying multicomponent magnetization have been omitted from the stratigraphic compilation. Using the data derived from the stably magnetized samples, a sequence of 45 polarity changes was observed. Age constraints based upon microfossils and isotopic studies were then used to place age constraints on the reversal sequence. The paleomagnetic, biostratigraphic, and isotope studies have been combined to establish a correlation between the reversal sequence in this hole and the geomagnetic time scale in order to provide a chronostratigraphic framework for sediments drilled at ODP Hole 744B. The studies indicate that a sequence of Quaternary to Eocene age has been recovered. Correlation of the reversal sequence to the geomagnetic time scale without these age constraints would have been a more difficult task since the stratigraphic sequence was substantially undersampled as a result of sampling restrictions. A correlation is suggested between the observed reversal sequence and seafloor anomalies 116. The results of these studies are a good example of the utility of combined biostratigraphic and paleomagnetic studies in establishing age constraints for deep-sea sediments.

\section{ACKNOWLEDGMENTS}

The shore-based cryogenic magnetometer measurements for this site required 4 continuous weeks of measurement by three individuals at the University of Hawaii. We am very grateful to James Lau and Professor Ma Xingua for all the days of hard work they contributed to this project. In addition, we wish to thank Dr. Barry Lienert for his very hard work in repairing the alternating demagnetization equipment, which required several days of repairs during a critical time when he was preparing for a site review. We also wish to thank Dr. Charles Helsley for spending days of his time making the Shibuya data recording system work and then assisting in the monumental data processing required to generate the stratigraphic plots used for this report. The software used for plotting is that of H. Shibuya, which was developed on Leg 124. We thank the reviewers for their comments and suggestions. This is Hawaii Institute of Geophysics Contribution 2302.

\section{REFERENCES}

Barron, J., Larsen, B., et al., 1989. Proc. ODP, Init. Repts., 119: College Station, TX (Ocean Drilling Program).

Berggren, W. A., Kent, D. V., Flynn, J. J., and Van Couvering, J. A., 1985. Cenozoic geochronology. Geol. Soc. Am. Bull., 96:14071418.

Keller, G., and Barron, J. A., 1987. Paleodepth distribution of Neogene deep-sea hiatuses. Paleoceanography, 2:697-713.

Keller, G., Herbert, T., Dorsey, R., D'Hondt, S., Johnsson, M., and Chi, W. R., 1987. Global distribution of late Paleogene hiatuses. Geology, 14:199-203.

Lazarus, D., in press. Antarctic Neogene radiolarians from the Kerguelen Plateau, ODP Legs 119 and 120. In Schlich, R., Wise, S. W., Jr., et al., Proc. ODP, Sci. Results, 120: College Station, TX (Ocean Drilling Program).

Malcolm, S. J., and Stanley, S. O., 1982. The sediment environment. In Nedwell, D. B., and Brown, C. M. (Eds.), Sediment Microbiology: London (Academic Press), 1-14.

Date of initial receipt: 8 December 1989

Date of acceptance: 16 July 1990

Ms 119B-147 
APPENDIX

\begin{tabular}{|c|c|c|c|c|c|c|c|c|}
\hline Core, section & $\begin{array}{c}\text { Interval } \\
(\mathrm{cm})\end{array}$ & $\begin{array}{l}\text { Depth } \\
\text { (mbsf) }\end{array}$ & S.T. & Demag level & Decl. & Incl. & Intensity & Polarity \\
\hline \multicolumn{9}{|l|}{$119-744 \mathrm{~A}-$} \\
\hline $1 \mathrm{H}-1$ & $130.0-132.0$ & 1.30 & MAG & $00 \mathrm{~A}$ & 89.4 & +61.7 & 2.2500 & $\mathbf{R}$ \\
\hline $1 \mathrm{H}-1$ & $130.0-132.0$ & 1.30 & MAG & $15 \mathrm{~A}$ & 109.0 & +60.1 & 1.8292 & $\mathbf{R}$ \\
\hline $1 \mathrm{H}-1$ & $130.0-132.0$ & 1.30 & MAG & $22 \mathrm{~A}$ & 88.0 & +66.6 & 1.4592 & $\mathbf{R}$ \\
\hline $1 \mathrm{H}-1$ & $130.0-132.0$ & 1.30 & MAG & $30 \mathrm{~A}$ & 91.9 & +57.3 & 1.2661 & $\mathbf{R}$ \\
\hline $1 \mathrm{H}-2$ & $42.0-44.0$ & 1.92 & MAG & $00 \mathrm{~A}$ & 86.5 & +3.6 & 8.8400 & $\mathbf{R}$ \\
\hline $1 \mathrm{H}-2$ & $42.0-44.0$ & 1.92 & MAG & $15 \mathrm{~A}$ & 49.3 & +25.2 & 6.5676 & $\mathbf{R}$ \\
\hline $1 \mathrm{H}-2$ & $42.0-44.0$ & 1.92 & MAG & $22 \mathrm{~A}$ & 49.7 & +20.2 & 5.3891 & $\mathbf{R}$ \\
\hline $1 \mathrm{H}-2$ & $42.0-44.0$ & 1.92 & MAG & $30 \mathrm{~A}$ & 48.5 & +18.9 & 4.4094 & $\mathbf{R}$ \\
\hline $1 \mathrm{H}-2$ & $103.0-105.0$ & 2.53 & MAG & $00 \mathrm{~A}$ & 93.6 & +2.4 & 16.9000 & $\mathbf{R}$ \\
\hline $1 \mathrm{H}-2$ & $103.0-105.0$ & 2.53 & MAG & $15 \mathrm{~A}$ & 57.0 & +45.7 & 7.8505 & $\mathbf{R}$ \\
\hline $1 \mathrm{H}-2$ & $103.0-105.0$ & 2.53 & MAG & $22 \mathrm{~A}$ & 55.3 & +56.2 & 5.3051 & $\mathbf{R}$ \\
\hline $1 \mathrm{H}-2$ & $103.0-105.0$ & 2.53 & MAG & $30 \mathrm{~A}$ & 87.5 & +56.7 & 3.0903 & $\mathbf{R}$ \\
\hline $1 \mathrm{H}-5$ & $120.0-122.0$ & 3.81 & MAG & $15 \mathrm{~A}$ & 188.8 & -5.6 & 4.2453 & A \\
\hline $1 \mathrm{H}-5$ & $120.0-122.0$ & 3.81 & MAG & $22 \mathrm{~A}$ & 187.1 & -2.2 & 3.0990 & A \\
\hline $1 \mathrm{H}-5$ & $120.0-122.0$ & 3.81 & MAG & $30 \mathrm{~A}$ & 192.8 & +7.6 & 2.7435 & A \\
\hline $1 \mathrm{H}-6$ & $120.0-122.0$ & 3.81 & MAG & $15 \mathrm{~A}$ & 205.4 & +34.8 & 10.5907 & A \\
\hline $1 \mathrm{H}-6$ & $120.0-122.0$ & 3.81 & MAG & $30 \mathrm{~A}$ & 202.6 & +35.5 & 7.6376 & A \\
\hline $2 \mathrm{H}-1$ & $90.0-92.0$ & 5.10 & MAG & $00 \mathrm{~A}$ & 159.3 & +87.7 & 17.0000 & $\mathbf{R}$ \\
\hline $2 \mathrm{H}-1$ & $90.0-92.0$ & 5.10 & MAG & $30 \mathrm{~A}$ & 133.7 & +58.8 & 8.2963 & $\mathbf{R}$ \\
\hline $2 \mathrm{H}-2$ & $90.0-92.0$ & 6.60 & MAG & $15 \mathrm{~A}$ & 46.4 & +69.2 & 16.2691 & $\mathbf{R}$ \\
\hline $2 \mathrm{H}-2$ & $90.0-92.0$ & 6.60 & MAG & $22 \mathrm{~A}$ & 55.3 & +70.3 & 12.6615 & $\mathbf{R}$ \\
\hline $2 \mathrm{H}-2$ & $90.0-92.0$ & 6.60 & MAG & $30 \mathrm{~A}$ & 49.2 & +63.4 & 10.0830 & $\mathbf{R}$ \\
\hline $2 \mathrm{H}-3$ & $41.0-43.0$ & 7.61 & MAG & $00 \mathrm{~A}$ & 92.7 & +77.0 & 8.2300 & $\mathbf{R}$ \\
\hline $2 \mathrm{H}-3$ & $41.0-43.0$ & 7.61 & MAG & $15 \mathrm{~A}$ & 240.5 & +69.6 & 5.9139 & $\mathbf{R}$ \\
\hline $2 \mathrm{H}-3$ & $41.0-43.0$ & 7.61 & MAG & $22 \mathrm{~A}$ & 250.1 & +61.8 & 5.1059 & $\mathbf{R}$ \\
\hline $2 \mathrm{H}-3$ & $41.0-43.0$ & 7.61 & MAG & $30 \mathrm{~A}$ & 246.8 & +62.6 & 4.8109 & $\mathbf{R}$ \\
\hline $2 \mathrm{H}-3$ & $111.0-113.0$ & 8.31 & MAG & $00 \mathrm{~A}$ & 268.9 & +56.7 & 33.3000 & $\mathbf{R}$ \\
\hline $2 \mathrm{H}-3$ & $111.0-113.0$ & 8.31 & MAG & $15 \mathrm{~A}$ & 269.6 & +72.4 & 15.7812 & $\mathbf{R}$ \\
\hline $2 \mathrm{H}-3$ & $111.0-113.0$ & 8.31 & MAG & $22 \mathrm{~A}$ & 263.6 & +71.6 & 16.0446 & $\mathbf{R}$ \\
\hline $2 \mathrm{H}-3$ & $111.0-113.0$ & 8.31 & MAG & $30 \mathrm{~A}$ & 327.9 & +67.6 & 11.2865 & $\mathbf{R}$ \\
\hline $2 \mathrm{H}-4$ & $40.0-42.0$ & 9.10 & MAG & $00 \mathrm{~A}$ & 258.8 & +72.1 & 27.1000 & $\mathbf{R}$ \\
\hline $2 \mathrm{H}-4$ & $40.0-42.0$ & 9.10 & MAG & $15 \mathrm{~A}$ & 183.9 & +43.7 & 16.2966 & $\mathbf{R}$ \\
\hline $2 \mathrm{H}-4$ & $40.0-42.0$ & 9.10 & MAG & $22 \mathrm{~A}$ & 179.9 & +41.5 & 12.4351 & $\mathbf{R}$ \\
\hline $2 \mathrm{H}-4$ & $40.0-42.0$ & 9.10 & MAG & $30 \mathrm{~A}$ & 176.2 & +32.1 & 11.1213 & $\mathbf{R}$ \\
\hline $2 \mathrm{H}-4$ & $105.0-107.0$ & 9.75 & MAG & $00 \mathrm{~A}$ & 257.2 & +82.5 & 52.5000 & $\mathbf{R}$ \\
\hline $2 \mathrm{H}-4$ & $105.0-107.0$ & 9.75 & MAG & $15 \mathrm{~A}$ & 179.7 & +72.6 & 37.9976 & $\mathbf{R}$ \\
\hline $2 \mathrm{H}-4$ & $105.0-107.0$ & 9.75 & MAG & $22 \mathrm{~A}$ & 184.4 & +74.4 & 28.0313 & $\mathbf{R}$ \\
\hline $2 \mathrm{H}-4$ & $105.0-107.0$ & 9.75 & MAG & $30 \mathrm{~A}$ & 177.8 & +73.1 & 22.1676 & $\mathbf{R}$ \\
\hline $3 \mathrm{H}-3$ & $62.0-64.0$ & 17.32 & MAG & $00 \mathrm{~A}$ & 86.3 & +76.2 & 54.4000 & $\mathbf{R}$ \\
\hline $3 \mathrm{H}-4$ & $12.0-14.0$ & 18.32 & MAG & $00 \mathrm{~A}$ & 14.8 & -50.2 & 4.0431 & A \\
\hline $3 \mathrm{H}-4$ & $12.0-14.0$ & 18.32 & MAG & $00 \mathrm{~A}$ & 283.1 & +83.7 & 46.6000 & A \\
\hline $3 \mathrm{H}-4$ & $62.0-64.0$ & 18.82 & MAG & $00 \mathrm{~A}$ & 305.9 & +85.9 & 23.3000 & $\mathbf{R}$ \\
\hline $3 \mathrm{H}-4$ & $62.0-64.0$ & 18.82 & MAG & $15 \mathrm{~A}$ & 54.1 & +79.2 & 5.6539 & $\mathbf{R}$ \\
\hline $3 \mathrm{H}-4$ & $62.0-64.0$ & 18.82 & MAG & $30 \mathrm{~A}$ & 52.8 & +71.3 & 5.2023 & $\mathbf{R}$ \\
\hline $3 \mathrm{H}-4$ & $136.0-138.0$ & 19.56 & MAG & $00 \mathrm{~A}$ & 168.2 & +86.6 & 48.6000 & A \\
\hline $3 \mathrm{H}-4$ & $136.0-138.0$ & 19.56 & MAG & $15 \mathrm{~A}$ & 168.3 & +39.3 & 22.2763 & A \\
\hline $3 \mathrm{H}-4$ & $136.0-138.0$ & 19.56 & MAG & $30 \mathrm{~A}$ & 168.7 & +39.1 & 8.8148 & A \\
\hline $4 \mathrm{H}-1$ & $50.0-52.0$ & 23.70 & MAG & $00 \mathrm{~A}$ & 250.3 & -17.8 & 3.7400 & A \\
\hline $4 \mathrm{H}-1$ & $50.0-52.0$ & 23.70 & MAG & $15 \mathrm{~A}$ & 183.0 & -15.1 & 4.5292 & A \\
\hline $4 \mathrm{H}-1$ & $50.0-52.0$ & 23.70 & MAG & $22 \mathrm{~A}$ & 207.7 & -8.0 & 3.0010 & A \\
\hline $4 \mathrm{H}-1$ & $50.0-52.0$ & 23.70 & MAG & $30 \mathrm{~A}$ & 202.4 & +25.3 & 1.3625 & A \\
\hline $4 \mathrm{H}-2$ & $110.0-112.0$ & 25.80 & MAG & $00 \mathrm{~A}$ & 299.8 & +43.6 & 1.1274 & $\mathbf{R}$ \\
\hline $4 \mathrm{H}-2$ & $110.0-112.0$ & 25.80 & MAG & $05 \mathrm{~A}$ & 227.8 & +35.5 & 4.1200 & $\mathbf{R}$ \\
\hline $4 \mathrm{H}-2$ & $110.0-112.0$ & 25.80 & MAG & $10 \mathrm{~A}$ & 224.2 & +35.4 & 3.7400 & $\mathbf{R}$ \\
\hline $4 \mathrm{H}-2$ & $110.0-112.0$ & 24.30 & MAG & $15 \mathrm{~A}$ & 172.4 & +41.7 & 6.6631 & $\mathbf{R}$ \\
\hline $4 \mathrm{H}-2$ & $110.0-112.0$ & 25.80 & MAG & $30 \mathrm{~A}$ & 238.5 & +40.9 & 1.1800 & $\mathbf{R}$ \\
\hline $4 \mathrm{H}-2$ & $110.0-112.0$ & 25.80 & MAG & $40 \mathrm{~A}$ & 222.6 & -59.2 & 0.6080 & $\mathbf{R}$ \\
\hline $4 \mathrm{H}-2$ & $110.0-112.0$ & 25.80 & MAG & $50 \mathrm{~A}$ & 155.0 & -3.4 & 5.9700 & $\mathbf{R}$ \\
\hline $4 \mathrm{H}-3$ & $50.0-52.0$ & 26.70 & MAG & $00 \mathrm{~A}$ & 262.1 & +65.4 & 3.9600 & $\mathbf{R}$ \\
\hline $4 \mathrm{H}-3$ & $50.0-52.0$ & 25.20 & MAG & $15 \mathrm{~A}$ & 188.7 & +74.8 & 4.3698 & $\mathbf{R}$ \\
\hline $4 \mathrm{H}-3$ & $50.0-52.0$ & 26.70 & MAG & $22 \mathrm{~A}$ & 154.3 & +64.2 & 1.9918 & $\mathbf{R}$ \\
\hline $4 \mathrm{H}-3$ & $50.0-52.0$ & 26.70 & MAG & $30 \mathrm{~A}$ & 141.1 & +57.6 & 1.3945 & $\mathbf{R}$ \\
\hline $4 \mathrm{H}-3$ & $110.0-112.0$ & 27.30 & MAG & $00 \mathrm{~A}$ & 254.9 & +3.6 & 1.8200 & $\mathbf{R}$ \\
\hline $4 \mathrm{H}-3$ & $110.0-112.0$ & 27.30 & MAG & $15 \mathrm{~A}$ & 148.8 & +26.3 & 3.5518 & $\mathbf{R}$ \\
\hline $4 \mathrm{H}-3$ & $110.0-112.0$ & 27.30 & MAG & $22 \mathrm{~A}$ & 158.8 & +22.0 & 2.0783 & $\mathbf{R}$ \\
\hline $4 \mathrm{H}-3$ & $110.0-112.0$ & 27.30 & MAG & $30 \mathrm{~A}$ & 160.1 & +13.6 & 1.6845 & $\mathbf{R}$ \\
\hline $4 \mathrm{H}-4$ & $50.0-52.0$ & 28.20 & MAG & $00 \mathrm{~A}$ & 268.1 & -0.8 & 3.9800 & A \\
\hline $4 \mathrm{H}-4$ & $50.0-52.0$ & 28.20 & MAG & $15 \mathrm{~A}$ & 175.0 & +8.5 & 0.8403 & A \\
\hline $4 \mathrm{H}-4$ & $50.0-52.0$ & 28.20 & MAG & $30 \mathrm{~A}$ & 155.8 & -22.3 & 0.4059 & A \\
\hline $5 \mathrm{H}-1$ & $110.0-112.0$ & 33.80 & MAG & $00 \mathrm{~A}$ & 263.2 & +10.7 & 8.1000 & A \\
\hline $5 \mathrm{H}-1$ & $110.0-112.0$ & 33.80 & MAG & $15 \mathrm{~A}$ & 215.2 & +19.8 & 3.6940 & A \\
\hline $5 \mathrm{H}-1$ & $110.0-112.0$ & 33.80 & MAG & $30 \mathrm{~A}$ & 206.3 & +7.6 & 1.8106 & A \\
\hline $5 \mathrm{H}-2$ & $50.0-52.0$ & 34.70 & MAG & $00 \mathrm{~A}$ & 262.3 & -10.6 & 6.7000 & A \\
\hline $5 \mathrm{H}-2$ & $50.0-52.0$ & 34.70 & MAG & $15 \mathrm{~A}$ & 205.9 & -5.9 & 3.2943 & A \\
\hline $5 \mathrm{H}-2$ & $50.0-52.0$ & 34.70 & MAG & $30 \mathrm{~A}$ & 207.5 & -21.1 & 1.7733 & A \\
\hline $5 \mathrm{H}-2$ & $110.0-112.0$ & 35.30 & MAG & $00 \mathrm{~A}$ & 259.7 & -0.3 & 7.4500 & $\mathbf{N}$ \\
\hline
\end{tabular}


Appendix (continued).

\begin{tabular}{|c|c|c|c|c|c|c|c|c|}
\hline Core, section & $\begin{array}{c}\text { Interval } \\
(\mathrm{cm})\end{array}$ & $\begin{array}{l}\text { Depth } \\
\text { (mbsf) }\end{array}$ & S.T. & Demag level & Decl. & Incl. & Intensity & Polarity \\
\hline \multicolumn{9}{|l|}{ 119-744A-(Cont.) } \\
\hline $5 \mathrm{H}-2$ & $110.0-112.0$ & 35.30 & MAG & $15 \mathrm{~A}$ & 194.2 & -22.0 & 5.0493 & $\mathrm{~N}$ \\
\hline $5 \mathrm{H}-2$ & $110.0-112.0$ & 35.30 & MAG & $22 \mathrm{~A}$ & 195.2 & -32.1 & 2.9817 & $\mathrm{~N}$ \\
\hline $5 \mathrm{H}-2$ & $110.0-112.0$ & 35.30 & MAG & $30 \mathrm{~A}$ & 192.4 & -37.8 & 2.5653 & $\mathrm{~N}$ \\
\hline $5 \mathrm{H}-3$ & $50.0-52.0$ & 36.20 & MAG & $00 \mathrm{~A}$ & 259.8 & +45.3 & 8.2400 & $\mathbf{R}$ \\
\hline $5 \mathrm{H}-3$ & $50.0-52.0$ & 36.20 & MAG & $15 \mathrm{~A}$ & 178.6 & +22.4 & 5.1538 & $\mathbf{R}$ \\
\hline $5 \mathrm{H}-3$ & $50.0-52.0$ & 36.20 & MAG & $22 \mathrm{~A}$ & 182.1 & +18.7 & 2.9656 & $\mathbf{R}$ \\
\hline $5 \mathrm{H}-3$ & $50.0-52.0$ & 36.20 & MAG & $30 \mathrm{~A}$ & 189.2 & +3.4 & 2.1609 & $\mathbf{R}$ \\
\hline $5 \mathrm{H}-3$ & $110.0-112.0$ & 36.80 & MAG & $00 \mathrm{~A}$ & 261.7 & +46.0 & 6.8900 & $\mathbf{R}$ \\
\hline $5 \mathrm{H}-3$ & $110.0-112.0$ & 36.80 & MAG & $15 \mathrm{~A}$ & 189.6 & +49.0 & 4.7988 & $\mathbf{R}$ \\
\hline $5 \mathrm{H}-3$ & $110.0-112.0$ & 36.80 & MAG & $22 \mathrm{~A}$ & 190.2 & +46.6 & 3.5336 & $\mathbf{R}$ \\
\hline $5 \mathrm{H}-3$ & $110.0-112.0$ & 36.80 & MAG & $30 \mathrm{~A}$ & 190.3 & +47.4 & 2.2194 & $\mathbf{R}$ \\
\hline $5 \mathrm{H}-3$ & $130.0-132.0$ & 37.00 & MAG & $00 \mathrm{~A}$ & 89.9 & -45.5 & 7.3700 & $\mathrm{~N}$ \\
\hline $5 \mathrm{H}-3$ & $130.0-132.0$ & 37.00 & MAG & $15 \mathrm{~A}$ & 90.7 & -21.5 & 2.6279 & $\mathbf{N}$ \\
\hline $5 \mathrm{H}-3$ & $130.0-132.0$ & 37.00 & MAG & $22 \mathrm{~A}$ & 85.8 & -24.7 & 1.5561 & $\mathrm{~N}$ \\
\hline $5 \mathrm{H}-3$ & $130.0-132.0$ & 37.00 & MAG & $30 \mathrm{~A}$ & 85.1 & -21.0 & 1.3134 & $\mathrm{~N}$ \\
\hline $5 \mathrm{H}-4$ & $28.0-30.0$ & 37.48 & MAG & $00 \mathrm{~A}$ & 263.9 & +36.4 & 6.9300 & $\mathbf{R}$ \\
\hline $5 \mathrm{H}-4$ & $28.0-30.0$ & 37.48 & MAG & $22 \mathrm{~A}$ & 218.3 & +48.5 & 2.4896 & $\mathbf{R}$ \\
\hline $5 \mathrm{H}-4$ & $28.0-30.0$ & 37.48 & MAG & $30 \mathrm{~A}$ & 204.0 & +44.1 & 1.5129 & $\mathbf{R}$ \\
\hline $5 \mathrm{H}-4$ & $28.0-30.0$ & 37.48 & MAG & $15 \mathrm{~A}$ & 216.0 & +50.3 & 3.7801 & $\mathbf{R}$ \\
\hline $5 \mathrm{H}-5$ & $130.0-132.0$ & 40.00 & MAG & $00 \mathrm{~A}$ & 91.6 & -51.9 & 18.2000 & $N$ \\
\hline $5 \mathrm{H}-5$ & $130.0-132.0$ & 40.00 & MAG & $15 \mathrm{~A}$ & 111.9 & -47.7 & 5.0472 & $\mathbf{N}$ \\
\hline $5 \mathrm{H}-5$ & $130.0-132.0$ & 40.00 & MAG & $22 \mathrm{~A}$ & 117.7 & -52.6 & 3.6264 & $\mathbf{N}$ \\
\hline $5 \mathrm{H}-5$ & $130.0-132.0$ & 40.00 & MAG & $30 \mathrm{~A}$ & 113.4 & -62.0 & 2.5463 & $\mathrm{~N}$ \\
\hline $6 \mathrm{H}-1$ & $126.0-128.0$ & 43.46 & MAG & $00 \mathrm{~A}$ & 260.4 & +36.3 & 2.3800 & $\mathbf{R}$ \\
\hline $6 \mathrm{H}-1$ & $126.0-128.0$ & 43.46 & MAG & $15 \mathrm{~A}$ & 201.9 & +35.1 & 1.9705 & $\mathbf{R}$ \\
\hline $6 \mathrm{H}-1$ & $126.0-128.0$ & 43.46 & MAG & $22 \mathrm{~A}$ & 199.0 & +43.5 & 1.3095 & $\mathbf{R}$ \\
\hline $6 \mathrm{H}-1$ & $126.0-128.0$ & 43.46 & MAG & $30 \mathrm{~A}$ & 224.7 & +31.3 & 1.0326 & $\mathbf{R}$ \\
\hline $6 \mathrm{H}-2$ & $44.0-46.0$ & 44.14 & MAG & $00 \mathrm{~A}$ & 261.0 & +71.4 & 4.6500 & $\mathbf{R}$ \\
\hline $6 \mathrm{H}-2$ & $44.0-46.0$ & 44.14 & MAG & $15 \mathrm{~A}$ & 171.9 & +80.1 & 3.3754 & $\mathbf{R}$ \\
\hline $6 \mathrm{H}-2$ & $44.0-46.0$ & 44.14 & MAG & $22 \mathrm{~A}$ & 173.0 & +78.0 & 2.5381 & $\mathrm{R}$ \\
\hline $6 \mathrm{H}-2$ & $44.0-46.0$ & 44.14 & MAG & $30 \mathrm{~A}$ & 183.4 & +76.3 & 1.6269 & $\mathbf{R}$ \\
\hline $6 \mathrm{H}-4$ & $67.0-69.0$ & 47.37 & MAG & $00 \mathrm{~A}$ & 215.2 & +88.3 & 9.3800 & $\mathbf{R}$ \\
\hline $6 \mathrm{H}-4$ & $67.0-69.0$ & 47.37 & MAG & $15 \mathrm{~A}$ & 110.1 & +74.1 & 7.8781 & $\mathbf{R}$ \\
\hline $6 \mathrm{H}-4$ & $67.0-69.0$ & 47.37 & MAG & $22 \mathrm{~A}$ & 123.2 & +73.4 & 5.9414 & $\mathbf{R}$ \\
\hline $6 \mathrm{H}-4$ & $67.0-69.0$ & 47.37 & MAG & $30 \mathrm{~A}$ & 112.3 & +71.1 & 3.9785 & $\mathbf{R}$ \\
\hline $6 \mathrm{H}-6$ & $80.0-82.0$ & 47.50 & MAG & $00 \mathrm{~A}$ & 89.0 & +15.1 & 2.3300 & $\mathbf{R}$ \\
\hline $6 \mathrm{H}-6$ & $80.0-82.0$ & 47.50 & MAG & $15 \mathrm{~A}$ & 98.9 & +52.0 & 2.1062 & $\mathbf{R}$ \\
\hline $6 \mathrm{H}-6$ & $80.0-82.0$ & 47.50 & MAG & $22 \mathrm{~A}$ & 98.1 & +62.8 & 1.2862 & $\mathbf{R}$ \\
\hline $6 \mathrm{H}-6$ & $80.0-82.0$ & 47.50 & MAG & $30 \mathrm{~A}$ & 95.4 & +60.2 & 0.9933 & $\mathbf{R}$ \\
\hline $6 \mathrm{H}-6$ & $124.0-126$ & 47.96 & MAG & $00 \mathrm{~A}$ & 88.0 & -9.6 & 5.6800 & $\mathbf{R}$ \\
\hline $6 \mathrm{H}-6$ & $124.0-126.0$ & 47.96 & MAG & $15 \mathrm{~A}$ & 88.3 & +16.7 & 3.1971 & $\mathbf{R}$ \\
\hline $6 \mathrm{H}-6$ & $124.0-126.0$ & 47.96 & MAG & $22 \mathrm{~A}$ & 89.3 & +18.8 & 1.8919 & $\mathbf{R}$ \\
\hline $6 \mathrm{H}-6$ & $124.0-126.0$ & 47.96 & MAG & $30 \mathrm{~A}$ & 92.0 & +20.2 & 1.4584 & $\mathbf{R}$ \\
\hline $7 \mathrm{H}-1$ & $79.0-81.0$ & 52.47 & MAG & $00 \mathrm{~A}$ & 263.7 & +50.5 & 17.3000 & $\mathbf{R}$ \\
\hline $7 \mathrm{H}-1$ & $79.0-81.0$ & 52.47 & MAG & $15 \mathrm{~A}$ & 195.5 & +51.2 & 5.9787 & $\mathbf{R}$ \\
\hline $7 \mathrm{H}-1$ & $79.0-81.0$ & 52.47 & MAG & $22 \mathrm{~A}$ & 188.8 & +55.4 & 3.8817 & $\mathbf{R}$ \\
\hline $7 \mathrm{H}-1$ & $79.0-81.0$ & 52.47 & MAG & $30 \mathrm{~A}$ & 189.9 & +55.5 & 2.6897 & $\mathbf{R}$ \\
\hline $7 \mathrm{H}-1$ & $113.0-115.0$ & 52.83 & MAG & $00 \mathrm{~A}$ & 266.5 & +1.8 & 6.3400 & $\mathbf{R}$ \\
\hline $7 \mathrm{H}-1$ & $113.0-115.0$ & 52.83 & MAG & $15 \mathrm{~A}$ & 216.5 & +54.5 & 3.1117 & $\mathbf{R}$ \\
\hline $7 \mathrm{H}-1$ & $113.0-115.0$ & 52.83 & MAG & $22 \mathrm{~A}$ & 217.7 & +50.3 & 2.3614 & $\mathbf{R}$ \\
\hline $7 \mathrm{H}-1$ & $113.0-115.0$ & 52.83 & MAG & $30 \mathrm{~A}$ & 212.1 & +58.6 & 1.3906 & $\mathbf{R}$ \\
\hline $7 \mathrm{H}-1$ & $145.0-147.0$ & 53.15 & MAG & $00 \mathrm{~A}$ & 262.0 & +18.2 & 3.0100 & $\mathbf{R}$ \\
\hline $7 \mathrm{H}-1$ & $145.0-147.0$ & 53.15 & MAG & $15 \mathrm{~A}$ & 178.3 & +55.9 & 2.0456 & $\mathbf{R}$ \\
\hline $7 \mathrm{H}-1$ & $145.0-147.0$ & 53.15 & MAG & $22 \mathrm{~A}$ & 175.3 & +56.3 & 1.7883 & $\mathbf{R}$ \\
\hline $7 \mathrm{H}-1$ & $145.0-147.0$ & 53.15 & MAG & $30 \mathrm{~A}$ & 171.1 & +72.9 & 0.9346 & $\mathbf{R}$ \\
\hline $7 \mathrm{H}-2$ & $22.0-24.0$ & 53.42 & MAG & $00 \mathrm{~A}$ & 260.6 & +45.8 & 4.1400 & $\mathbf{R}$ \\
\hline $7 \mathrm{H}-2$ & $22.0-24.0$ & 53.42 & MAG & $15 \mathrm{~A}$ & 185.7 & +65.6 & 2.5875 & $\mathbf{R}$ \\
\hline $7 \mathrm{H}-2$ & $22.0-24.0$ & 53.42 & MAG & $22 \mathrm{~A}$ & 166.4 & +66.1 & 1.8623 & $\mathbf{R}$ \\
\hline $7 \mathrm{H}-2$ & $22.0-24.0$ & 53.42 & MAG & $30 \mathrm{~A}$ & 206.6 & +71.9 & 1.0317 & $\mathrm{R}$ \\
\hline $7 \mathrm{H}-2$ & $64.0-66.0$ & 53.84 & MAG & $00 \mathrm{~A}$ & 266.4 & +39.3 & 7.1900 & $\mathbf{R}$ \\
\hline $7 \mathrm{H}-2$ & $64.0-66.0$ & 53.84 & MAG & $15 \mathrm{~A}$ & 217.1 & +70.2 & 2.7202 & $\mathbf{R}$ \\
\hline $7 \mathrm{H}-2$ & $64.0-66.0$ & 53.84 & MAG & $22 \mathrm{~A}$ & 224.5 & +71.9 & 1.9563 & $\mathbf{R}$ \\
\hline $7 \mathrm{H}-2$ & $64.0-66.0$ & 53.84 & MAG & $30 \mathrm{~A}$ & 232.2 & +73.9 & 1.3209 & $\mathbf{R}$ \\
\hline $7 \mathrm{H}-2$ & $144.0-146.0$ & 54.64 & MAG & $00 \mathrm{~A}$ & 263.7 & +22.1 & 3.6900 & $\mathbf{R}$ \\
\hline $7 \mathrm{H}-2$ & $144.0-146.0$ & 54.64 & MAG & $15 \mathrm{~A}$ & 175.0 & +16.4 & 0.9642 & $\mathbf{R}$ \\
\hline $7 \mathrm{H}-2$ & $144.0-146.0$ & 54.64 & MAG & $22 \mathrm{~A}$ & 187.2 & +24.4 & 0.8871 & $\mathbf{R}$ \\
\hline $7 \mathrm{H}-2$ & $144.0-146.0$ & 54.64 & MAG & $30 \mathrm{~A}$ & 206.3 & +9.0 & 0.4326 & $\mathbf{R}$ \\
\hline $7 \mathrm{H}-3$ & $21.0-23.0$ & 54.91 & MAG & $00 \mathrm{~A}$ & 265.1 & +35.9 & 4.1500 & $\mathbf{R}$ \\
\hline $7 \mathrm{H}-3$ & $21.0-23.0$ & 54.91 & MAG & $15 \mathrm{~A}$ & 174.2 & +50.1 & 1.2182 & $\mathbf{R}$ \\
\hline $7 \mathrm{H}-3$ & $21.0-23.0$ & 54.91 & MAG & $22 \mathrm{~A}$ & 138.5 & +53.5 & 0.9890 & $\mathrm{R}$ \\
\hline $7 \mathrm{H}-3$ & $21.0-23.0$ & 54.91 & MAG & $30 \mathrm{~A}$ & 209.7 & +45.2 & 0.7178 & $\mathbf{R}$ \\
\hline $7 \mathrm{H}-3$ & $68.0-70.0$ & 55.38 & MAG & $00 \mathrm{~A}$ & 265.0 & -56.9 & 5.6200 & $\mathbf{N}$ \\
\hline $7 \mathrm{H}-3$ & $68.0-70.0$ & 55.38 & MAG & $15 \mathrm{~A}$ & 112.8 & -69.6 & 3.4908 & $\mathbf{N}$ \\
\hline $7 \mathrm{H}-3$ & $68.0-70.0$ & 55.38 & MAG & $22 \mathrm{~A}$ & 102.9 & -76.5 & 2.3550 & $\mathrm{~N}$ \\
\hline $7 \mathrm{H}-3$ & $68.0-70.0$ & 55.38 & MAG & $30 \mathrm{~A}$ & 91.6 & -68.1 & 1.7415 & $\mathrm{~N}$ \\
\hline $7 \mathrm{H}-3$ & $124.0-126.0$ & 55.94 & MAG & $00 \mathrm{~A}$ & 262.8 & -52.3 & 5.5700 & $\mathrm{~N}$ \\
\hline
\end{tabular}


Appendix (continued).

\begin{tabular}{|c|c|c|c|c|c|c|c|c|}
\hline Core, section & $\begin{array}{c}\text { Interval } \\
(\mathrm{cm})\end{array}$ & $\begin{array}{l}\text { Depth } \\
\text { (mbsf) }\end{array}$ & S.T. & Demag level & Decl. & Incl. & Intensity & Polarity \\
\hline \multicolumn{9}{|l|}{ 119-744A-(Cont.) } \\
\hline $7 \mathrm{H}-3$ & $124.0-126.0$ & 55.94 & MAG & $15 \mathrm{~A}$ & 147.2 & -55.2 & 3.3605 & $\mathrm{~N}$ \\
\hline $7 \mathrm{H}-3$ & $124.0-126.0$ & 55.94 & MAG & $22 \mathrm{~A}$ & 149.0 & -68.0 & 2.2623 & $\mathrm{~N}$ \\
\hline $7 \mathrm{H}-3$ & $124.0-126.0$ & 55.94 & MAG & $30 \mathrm{~A}$ & 126.2 & -70.3 & 1.8537 & $\mathrm{~N}$ \\
\hline $7 \mathrm{H}-4$ & $13.0-15.0$ & 56.33 & MAG & $00 \mathrm{~A}$ & 243.7 & +40.1 & 1.1800 & A \\
\hline $7 \mathrm{H}-4$ & $13.0-15.0$ & 56.33 & MAG & $15 \mathrm{~A}$ & 160.9 & -37.8 & 2.2395 & A \\
\hline $7 \mathrm{H}-4$ & $13.0-15.0$ & 56.33 & MAG & $30 \mathrm{~A}$ & 160.7 & -39.8 & 1.1034 & A \\
\hline $7 \mathrm{H}-4$ & $64.0-66.0$ & 56.84 & MAG & $00 \mathrm{~A}$ & 262.9 & -23.8 & 4.4000 & $\mathbf{N}$ \\
\hline $7 \mathrm{H}-4$ & $64.0-66.0$ & 56.84 & MAG & $15 \mathrm{~A}$ & 155.1 & -45.7 & 1.7161 & $\mathrm{~N}$ \\
\hline $7 \mathrm{H}-4$ & $64.0-66.0$ & 56.84 & MAG & $22 \mathrm{~A}$ & 151.5 & -24.6 & 0.9456 & $\mathrm{~N}$ \\
\hline $7 \mathrm{H}-4$ & $64.0-66.0$ & 56.84 & MAG & $30 \mathrm{~A}$ & 139.5 & -39.8 & 0.7619 & $\mathrm{~N}$ \\
\hline $7 \mathrm{H}-4$ & $134.0-136.0$ & 57.54 & MAG & $00 \mathrm{~A}$ & 255.2 & -79.0 & 8.2600 & $\mathbf{N}$ \\
\hline $7 \mathrm{H}-4$ & $134.0-136.0$ & 57.54 & MAG & $15 \mathrm{~A}$ & 52.7 & -75.1 & 6.5127 & $\mathrm{~N}$ \\
\hline $7 \mathrm{H}-4$ & $134.0-136.0$ & 57.54 & MAG & $22 \mathrm{~A}$ & 155.5 & -76.9 & 5.1400 & $\mathrm{~N}$ \\
\hline $7 \mathrm{H}-4$ & $134.0-136.0$ & 57.54 & MAG & $30 \mathrm{~A}$ & 131.5 & -71.4 & 3.8258 & $\mathrm{~N}$ \\
\hline $7 \mathrm{H}-5$ & $7.0-9.0$ & 57.77 & MAG & $00 \mathrm{~A}$ & 262.6 & +71.2 & 7.2300 & $\mathbf{R}$ \\
\hline $7 \mathrm{H}-5$ & $7.0-9.0$ & 57.77 & MAG & $15 \mathrm{~A}$ & 213.5 & +73.8 & 4.7850 & $\mathbf{R}$ \\
\hline $7 \mathrm{H}-5$ & $7.0-9.0$ & 57.77 & MAG & $22 \mathrm{~A}$ & 213.6 & +75.8 & 3.2780 & $\mathbf{R}$ \\
\hline $7 \mathrm{H}-5$ & $7.0-9.0$ & 57.77 & MAG & $30 \mathrm{~A}$ & 227.7 & +76.7 & 2.4801 & $\mathbf{R}$ \\
\hline $7 \mathrm{H}-5$ & $68.0-70.0$ & 58.38 & MAG & $00 \mathrm{~A}$ & 266.7 & +68.7 & 12.4000 & $\mathbf{R}$ \\
\hline $7 \mathrm{H}-5$ & $68.0-70.0$ & 58.38 & MAG & $15 \mathrm{~A}$ & 265.5 & +75.2 & 8.6163 & $\mathbf{R}$ \\
\hline $7 \mathrm{H}-5$ & $68.0-70.0$ & 58.38 & MAG & $22 \mathrm{~A}$ & 270.3 & +75.3 & 6.2869 & $\mathbf{R}$ \\
\hline $7 \mathrm{H}-5$ & $68.0-70.0$ & 58.38 & MAG & $30 \mathrm{~A}$ & 275.3 & +75.3 & 4.5373 & $\mathbf{R}$ \\
\hline $7 \mathrm{H}-5$ & $95.0-97.0$ & 58.65 & MAG & $00 \mathrm{~A}$ & 264.8 & +63.3 & 9.5500 & $\mathbf{R}$ \\
\hline $7 \mathrm{H}-5$ & $95.0-97.0$ & 58.65 & MAG & $15 \mathrm{~A}$ & 243.6 & +63.0 & 6.2502 & $\mathbf{R}$ \\
\hline $7 \mathrm{H}-5$ & $95.0-97.0$ & 58.65 & MAG & $22 \mathrm{~A}$ & 240.9 & +67.5 & 4.6661 & $\mathbf{R}$ \\
\hline $7 \mathrm{H}-5$ & $95.0-97.0$ & 58.65 & MAG & $30 \mathrm{~A}$ & 229.8 & +70.4 & 3.4983 & $\mathbf{R}$ \\
\hline $7 \mathrm{H}-6$ & $6.0-8.0$ & 58.76 & MAG & $00 \mathrm{~A}$ & 263.2 & +63.5 & 6.1500 & $\mathbf{R}$ \\
\hline $7 \mathrm{H}-6$ & $6.0-8.0$ & 58.76 & MAG & $15 \mathrm{~A}$ & 222.7 & +68.4 & 3.4194 & $\mathbf{R}$ \\
\hline $7 \mathrm{H}-6$ & $6.0-8.0$ & 58.76 & MAG & $22 \mathrm{~A}$ & 212.5 & +66.5 & 2.4809 & $\mathbf{R}$ \\
\hline $7 \mathrm{H}-6$ & $6.0-8.0$ & 58.76 & MAG & $30 \mathrm{~A}$ & 205.1 & +72.6 & 1.8043 & $\mathbf{R}$ \\
\hline $7 \mathrm{H}-6$ & $54.0-56.0$ & 59.24 & MAG & $00 \mathrm{~A}$ & 255.3 & +59.1 & 2.8800 & A \\
\hline $7 \mathrm{H}-6$ & $54.0-56.0$ & 59.24 & MAG & $15 \mathrm{~A}$ & 159.2 & +33.8 & 1.9834 & A \\
\hline $7 \mathrm{H}-6$ & $54.0-56.0$ & 59.24 & MAG & $30 \mathrm{~A}$ & 151.1 & +7.9 & 0.7438 & A \\
\hline $8 \mathrm{H}-1$ & $25.0-27.0$ & 61.45 & MAG & $00 \mathrm{~A}$ & 267.0 & +49.4 & 16.4000 & $\mathbf{R}$ \\
\hline $8 \mathrm{H}-1$ & $25.0-27.0$ & 61.45 & MAG & $22 \mathrm{~A}$ & 234.0 & +58.3 & 7.5036 & $\mathbf{R}$ \\
\hline $8 \mathrm{H}-1$ & $25.0-27.0$ & 61.45 & MAG & $30 \mathrm{~A}$ & 231.0 & +62.4 & 5.1147 & $\mathbf{R}$ \\
\hline $8 \mathrm{H}-1$ & $141.0-143.0$ & 62.61 & MAG & $22 \mathrm{~A}$ & 68.0 & -79.1 & 8.7589 & $\mathrm{~N}$ \\
\hline $8 \mathrm{H}-1$ & $141.0-143.0$ & 62.61 & MAG & $30 \mathrm{~A}$ & 70.6 & -76.5 & 6.0680 & $\mathrm{~N}$ \\
\hline $8 \mathrm{H}-1$ & $145.0-147.0$ & 62.65 & MAG & $00 \mathrm{~A}$ & 83.9 & -78.1 & 23.1000 & $\mathrm{~N}$ \\
\hline $8 \mathrm{H}-1$ & $145.0-147.0$ & 62.65 & MAG & $15 \mathrm{~A}$ & 335.3 & -74.0 & 7.0982 & $\mathbf{N}$ \\
\hline $8 \mathrm{H}-1$ & $145.0-147.0$ & 62.65 & MAG & $22 \mathrm{~A}$ & 331.5 & -72.7 & 5.1605 & $\mathrm{~N}$ \\
\hline $8 \mathrm{H}-1$ & $145.0-147.0$ & 62.65 & MAG & $30 \mathrm{~A}$ & 356.9 & -66.8 & 3.1266 & $\mathrm{~N}$ \\
\hline $8 \mathrm{H}-2$ & $42.0-44.0$ & 63.12 & MAG & $00 \mathrm{~A}$ & 86.2 & -78.8 & 36.4000 & $\mathrm{~N}$ \\
\hline $8 \mathrm{H}-2$ & $42.0-44.0$ & 63.12 & MAG & $15 \mathrm{~A}$ & 34.2 & -79.9 & 16.2534 & $\mathrm{~N}$ \\
\hline $8 \mathrm{H}-2$ & $42.0-44.0$ & 63.12 & MAG & $22 \mathrm{~A}$ & 16.5 & -77.1 & 14.0460 & $\mathrm{~N}$ \\
\hline $8 \mathrm{H}-2$ & $42.0-44.0$ & 63.12 & MAG & $30 \mathrm{~A}$ & 19.7 & -77.0 & 9.4859 & $\mathrm{~N}$ \\
\hline $8 \mathrm{H}-2$ & $93.0-95.0$ & 63.63 & MAG & $00 \mathrm{~A}$ & 87.8 & -70.9 & 14.1000 & $\mathrm{~N}$ \\
\hline $8 \mathrm{H}-2$ & $93.0-95.0$ & 63.63 & MAG & $30 \mathrm{~A}$ & 16.3 & -76.7 & 1.7787 & $\mathrm{~N}$ \\
\hline $8 \mathrm{H}-2$ & $136.0-138.0$ & 64.06 & MAG & $00 \mathrm{~A}$ & 261.7 & +79.4 & 16.0000 & $\mathbf{R}$ \\
\hline $8 \mathrm{H}-2$ & $136.0-138.0$ & 64.06 & MAG & $15 \mathrm{~A}$ & 183.6 & +71.6 & 12.0487 & $\mathbf{R}$ \\
\hline $8 \mathrm{H}-2$ & $136.0-138.0$ & 64.06 & MAG & $22 \mathrm{~A}$ & 183.3 & +76.1 & 9.4065 & $\mathbf{R}$ \\
\hline $8 \mathrm{H}-2$ & $136.0-138.0$ & 64.06 & MAG & $30 \mathrm{~A}$ & 180.2 & +72.3 & 6.4756 & $\mathbf{R}$ \\
\hline $8 \mathrm{H}-3$ & $29.0-31.0$ & 64.49 & MAG & $00 \mathrm{~A}$ & 255.8 & +84.4 & 3.6600 & $\mathbf{R}$ \\
\hline $8 \mathrm{H}-3$ & $29.0-31.0$ & 64.49 & MAG & $15 \mathrm{~A}$ & 274.6 & +70.1 & 3.6396 & $\mathbf{R}$ \\
\hline $8 \mathrm{H}-3$ & $29.0-31.0$ & 64.49 & MAG & $22 \mathrm{~A}$ & 271.2 & +67.8 & 2.7565 & $\mathbf{R}$ \\
\hline $8 \mathrm{H}-3$ & $29.0-31.0$ & 64.49 & MAG & $30 \mathrm{~A}$ & 246.5 & +72.9 & 1.9186 & $\mathbf{R}$ \\
\hline $8 \mathrm{H}-3$ & $77.0-79.0$ & 64.97 & MAG & $00 \mathrm{~A}$ & 85.1 & -74.6 & 20.2000 & $\mathrm{~N}$ \\
\hline $8 \mathrm{H}-3$ & $77.0-79.0$ & 64.97 & MAG & $15 \mathrm{~A}$ & 359.8 & -1.6 & 908.2716 & $\mathrm{~N}$ \\
\hline $8 \mathrm{H}-3$ & $77.0-79.0$ & 64.97 & MAG & $22 \mathrm{~A}$ & 14.3 & -73.6 & 7.0726 & $\mathrm{~N}$ \\
\hline $8 \mathrm{H}-3$ & $77.0-79.0$ & 64.97 & MAG & $30 \mathrm{~A}$ & 37.3 & -72.5 & 4.5725 & $\mathrm{~N}$ \\
\hline $8 \mathrm{H}-3$ & $134.0-136.0$ & 65.54 & MAG & $00 \mathrm{~A}$ & 89.5 & -58.6 & 6.4500 & A \\
\hline $8 \mathrm{H}-3$ & $134.0-136.0$ & 65.54 & MAG & $15 \mathrm{~A}$ & 359.8 & -1.1 & 101.2426 & A \\
\hline $8 \mathrm{H}-3$ & $134.0-136.0$ & 65.54 & MAG & $22 \mathrm{~A}$ & 332.9 & +38.0 & 0.5769 & A \\
\hline $8 \mathrm{H}-3$ & $134.0-136.0$ & 65.54 & MAG & $30 \mathrm{~A}$ & 329.5 & +0.2 & 0.2383 & A \\
\hline $8 \mathrm{H}-4$ & $36.0-38.0$ & 66.06 & MAG & $00 \mathrm{~A}$ & 266.5 & +24.7 & 5.5700 & $\mathbf{R}$ \\
\hline $8 \mathrm{H}-4$ & $36.0-38.0$ & 66.06 & MAG & $22 \mathrm{~A}$ & 220.7 & +71.7 & 7.3247 & $\mathbf{R}$ \\
\hline $8 \mathrm{H}-4$ & $36.0-38.0$ & 66.06 & MAG & $30 \mathrm{~A}$ & 206.8 & +71.6 & 4.9433 & $\mathbf{R}$ \\
\hline $8 \mathrm{H}-4$ & $78.0-80.0$ & 66.48 & MAG & $00 \mathrm{~A}$ & 267.1 & +35.1 & 6.3500 & $\mathbf{R}$ \\
\hline $8 \mathrm{H}-4$ & $78.0-80.0$ & 66.48 & MAG & $15 \mathrm{~A}$ & 228.9 & +71.2 & 9.5413 & $\mathbf{R}$ \\
\hline $8 \mathrm{H}-4$ & $78.0-80.0$ & 66.48 & MAG & $22 \mathrm{~A}$ & 228.2 & +69.6 & 7.2902 & $\mathbf{R}$ \\
\hline $8 \mathrm{H}-4$ & $78.0-80.0$ & 66.48 & MAG & $30 \mathrm{~A}$ & 203.7 & +69.9 & 4.9918 & R \\
\hline $8 \mathrm{H}-4$ & $121.0-123.0$ & 66.91 & MAG & $00 \mathrm{~A}$ & 90.1 & -4.4 & 4.8600 & A \\
\hline $8 \mathrm{H}-4$ & $121.0-123.0$ & 66.91 & MAG & $22 \mathrm{~A}$ & 194.2 & +68.1 & 3.7865 & A \\
\hline $8 \mathrm{H}-4$ & $121.0-123.0$ & 66.91 & MAG & $30 \mathrm{~A}$ & 156.7 & +70.3 & 2.6281 & A \\
\hline $8 \mathrm{H}-7$ & $7.0-9.0$ & 67.02 & MAG & $00 \mathrm{~A}$ & 269.8 & -35.2 & 7.4000 & $\mathrm{~N}$ \\
\hline $8 \mathrm{H}-7$ & $7.0-9.0$ & 67.02 & MAG & $22 \mathrm{~A}$ & 309.6 & -17.5 & 2.4442 & $\mathrm{~N}$ \\
\hline
\end{tabular}


Appendix (continued).

\begin{tabular}{|c|c|c|c|c|c|c|c|c|}
\hline Core, section & $\begin{array}{c}\text { Interval } \\
\text { (cm) }\end{array}$ & $\begin{array}{l}\text { Depth } \\
\text { (mbsf) }\end{array}$ & S.T. & Demag level & Decl. & Incl. & Intensity & Polarity \\
\hline \multicolumn{9}{|l|}{ 119-744A-(Cont.) } \\
\hline $8 \mathrm{H}-7$ & $7.0-9.0$ & 67.02 & MAG & $30 \mathrm{~A}$ & 316.6 & -1.0 & 0.9953 & $\mathrm{~N}$ \\
\hline $9 \mathrm{H}-1$ & $19.0-21.0$ & 70.89 & MAG & $00 \mathrm{~A}$ & 94.8 & -78.0 & 4.4500 & $\mathrm{~N}$ \\
\hline $9 \mathrm{H}-1$ & $19.0-21.0$ & 70.89 & MAG & $15 \mathrm{~A}$ & 294.9 & -83.8 & 2.4337 & $\mathrm{~N}$ \\
\hline $9 \mathrm{H}-\mathrm{I}$ & $19.0-21.0$ & 70.89 & MAG & $22 \mathrm{~A}$ & 316.0 & -82.4 & 1.8755 & $N$ \\
\hline $9 \mathrm{H}-1$ & $19.0-21.0$ & 70.89 & MAG & $30 \mathrm{~A}$ & 311.6 & -86.7 & 1.8075 & $\mathrm{~N}$ \\
\hline $9 \mathrm{H}-1$ & $53.0-55.0$ & 71.23 & MAG & $00 \mathrm{~A}$ & 101.0 & -79.3 & 7.0800 & $\mathrm{~N}$ \\
\hline $9 \mathrm{H}-1$ & $53.0-55.0$ & 71.23 & MAG & $15 \mathrm{~A}$ & 214.1 & -81.0 & 3.8329 & $\mathrm{~N}$ \\
\hline $9 \mathrm{H}-1$ & $53.0-55.0$ & 71.23 & MAG & $22 \mathrm{~A}$ & 205.1 & -82.2 & 2.7218 & $\mathrm{~N}$ \\
\hline $9 \mathrm{H}-1$ & $53.0-55.0$ & 71.23 & MAG & $30 \mathrm{~A}$ & 134.3 & -85.4 & 1.7955 & $\mathrm{~N}$ \\
\hline $9 \mathrm{H}-1$ & $82.0-84.0$ & 71.52 & MAG & $00 \mathrm{~A}$ & 124.2 & -87.0 & 8.6200 & $\mathrm{~N}$ \\
\hline $9 \mathrm{H}-1$ & $82.0-84.0$ & 71.52 & MAG & $15 \mathrm{~A}$ & 251.4 & -74.8 & 5.1428 & $\mathrm{~N}$ \\
\hline $9 \mathrm{H}-1$ & $82.0-84.0$ & 71.52 & MAG & $22 \mathrm{~A}$ & 244.7 & -77.5 & 3.7780 & $\mathrm{~N}$ \\
\hline $9 \mathrm{H}-1$ & $82.0-84.0$ & 71.52 & MAG & $30 \mathrm{~A}$ & 237.6 & -77.7 & 2.3223 & $\mathbf{N}$ \\
\hline $9 \mathrm{H}-7$ & $10.0-12.0$ & 71.75 & MAG & $00 \mathrm{~A}$ & 270.0 & -5.4 & 3.0900 & A \\
\hline $9 \mathrm{H}-7$ & $10.0-12.0$ & 71.75 & MAG & $15 \mathrm{~A}$ & 342.9 & -54.8 & 0.5284 & A \\
\hline $9 \mathrm{H}-7$ & $10.0-12.0$ & 71.75 & MAG & $30 \mathrm{~A}$ & 67.9 & -51.3 & 0.2728 & A \\
\hline $10 \mathrm{H}-1$ & $36.0-88.0$ & 80.56 & MAG & $00 \mathrm{~A}$ & 89.4 & -69.0 & 27.7000 & $\mathrm{~N}$ \\
\hline $10 \mathrm{H}-1$ & $36.0-88.0$ & 80.56 & MAG & $15 \mathrm{~A}$ & 98.0 & -77.0 & 12.0573 & $\mathrm{~N}$ \\
\hline $10 \mathrm{H}-1$ & $36.0-88.0$ & 80.56 & MAG & $30 \mathrm{~A}$ & 92.9 & -75.5 & 6.1832 & $\mathbf{N}$ \\
\hline $10 \mathrm{H}-2$ & $18.0-20.0$ & 81.88 & MAG & $00 \mathrm{~A}$ & 88.8 & -48.2 & 4.8000 & A \\
\hline $10 \mathrm{H}-2$ & $18.0-20.0$ & 81.88 & MAG & $15 \mathrm{~A}$ & 302.0 & +58.3 & 3.2306 & A \\
\hline $10 \mathrm{H}-2$ & $18.0-20.0$ & 81.88 & MAG & $30 \mathrm{~A}$ & 316.3 & +73.9 & 1.8232 & A \\
\hline $10 \mathrm{H}-2$ & $71.0-73.0$ & 82.41 & MAG & $00 \mathrm{~A}$ & 88.6 & +50.5 & 4.4500 & D \\
\hline $10 \mathrm{H}-2$ & $71.0-73.0$ & 82.41 & MAG & $15 \mathrm{~A}$ & 281.1 & +74.4 & 6.3394 & $\mathbf{R}$ \\
\hline $10 \mathrm{H}-2$ & $71.0-73.0$ & 82.41 & MAG & $22 \mathrm{~A}$ & 285.9 & +75.5 & 5.0927 & $\mathbf{R}$ \\
\hline $10 \mathrm{H}-2$ & $71.0-73.0$ & 82.41 & MAG & $30 \mathrm{~A}$ & 286.9 & +80.0 & 3.7528 & $\mathbf{R}$ \\
\hline $10 \mathrm{H}-2$ & $141.0-143.0$ & 83.11 & MAG & $00 \mathrm{~A}$ & 88.4 & +59.1 & 4.0200 & $\mathbf{R}$ \\
\hline $10 \mathrm{H}-2$ & $141.0-143.0$ & 83.11 & MAG & $15 \mathrm{~A}$ & 281.4 & +76.1 & 6.1966 & $\mathbf{R}$ \\
\hline $10 \mathrm{H}-2$ & $141.0-143.0$ & 83.11 & MAG & $22 \mathrm{~A}$ & 290.8 & +74.5 & 4.7042 & $\mathbf{R}$ \\
\hline $10 \mathrm{H}-2$ & $141.0-143.0$ & 83.11 & MAG & $30 \mathrm{~A}$ & 306.8 & +79.1 & 3.6072 & $\mathbf{R}$ \\
\hline $10 \mathrm{H}-3$ & $6.0-8.0$ & 83.26 & MAG & $00 \mathrm{~A}$ & 92.8 & -65.5 & 8.2200 & $N$ \\
\hline $10 \mathrm{H}-3$ & $6.0-8.0$ & 83.26 & MAG & $15 \mathrm{~A}$ & 27.8 & -80.1 & 2.9647 & $\mathbf{N}$ \\
\hline $10 \mathrm{H}-3$ & $6.0-8.0$ & 83.26 & MAG & $22 \mathrm{~A}$ & 58.8 & -71.3 & 2.3054 & $\mathrm{~N}$ \\
\hline $10 \mathrm{H}-3$ & $6.0-8.0$ & 83.26 & MAG & $30 \mathrm{~A}$ & 76.4 & -76.9 & 1.6738 & $\mathbf{N}$ \\
\hline $10 \mathrm{H}-3$ & $53.0-55.0$ & 83.73 & MAG & $00 \mathrm{~A}$ & 110.1 & -58.8 & 6.6624 & $N$ \\
\hline $10 \mathrm{H}-3$ & $53.0-55.0$ & 83.73 & MAG & $05 \mathrm{~A}$ & 97.0 & -70.5 & 4.8331 & $\mathrm{~N}$ \\
\hline $10 \mathrm{H}-3$ & $53.0-55.0$ & 83.73 & MAG & $10 \mathrm{~A}$ & 102.8 & -74.7 & 4.0647 & $\mathbf{N}$ \\
\hline $10 \mathrm{H}-3$ & $53.0-55.0$ & 83.73 & MAG & $30 \mathrm{~A}$ & 84.1 & -74.3 & 1.6487 & $\mathrm{~N}$ \\
\hline $10 \mathrm{H}-3$ & $53.0-55.0$ & 83.73 & MAG & $50 \mathrm{~A}$ & 50.8 & -83.8 & 0.5072 & $\mathbf{N}$ \\
\hline $10 \mathrm{H}-3$ & $115.0-117.0$ & 84.35 & MAG & $00 \mathrm{~A}$ & 90.6 & -64.8 & 7.8300 & N \\
\hline $10 \mathrm{H}-3$ & $115.0-117.0$ & 84.35 & MAG & $15 \mathrm{~A}$ & 70.7 & -73.9 & 3.8529 & $\mathrm{~N}$ \\
\hline $10 \mathrm{H}-3$ & $115.0-117.0$ & 84.35 & MAG & $22 \mathrm{~A}$ & 60.4 & -70.6 & 2.9102 & $\mathrm{~N}$ \\
\hline $10 \mathrm{H}-3$ & $115.0-117.0$ & 84.35 & MAG & $30 \mathrm{~A}$ & 68.0 & -68.1 & 2.1102 & N \\
\hline $10 \mathrm{H}-4$ & $12.0-14.0$ & 84.82 & MAG & $00 \mathrm{~A}$ & 91.4 & -65.8 & 17.7000 & $\mathrm{~N}$ \\
\hline $10 \mathrm{H}-4$ & $12.0-14.0$ & 84.82 & MAG & $15 \mathrm{~A}$ & 99.0 & -75.8 & 8.6757 & $\mathrm{~N}$ \\
\hline $10 \mathrm{H}-4$ & $12.0-14.0$ & 84.82 & MAG & $22 \mathrm{~A}$ & 104.6 & -78.4 & 6.1523 & $\mathbf{N}$ \\
\hline $10 \mathrm{H}-4$ & $12.0-14.0$ & 84.82 & MAG & $30 \mathrm{~A}$ & 100.5 & -70.6 & 4.3245 & $\mathrm{~N}$ \\
\hline $10 \mathrm{H}-4$ & $65.0-67.0$ & 85.35 & MAG & $00 \mathrm{~A}$ & 92.3 & -67.7 & 19.8540 & $N$ \\
\hline $10 \mathrm{H}-4$ & $65.0-67.0$ & 85.35 & MAG & $10 \mathrm{~A}$ & 102.4 & -74.1 & 4.0121 & $\mathbf{N}$ \\
\hline $10 \mathrm{H}-4$ & $65.0-67.0$ & 85.35 & MAG & $20 \mathrm{~A}$ & 99.1 & -75.7 & 8.1052 & $\mathrm{~N}$ \\
\hline $10 \mathrm{H}-4$ & $65.0-67.0$ & 85.35 & MAG & $30 \mathrm{~A}$ & 83.9 & -73.1 & 4.8392 & $\mathbf{N}$ \\
\hline $10 \mathrm{H}-4$ & $65.0-67.0$ & 85.35 & MAG & $40 \mathrm{~A}$ & 83.4 & -72.2 & 2.6583 & $\mathbf{N}$ \\
\hline $10 \mathrm{H}-4$ & $65.0-67.0$ & 85.35 & MAG & $50 \mathrm{~A}$ & 115.8 & -68.4 & 1.7885 & $\mathbf{N}$ \\
\hline $10 \mathrm{H}-4$ & $104.0-106.0$ & 85.74 & MAG & $00 \mathrm{~A}$ & 91.5 & -65.1 & 17.0000 & $N$ \\
\hline $10 \mathrm{H}-4$ & $104.0-106.0$ & 85.74 & MAG & $15 \mathrm{~A}$ & 126.2 & -75.0 & 8.2765 & $\mathbf{N}$ \\
\hline $10 \mathrm{H}-4$ & $104.0-106.0$ & 85.74 & MAG & $22 \mathrm{~A}$ & 131.0 & -76.9 & 5.8060 & $\mathrm{~N}$ \\
\hline $10 \mathrm{H}-4$ & $104.0-106.0$ & 85.74 & MAG & $30 \mathrm{~A}$ & 121.2 & -71.4 & 3.9856 & $\mathrm{~N}$ \\
\hline $10 \mathrm{H}-5$ & $10.0-12.0$ & 86.30 & MAG & $00 \mathrm{~A}$ & 90.6 & -62.5 & 14.6000 & $\mathbf{N}$ \\
\hline $10 \mathrm{H}-5$ & $10.0-12.0$ & 86.30 & MAG & $15 \mathrm{~A}$ & 104.5 & -75.2 & 5.8714 & $\mathrm{~N}$ \\
\hline $10 \mathrm{H}-5$ & $10.0-12.0$ & 86.30 & MAG & $22 \mathrm{~A}$ & 114.2 & -79.0 & 4.4276 & $\mathrm{~N}$ \\
\hline $10 \mathrm{H}-5$ & $10.0-12.0$ & 86.30 & MAG & $30 \mathrm{~A}$ & 107.2 & -73.6 & 3.1125 & $\mathrm{~N}$ \\
\hline $10 \mathrm{H}-5$ & $62.0-64.0$ & 86.82 & MAG & $00 \mathrm{~A}$ & 91.5 & -66.7 & 13.0000 & $N$ \\
\hline $10 \mathrm{H}-5$ & $62.0-64.0$ & 86.82 & MAG & $15 \mathrm{~A}$ & 120.4 & -78.6 & 6.8099 & $\mathrm{~N}$ \\
\hline $10 \mathrm{H}-5$ & $62.0-64.0$ & 86.82 & MAG & $22 \mathrm{~A}$ & 114.4 & -80.1 & 4.9504 & $\mathrm{~N}$ \\
\hline $10 \mathrm{H}-5$ & $62.0-64.0$ & 86.82 & MAG & $30 \mathrm{~A}$ & 118.3 & -79.3 & 3.5532 & $\mathrm{~N}$ \\
\hline $10 \mathrm{H}-5$ & $128.0-130.0$ & 87.48 & MAG & $00 \mathrm{~A}$ & 90.7 & -67.5 & 14.4000 & $\mathrm{~N}$ \\
\hline $10 \mathrm{H}-5$ & $128.0-130.0$ & 87.48 & MAG & $15 \mathrm{~A}$ & 86.5 & -80.1 & 6.5844 & N \\
\hline $10 \mathrm{H}-5$ & $128.0-130.0$ & 87.48 & MAG & $22 \mathrm{~A}$ & 73.9 & -79.5 & 4.9805 & $\mathrm{~N}$ \\
\hline $10 \mathrm{H}-6$ & $14.0-16.0$ & 87.84 & MAG & $00 \mathrm{~A}$ & 270.7 & -53.1 & 3.2900 & $\mathbf{N}$ \\
\hline $10 \mathrm{H}-6$ & $14.0-16.0$ & 87.84 & MAG & $15 \mathrm{~A}$ & 41.9 & -63.1 & 2.0833 & $N$ \\
\hline $10 \mathrm{H}-6$ & $14.0-16.0$ & 87.84 & MAG & $22 \mathrm{~A}$ & 38.2 & -59.6 & 1.9867 & $\mathrm{~N}$ \\
\hline $10 \mathrm{H}-6$ & $14.0-16.0$ & 87.84 & MAG & $30 \mathrm{~A}$ & 57.2 & -60.1 & 1.2907 & $\mathbf{N}$ \\
\hline $10 \mathrm{H}-6$ & $110.0-112.0$ & 88.80 & MAG & $00 \mathrm{~A}$ & 90.9 & -68.0 & 21.3000 & $\mathbf{N}$ \\
\hline $10 \mathrm{H}-6$ & $110.0-112.0$ & 88.80 & MAG & $15 \mathrm{~A}$ & 83.4 & -78.7 & 9.3661 & $N$ \\
\hline $10 \mathrm{H}-6$ & $110.0-112.0$ & 88.80 & MAG & $22 \mathrm{~A}$ & 88.1 & -81.3 & 6.5892 & $\mathrm{~N}$ \\
\hline $10 \mathrm{H}-6$ & $110.0-112.0$ & 88.80 & MAG & $30 \mathrm{~A}$ & 71.5 & -76.5 & 4.7968 & $\mathrm{~N}$ \\
\hline
\end{tabular}


Appendix (continued).

\begin{tabular}{|c|c|c|c|c|c|c|c|c|}
\hline Core, section & $\begin{array}{l}\text { Interval } \\
(\mathrm{cm})\end{array}$ & $\begin{array}{l}\text { Depth } \\
\text { (mbsf) }\end{array}$ & S.T. & Demag level & Decl. & Incl. & Intensity & Polarity \\
\hline \multicolumn{9}{|l|}{ 119-744A-(Cont.) } \\
\hline $10 \mathrm{H}-6$ & $143.0-145.0$ & 89.13 & MAG & $00 \mathrm{~A}$ & 92.3 & -65.5 & 28.3000 & $\mathrm{~N}$ \\
\hline $10 \mathrm{H}-6$ & $143.0-145.0$ & 89.13 & MAG & $15 \mathrm{~A}$ & 109.1 & -73.5 & 13.6093 & $\mathrm{~N}$ \\
\hline $10 \mathrm{H}-6$ & $143.0-145.0$ & 89.13 & MAG & $22 \mathrm{~A}$ & 107.9 & -77.5 & 9.7409 & $\mathrm{~N}$ \\
\hline $10 \mathrm{H}-6$ & $143.0-145.0$ & 89.13 & MAG & $30 \mathrm{~A}$ & 101.0 & -73.3 & 6.7756 & $\mathrm{~N}$ \\
\hline $10 \mathrm{H}-7$ & $11.0-13.0$ & 89.31 & MAG & $00 \mathrm{~A}$ & 90.2 & -64.3 & 24.5000 & $\mathbf{N}$ \\
\hline $10 \mathrm{H}-7$ & $11.0-13.0$ & 89.31 & MAG & $15 \mathrm{~A}$ & 89.7 & -73.7 & 12.5207 & $\mathbf{N}$ \\
\hline $10 \mathrm{H}-7$ & $11.0-13.0$ & 89.31 & MAG & $30 \mathrm{~A}$ & 93.4 & -73.4 & 6.6513 & $\mathbf{N}$ \\
\hline $10 \mathrm{H}-7$ & $12.0-14.0$ & 89.32 & MAG & $00 \mathrm{~A}$ & 261.0 & +6.0 & 5.8600 & A \\
\hline $10 \mathrm{H}-7$ & $12.0-14.0$ & 89.32 & MAG & $22 \mathrm{~A}$ & 207.0 & -38.1 & 1.8913 & A \\
\hline $10 \mathrm{H}-7$ & $12.0-14.0$ & 89.32 & MAG & $30 \mathrm{~A}$ & 198.2 & -43.9 & 1.2538 & A \\
\hline $10 \mathrm{H}-7$ & $33.0-35.0$ & 89.53 & MAG & $00 \mathrm{~A}$ & 92.3 & -63.2 & 22.1000 & $\mathrm{~N}$ \\
\hline $10 \mathrm{H}-7$ & $33.0-35.0$ & 89.53 & MAG & $15 \mathrm{~A}$ & 104.8 & -75.8 & 12.7254 & $\mathrm{~N}$ \\
\hline $10 \mathrm{H}-7$ & $33.0-35.0$ & 89.53 & MAG & $22 \mathrm{~A}$ & 98.7 & -77.7 & 8.9350 & $\mathrm{~N}$ \\
\hline $10 \mathrm{H}-7$ & $33.0-35.0$ & 89.53 & MAG & $30 \mathrm{~A}$ & 107.4 & -74.2 & 6.4068 & $\mathrm{~N}$ \\
\hline $10 \mathrm{H}-7$ & $58.0-60.0$ & 89.78 & MAG & $00 \mathrm{~A}$ & 90.6 & -62.8 & 25.8000 & $\mathbf{N}$ \\
\hline $10 \mathrm{H}-7$ & $58.0-60.0$ & 89.78 & MAG & $15 \mathrm{~A}$ & 91.8 & -76.2 & 13.8315 & $\mathrm{~N}$ \\
\hline $10 \mathrm{H}-7$ & $58.0-60.0$ & 89.78 & MAG & $22 \mathrm{~A}$ & 95.7 & -78.5 & 9.7904 & $\mathrm{~N}$ \\
\hline $10 \mathrm{H}-7$ & $58.0-60.0$ & 89.78 & MAG & $30 \mathrm{~A}$ & 90.1 & -76.0 & 6.9428 & $\mathbf{N}$ \\
\hline $11 \mathrm{H}-1$ & $51.0-53.0$ & 90.21 & MAG & $00 \mathrm{~A}$ & 77.4 & -86.0 & 15.0000 & $\mathbf{N}$ \\
\hline $11 \mathrm{H}-1$ & $51.0-53.0$ & 90.21 & MAG & $15 \mathrm{~A}$ & 328.2 & -72.5 & 7.9356 & $\mathbf{N}$ \\
\hline $11 \mathrm{H}-1$ & $51.0-53.0$ & 90.21 & MAG & $22 \mathrm{~A}$ & 334.8 & -72.7 & 5.7155 & $\mathrm{~N}$ \\
\hline $11 \mathrm{H}-1$ & $51.0-53.0$ & 90.21 & MAG & $30 \mathrm{~A}$ & 357.3 & -74.3 & 4.1816 & $\mathrm{~N}$ \\
\hline $11 \mathrm{H}-1$ & $98.0-100.0$ & 90.68 & MAG & $00 \mathrm{~A}$ & 83.9 & -82.7 & 19.4000 & $\mathbf{N}$ \\
\hline $11 \mathrm{H}-1$ & $98.0-100.0$ & 90.68 & MAG & $15 \mathrm{~A}$ & 333.7 & -75.2 & 9.9353 & $\mathrm{~N}$ \\
\hline $11 \mathrm{H}-1$ & $98.0-100.0$ & 90.68 & MAG & $22 \mathrm{~A}$ & 342.2 & -74.0 & 7.1466 & $\mathrm{~N}$ \\
\hline $11 \mathrm{H}-1$ & $98.0-100.0$ & 90.68 & MAG & $30 \mathrm{~A}$ & 352.9 & -74.1 & 4.7635 & $\mathbf{N}$ \\
\hline $11 \mathrm{H}-1$ & $144.0-146.0$ & 91.14 & MAG & $00 \mathrm{~A}$ & 50.3 & -87.5 & 15.0000 & $\mathrm{~N}$ \\
\hline $11 \mathrm{H}-1$ & $144.0-146.0$ & 91.14 & MAG & $15 \mathrm{~A}$ & 331.4 & -65.7 & 8.0239 & $\mathbf{N}$ \\
\hline $11 \mathrm{H}-1$ & $144.0-146.0$ & 91.14 & MAG & $22 \mathrm{~A}$ & 334.4 & -66.0 & 5.8398 & $\mathrm{~N}$ \\
\hline $11 \mathrm{H}-1$ & $144.0-146.0$ & 91.14 & MAG & $30 \mathrm{~A}$ & 339.6 & -66.2 & 3.8940 & $\mathrm{~N}$ \\
\hline $11 \mathrm{H}-2$ & $20.0-22.0$ & 91.40 & MAG & $00 \mathrm{~A}$ & 90.4 & -80.9 & 10.6000 & $\mathbf{N}$ \\
\hline $11 \mathrm{H}-2$ & $20.0-22.0$ & 91.40 & MAG & $22 \mathrm{~A}$ & 315.2 & -68.6 & 4.0782 & $\mathrm{~N}$ \\
\hline $11 \mathrm{H}-2$ & $20.0-22.0$ & 91.40 & MAG & $30 \mathrm{~A}$ & 348.3 & -78.3 & 2.5301 & $\mathbf{N}$ \\
\hline $11 \mathrm{H}-2$ & $74.0-76.0$ & 91.94 & MAG & $00 \mathrm{~A}$ & 89.0 & -74.9 & 12.0000 & $\mathrm{~N}$ \\
\hline $11 \mathrm{H}-2$ & $74.0-76.0$ & 91.94 & MAG & $15 \mathrm{~A}$ & 304.8 & -70.5 & 5.6584 & $\mathbf{N}$ \\
\hline $11 \mathrm{H}-2$ & $74.0-76.0$ & 91.94 & MAG & $22 \mathrm{~A}$ & 335.0 & -74.3 & 3.1353 & $\mathrm{~N}$ \\
\hline $11 \mathrm{H}-2$ & $74.0-76.0$ & 91.94 & MAG & $30 \mathrm{~A}$ & 10.8 & -78.2 & 2.0711 & $\mathbf{N}$ \\
\hline $11 \mathrm{H}-2$ & $128.0-130.0$ & 92.48 & MAG & $00 \mathrm{~A}$ & 81.8 & -77.1 & 8.5200 & $\mathrm{~N}$ \\
\hline $11 \mathrm{H}-2$ & $128.0-130.0$ & 92.48 & MAG & $15 \mathrm{~A}$ & 336.4 & -70.4 & 2.8536 & $\mathbf{N}$ \\
\hline $11 \mathrm{H}-2$ & $128.0-130.0$ & 92.48 & MAG & $22 \mathrm{~A}$ & 334.4 & -65.9 & 2.1180 & $\mathrm{~N}$ \\
\hline $11 \mathrm{H}-2$ & $128.0-130.0$ & 92.48 & MAG & $30 \mathrm{~A}$ & 2.8 & -70.1 & 1.4180 & $\mathbf{N}$ \\
\hline $11 \mathrm{H}-3$ & $41.0-43.0$ & 93.11 & MAG & $00 \mathrm{~A}$ & 90.2 & -45.8 & 4.7100 & A \\
\hline $11 \mathrm{H}-3$ & $41.0-43.0$ & 93.11 & MAG & $15 \mathrm{~A}$ & 210.5 & +74.5 & 2.1311 & A \\
\hline $11 \mathrm{H}-3$ & $79.0-81.0$ & 93.49 & MAG & $00 \mathrm{~A}$ & 88.2 & -44.2 & 4.6500 & A \\
\hline IIH-3 & $79.0-81.0$ & 93.49 & MAG & $15 \mathrm{~A}$ & 169.7 & +85.7 & 2.2851 & A \\
\hline $11 \mathrm{H}-3$ & $79.0-81.0$ & 93.49 & MAG & $30 \mathrm{~A}$ & 140.6 & +82.0 & 1.2572 & A \\
\hline $11 \mathrm{H}-3$ & $125.0-127.0$ & 93.95 & MAG & $00 \mathrm{~A}$ & 90.1 & -31.3 & 6.4500 & $\mathrm{~N}$ \\
\hline $11 \mathrm{H}-3$ & $125.0-127.0$ & 93.95 & MAG & $15 \mathrm{~A}$ & 131.9 & -69.3 & 0.4636 & $\mathrm{~N}$ \\
\hline $11 \mathrm{H}-4$ & $20.0-22.0$ & 94.40 & MAG & $00 \mathrm{~A}$ & 90.4 & -58.8 & 8.4600 & A \\
\hline $11 \mathrm{H}-4$ & $20.0-22.0$ & 94.40 & MAG & $15 \mathrm{~A}$ & 194.9 & +54.9 & 1.6684 & A \\
\hline $11 \mathrm{H}-4$ & $66.0-68.0$ & 94.86 & MAG & $00 \mathrm{~A}$ & 90.3 & -60.6 & 10.9000 & $\mathrm{~N}$ \\
\hline $11 \mathrm{H}-4$ & $66.0-68.0$ & 94.86 & MAG & $22 \mathrm{~A}$ & 15.0 & -68.2 & 2.3357 & $\mathrm{~N}$ \\
\hline $11 \mathrm{H}-4$ & $66.0-68.0$ & 94.86 & MAG & $15 \mathrm{~A}$ & 350.8 & -70.4 & 3.4692 & $\mathrm{~N}$ \\
\hline $11 \mathrm{H}-4$ & $123.0-125.0$ & 95.43 & MAG & $00 \mathrm{~A}$ & 91.3 & -75.4 & 7.1800 & $\mathrm{~N}$ \\
\hline $11 \mathrm{H}-4$ & $123.0-125.0$ & 95.43 & MAG & $22 \mathrm{~A}$ & 325.2 & -61.4 & 0.8430 & $\mathrm{~N}$ \\
\hline $11 \mathrm{H}-4$ & $123.0-125.0$ & 95.43 & MAG & $15 \mathrm{~A}$ & 326.1 & -56.0 & 1.1617 & $\mathbf{N}$ \\
\hline $11 \mathrm{H}-5$ & $40.0-42.0$ & 96.10 & MAG & $00 \mathrm{~A}$ & 91.9 & -75.9 & 17.7000 & $\mathrm{~N}$ \\
\hline $11 \mathrm{H}-5$ & $40.0-42.0$ & 96.10 & MAG & $22 \mathrm{~A}$ & 16.1 & -87.9 & 4.1248 & $\mathrm{~N}$ \\
\hline $11 \mathrm{H}-5$ & $40.0-42.0$ & 96.10 & MAG & $15 \mathrm{~A}$ & 270.2 & -89.2 & 5.9704 & $\mathrm{~N}$ \\
\hline $11 \mathrm{H}-5$ & $76.0-78.0$ & 96.46 & MAG & $00 \mathrm{~A}$ & 91.4 & -72.2 & 14.6000 & $\mathrm{~N}$ \\
\hline $11 \mathrm{H}-5$ & $76.0-78.0$ & 96.46 & MAG & $22 \mathrm{~A}$ & 23.7 & -69.2 & 2.5126 & $\mathrm{~N}$ \\
\hline $11 \mathrm{H}-5$ & $76.0-78.0$ & 96.46 & MAG & $15 \mathrm{~A}$ & 28.6 & -76.1 & 3.6226 & $\mathrm{~N}$ \\
\hline $11 \mathrm{H}-5$ & $120.0-122.0$ & 96.90 & MAG & $00 \mathrm{~A}$ & 183.4 & -6.6 & 15.7605 & A \\
\hline $11 \mathrm{H}-5$ & $120.0-122.0$ & 96.90 & MAG & $05 \mathrm{~A}$ & 140.1 & +69.5 & 4.0500 & A \\
\hline $11 \mathrm{H}-5$ & $120.0-122.0$ & 96.90 & MAG & $10 \mathrm{~A}$ & 180.2 & +72.2 & 5.8400 & A \\
\hline $11 \mathrm{H}-5$ & $120.0-122.0$ & 96.90 & MAG & $20 \mathrm{~A}$ & 157.1 & +77.8 & 6.0600 & A \\
\hline $11 \mathrm{H}-5$ & $120.0-122.0$ & 96.90 & MAG & $30 \mathrm{~A}$ & 161.1 & +69.7 & 2.9900 & A \\
\hline $11 \mathrm{H}-6$ & $19.0-21.0$ & 97.39 & MAG & $00 \mathrm{~A}$ & 238.5 & -1.7 & 7.8135 & $\mathrm{~N}$ \\
\hline $11 \mathrm{H}-6$ & $19.0-21.0$ & 97.39 & MAG & $05 \mathrm{~A}$ & 308.4 & -28.6 & 2.4107 & $\mathbf{N}$ \\
\hline $11 \mathrm{H}-6$ & $19.0-21.0$ & 97.39 & MAG & $10 \mathrm{~A}$ & 321.9 & -24.7 & 1.2428 & $\mathrm{~N}$ \\
\hline $11 \mathrm{H}-6$ & $19.0-21.0$ & 97.39 & MAG & $20 \mathrm{~A}$ & 319.3 & -44.5 & 0.2642 & $\mathrm{~N}$ \\
\hline $11 \mathrm{H}-6$ & $56.0-58.0$ & 97.76 & MAG & $00 \mathrm{~A}$ & 90.2 & -44.9 & 5.2600 & $\mathrm{~N}$ \\
\hline $11 \mathrm{H}-6$ & $56.0-58.0$ & 97.76 & MAG & $15 \mathrm{~A}$ & 185.9 & -55.0 & 1.3471 & $\mathrm{~N}$ \\
\hline $11 \mathrm{H}-6$ & $56.0-58.0$ & 97.76 & MAG & $22 \mathrm{~A}$ & 125.4 & -72.6 & 0.6121 & $\mathrm{~N}$ \\
\hline $11 \mathrm{H}-6$ & $97.0-99.0$ & 98.17 & MAG & $00 \mathrm{~A}$ & 89.8 & +24.7 & 4.6700 & $\mathbf{R}$ \\
\hline $11 \mathrm{H}-6$ & $97.0-99.0$ & 98.17 & MAG & $22 \mathrm{~A}$ & 212.2 & +78.3 & 6.3008 & $\mathbf{R}$ \\
\hline
\end{tabular}


Appendix (continued).

\begin{tabular}{|c|c|c|c|c|c|c|c|c|}
\hline Core, section & $\begin{array}{c}\text { Interval } \\
(\mathrm{cm})\end{array}$ & $\begin{array}{l}\text { Depth } \\
\text { (mbsf) }\end{array}$ & S.T. & Demag level & Decl. & Incl. & Intensity & Polarity \\
\hline \multicolumn{9}{|l|}{ 119-744A-(Cont.) } \\
\hline $11 \mathrm{H}-6$ & $97.0-99.0$ & 98.17 & MAG & $15 \mathrm{~A}$ & 228.2 & +77.5 & 8.6695 & $\mathbf{R}$ \\
\hline $12 \mathrm{H}-1$ & $78.0-80.0$ & 99.98 & MAG & $00 \mathrm{~A}$ & 90.9 & -63.0 & 13.5000 & $\mathrm{~N}$ \\
\hline $12 \mathrm{H}-1$ & $78.0-80.0$ & 98.98 & MAG & $15 \mathrm{~A}$ & 146.4 & -75.9 & 2.3939 & $\mathrm{~N}$ \\
\hline $12 \mathrm{H}-1$ & $78.0-80.0$ & 99.98 & MAG & $22 \mathrm{~A}$ & 130.4 & -70.9 & 1.5579 & $\mathbf{N}$ \\
\hline $12 \mathrm{H}-1$ & $129.0-131.0$ & 100.49 & MAG & $00 \mathrm{~A}$ & 90.3 & -21.8 & 3.8600 & A \\
\hline $12 \mathrm{H}-1$ & $129.0-131.0$ & 100.49 & MAG & $15 \mathrm{~A}$ & 312.5 & +71.1 & 4.7536 & A \\
\hline $12 \mathrm{H}-2$ & $21.0-23.0$ & 100.91 & MAG & $00 \mathrm{~A}$ & 90.0 & -3.0 & 2.9800 & A \\
\hline $12 \mathrm{H}-2$ & $21.0-23.0$ & 100.91 & MAG & $15 \mathrm{~A}$ & 301.9 & +76.4 & 3.2803 & A \\
\hline $12 \mathrm{H}-2$ & $67.0-69.0$ & 101.37 & MAG & $00 \mathrm{~A}$ & 88.0 & +62.7 & 2.3500 & $\mathbf{R}$ \\
\hline $12 \mathrm{H}-2$ & $67.0-69.0$ & 101.37 & MAG & $15 \mathrm{~A}$ & 331.8 & +78.9 & 4.8202 & $\mathbf{R}$ \\
\hline $12 \mathrm{H}-2$ & $67.0-69.0$ & 101.37 & MAG & $22 \mathrm{~A}$ & 334.0 & +76.2 & 3.5513 & $\mathbf{R}$ \\
\hline $12 \mathrm{H}-2$ & $131.0-133.0$ & 102.01 & MAG & $00 \mathrm{~A}$ & 89.9 & -45.7 & 4.0900 & A \\
\hline $12 \mathrm{H}-2$ & $131.0-133.0$ & 102.01 & MAG & $15 \mathrm{~A}$ & 324.2 & +20.5 & 0.3245 & A \\
\hline $12 \mathrm{H}-3$ & $23.0-25.0$ & 102.43 & MAG & $00 \mathrm{~A}$ & 82.2 & +85.4 & 6.7400 & $\mathbf{R}$ \\
\hline $12 \mathrm{H}-3$ & $23.0-25.0$ & 102.43 & MAG & $15 \mathrm{~A}$ & 321.9 & +73.7 & 8.9141 & $\mathbf{R}$ \\
\hline $12 \mathrm{H}-3$ & $23.0-25.0$ & 102.43 & MAG & $22 \mathrm{~A}$ & 320.9 & +77.0 & 6.0040 & $\mathbf{R}$ \\
\hline $12 \mathrm{H}-3$ & $76.0-78.0$ & 102.96 & MAG & $00 \mathrm{~A}$ & 89.7 & -14.4 & 1.9900 & A \\
\hline $12 \mathrm{H}-3$ & $76.0-78.0$ & 102.96 & MAG & $15 \mathrm{~A}$ & 28.2 & +71.8 & 3.0610 & A \\
\hline $12 \mathrm{H}-3$ & $134.0-136.0$ & 103.54 & MAG & $00 \mathrm{~A}$ & 89.5 & -9.1 & 2.0500 & A \\
\hline $12 \mathrm{H}-3$ & $134.0-136.0$ & 103.54 & MAG & $15 \mathrm{~A}$ & 354.1 & +69.4 & 2.5189 & A \\
\hline $12 \mathrm{H}-4$ & $26.0-28.0$ & 103.96 & MAG & $00 \mathrm{~A}$ & 87.4 & +47.4 & 3.6100 & $\mathbf{R}$ \\
\hline $12 \mathrm{H}-4$ & $26.0-28.0$ & 103.96 & MAG & $15 \mathrm{~A}$ & 24.7 & +66.1 & 6.2196 & $\mathbf{R}$ \\
\hline $12 \mathrm{H}-4$ & $26.0-28.0$ & 103.96 & MAG & $22 \mathrm{~A}$ & 11.6 & +72.2 & 4.3650 & $\mathbf{R}$ \\
\hline $12 \mathrm{H}-4$ & $80.0-82.0$ & 104.50 & MAG & $00 \mathrm{~A}$ & 88.8 & +23.9 & 3.3900 & $\mathbf{R}$ \\
\hline $12 \mathrm{H}-4$ & $80.0-82.0$ & 104.50 & MAG & $15 \mathrm{~A}$ & 66.7 & +44.8 & 6.0240 & $\mathbf{R}$ \\
\hline $12 \mathrm{H}-4$ & $80.0-82.0$ & 104.50 & MAG & $22 \mathrm{~A}$ & 37.0 & +73.1 & 3.8810 & $\mathbf{R}$ \\
\hline $12 \mathrm{H}-4$ & $137.0-139.0$ & 105.07 & MAG & $15 \mathrm{~A}$ & 84.3 & -49.2 & 2.1919 & $\mathbf{N}$ \\
\hline $12 \mathrm{H}-4$ & $137.0-139.0$ & 105.07 & MAG & $00 \mathrm{~A}$ & 90.3 & -44.6 & 4.8700 & $\mathbf{N}$ \\
\hline $12 \mathrm{H}-5$ & $32.0-34.0$ & 105.52 & MAG & $00 \mathrm{~A}$ & 90.8 & -56.4 & 10.0000 & $\mathrm{~N}$ \\
\hline $12 \mathrm{H}-5$ & $32.0-34.0$ & 105.52 & MAG & $15 \mathrm{~A}$ & 116.0 & -71.9 & 4.7476 & $\mathrm{~N}$ \\
\hline $12 \mathrm{H}-5$ & $32.0-34.0$ & 105.52 & MAG & $22 \mathrm{~A}$ & 107.1 & -76.9 & 1.1383 & $\mathrm{~N}$ \\
\hline $12 \mathrm{H}-5$ & $82.0-84.0$ & 106.02 & MAG & $00 \mathrm{~A}$ & 86.9 & +77.8 & 26.2000 & $\mathrm{~N}$ \\
\hline $12 \mathrm{H}-5$ & $82.0-84.0$ & 106.02 & MAG & $15 \mathrm{~A}$ & 178.0 & -77.1 & 5.9391 & $\mathrm{~N}$ \\
\hline $12 \mathrm{H}-5$ & $144.0-146.0$ & 106.64 & MAG & $00 \mathrm{~A}$ & 89.7 & -44.3 & 7.5300 & A \\
\hline $12 \mathrm{H}-5$ & $144.0-146.0$ & 106.64 & MAG & $15 \mathrm{~A}$ & 88.5 & -29.2 & 2.3685 & A \\
\hline $12 \mathrm{H}-5$ & $144.0-146.0$ & 106.64 & MAG & $22 \mathrm{~A}$ & 24.9 & +78.7 & 2.1824 & A \\
\hline $12 \mathrm{H}-6$ & $17.0-19.0$ & 106.87 & MAG & $15 \mathrm{~A}$ & 193.4 & -78.4 & 9.5638 & $\mathrm{~N}$ \\
\hline $12 \mathrm{H}-6$ & $17.0-19.0$ & 106.87 & MAG & $00 \mathrm{~A}$ & 263.5 & -80.9 & 13.6000 & $\mathrm{~N}$ \\
\hline $12 \mathrm{H}-6$ & $17.0-19.0$ & 106.87 & MAG & $22 \mathrm{~A}$ & 177.1 & -74.0 & 2.1130 & $\mathrm{~N}$ \\
\hline $12 \mathrm{H}-6$ & $74.0-76.0$ & 107.44 & MAG & $15 \mathrm{~A}$ & 241.4 & -73.3 & 2.4334 & $\mathrm{~N}$ \\
\hline $12 \mathrm{H}-6$ & $74.0-76.0$ & 107.44 & MAG & $00 \mathrm{~A}$ & 262.3 & -84.0 & 5.8900 & $\mathrm{~N}$ \\
\hline $12 \mathrm{H}-6$ & $74.0-76.0$ & 107.44 & MAG & $22 \mathrm{~A}$ & 307.4 & -71.9 & 0.5000 & $\mathrm{~N}$ \\
\hline $12 \mathrm{H}-6$ & $127.0-129.0$ & 107.97 & MAG & $00 \mathrm{~A}$ & 92.2 & -64.0 & 6.2300 & $\mathrm{~N}$ \\
\hline $12 \mathrm{H}-6$ & $127.0-129.0$ & 107.97 & MAG & $15 \mathrm{~A}$ & 119.6 & -71.1 & 4.3156 & $\mathbf{N}$ \\
\hline $12 \mathrm{H}-6$ & $127.0-129.0$ & 107.97 & MAG & $22 \mathrm{~A}$ & 80.6 & -76.8 & 1.1835 & $\mathrm{~N}$ \\
\hline $12 \mathrm{H}-7$ & $13.0-15.0$ & 108.33 & MAG & $15 \mathrm{~A}$ & 214.3 & -80.8 & 3.7775 & $\mathbf{N}$ \\
\hline $12 \mathrm{H}-7$ & $13.0-15.0$ & 108.33 & MAG & $00 \mathrm{~A}$ & 267.7 & -68.7 & 5.7200 & $\mathrm{~N}$ \\
\hline $12 \mathrm{H}-7$ & $13.0-15.0$ & 108.33 & MAG & $22 \mathrm{~A}$ & 103.5 & -74.2 & 0.9203 & $\mathrm{~N}$ \\
\hline $12 \mathrm{H}-7$ & $58.0-60.0$ & 108.78 & MAG & $00 \mathrm{~A}$ & 267.6 & -73.7 & 9.3200 & $\mathrm{~N}$ \\
\hline $12 \mathrm{H}-7$ & $58.0-60.0$ & 108.78 & MAG & $15 \mathrm{~A}$ & 295.8 & -87.8 & 7.1157 & $\mathrm{~N}$ \\
\hline $12 \mathrm{H}-7$ & $58.0-60.0$ & 108.78 & MAG & $22 \mathrm{~A}$ & 57.6 & -81.2 & 2.4611 & $\mathrm{~N}$ \\
\hline $12 \mathrm{H}-7$ & $14.0-16.0$ & 109.04 & MAG & $00 \mathrm{~A}$ & 269.0 & -49.0 & 7.8900 & $\mathrm{~N}$ \\
\hline $12 \mathrm{H}-7$ & $14.0-16.0$ & 109.04 & MAG & $15 \mathrm{~A}$ & 283.5 & -63.5 & 4.6897 & $\mathbf{N}$ \\
\hline $12 \mathrm{H}-7$ & $14.0-16.0$ & 109.04 & MAG & $22 \mathrm{~A}$ & 280.1 & -46.0 & 1.5910 & $\mathbf{N}$ \\
\hline $13 \mathrm{H}-1$ & $38.0-40.0$ & 109.08 & MAG & $00 \mathrm{~A}$ & 92.2 & -75.2 & 8.4300 & $\mathrm{~N}$ \\
\hline $13 \mathrm{H}-1$ & $38.0-40.0$ & 109.08 & MAG & $15 \mathrm{~A}$ & 184.9 & -77.0 & 7.0902 & $\mathrm{~N}$ \\
\hline $13 \mathrm{H}-1$ & $38.0-40.0$ & 109.08 & MAG & $22 \mathrm{~A}$ & 204.1 & -78.3 & 2.9466 & $\mathrm{~N}$ \\
\hline $13 \mathrm{H}-1$ & $85.0-87.0$ & 109.55 & MAG & $00 \mathrm{~A}$ & 267.9 & -67.2 & 8.4400 & $\mathrm{~N}$ \\
\hline $13 \mathrm{H}-1$ & $85.0-87.0$ & 109.55 & MAG & $15 \mathrm{~A}$ & 243.1 & -73.7 & 8.8361 & $\mathrm{~N}$ \\
\hline $13 \mathrm{H}-1$ & $85.0-87.0$ & 109.55 & MAG & $22 \mathrm{~A}$ & 242.4 & -77.0 & 4.1892 & $\mathrm{~N}$ \\
\hline $13 \mathrm{H}-1$ & $132.0-134.0$ & 110.02 & MAG & $00 \mathrm{~A}$ & 273.5 & +80.7 & 35.0000 & A \\
\hline $13 \mathrm{H}-1$ & $132.0-134.0$ & 110.02 & MAG & $15 \mathrm{~A}$ & 256.6 & -71.4 & 9.9925 & A \\
\hline $13 \mathrm{H}-2$ & $20.0-22.0$ & 110.40 & MAG & $00 \mathrm{~A}$ & 268.3 & -61.6 & 8.2900 & $\mathrm{~N}$ \\
\hline $13 \mathrm{H}-2$ & $20.0-22.0$ & 110.40 & MAG & $15 \mathrm{~A}$ & 276.2 & -65.6 & 6.8343 & $\mathrm{~N}$ \\
\hline $13 \mathrm{H}-2$ & $20.0-22.0$ & 110.40 & MAG & $22 \mathrm{~A}$ & 275.0 & -64.8 & 3.3262 & $\mathrm{~N}$ \\
\hline $13 \mathrm{H}-2$ & $67.0-69.0$ & 110.87 & MAG & $00 \mathrm{~A}$ & 260.3 & -85.6 & 13.8000 & $\mathrm{~N}$ \\
\hline $13 \mathrm{H}-2$ & $67.0-69.0$ & 110.87 & MAG & $15 \mathrm{~A}$ & 249.1 & -85.6 & 13.1261 & $\mathrm{~N}$ \\
\hline $13 \mathrm{H}-2$ & $67.0-69.0$ & 110.87 & MAG & $22 \mathrm{~A}$ & 255.8 & -79.8 & 4.9331 & $\mathrm{~N}$ \\
\hline $13 \mathrm{H}-2$ & $136.0-138.0$ & 111.56 & MAG & $00 \mathrm{~A}$ & 269.1 & -61.7 & 12.2000 & $\mathrm{~N}$ \\
\hline $13 \mathrm{H}-2$ & $136.0-138.0$ & 111.56 & MAG & $15 \mathrm{~A}$ & 279.3 & -69.8 & 10.8272 & $\mathrm{~N}$ \\
\hline $13 \mathrm{H}-2$ & $136.0-138.0$ & 111.56 & MAG & $22 \mathrm{~A}$ & 281.7 & -70.2 & 5.0118 & $\mathrm{~N}$ \\
\hline $13 \mathrm{H}-3$ & $37.0-39.0$ & 112.07 & MAG & $00 \mathrm{~A}$ & 269.9 & +63.4 & 3.2900 & $\mathbf{R}$ \\
\hline $13 \mathrm{H}-3$ & $37.0-39.0$ & 112.07 & MAG & $15 \mathrm{~A}$ & 212.3 & +58.4 & 3.9058 & $\mathbf{R}$ \\
\hline $13 \mathrm{H}-3$ & $37.0-39.0$ & 112.07 & MAG & $22 \mathrm{~A}$ & 175.0 & +78.5 & 2.8571 & $\mathbf{R}$ \\
\hline $13 \mathrm{H}-3$ & $88.0-90.0$ & 112.58 & MAG & $00 \mathrm{~A}$ & 270.4 & +67.5 & 6.8800 & $\mathbf{R}$ \\
\hline $13 \mathrm{H}-3$ & $88.0-90.0$ & 112.58 & MAG & $15 \mathrm{~A}$ & 209.7 & +70.7 & 7.6274 & $\mathbf{R}$ \\
\hline
\end{tabular}


Appendix (continued).

\begin{tabular}{|c|c|c|c|c|c|c|c|c|}
\hline Core, section & $\begin{array}{c}\text { Interval } \\
(\mathrm{cm})\end{array}$ & $\begin{array}{l}\text { Depth } \\
\text { (mbsf) }\end{array}$ & S.T. & Demag level & Decl. & Incl. & Intensity & Polarity \\
\hline \multicolumn{9}{|l|}{ 119-744A-(Cont.) } \\
\hline $13 \mathrm{H}-3$ & $88.0-90.0$ & 112.58 & MAG & $22 \mathrm{~A}$ & 158.1 & +78.6 & 5.3850 & $\mathbf{R}$ \\
\hline $13 \mathrm{H}-3$ & $133.0-135.0$ & 113.03 & MAG & $22 \mathrm{~A}$ & 156.5 & +70.0 & 9.1766 & $\mathbf{R}$ \\
\hline $13 \mathrm{H}-3$ & $133.0-135.0$ & 113.03 & MAG & $00 \mathrm{~A}$ & 272.8 & +84.1 & 10.7000 & $\mathbf{R}$ \\
\hline $13 \mathrm{H}-3$ & $133.0-135.0$ & 113.03 & MAG & $15 \mathrm{~A}$ & 174.8 & +71.6 & 13.1567 & $\mathbf{R}$ \\
\hline $13 \mathrm{H}-4$ & $14.0-16.0$ & 113.34 & MAG & $00 \mathrm{~A}$ & 270.7 & +65.0 & 7.6100 & $\mathbf{R}$ \\
\hline $13 \mathrm{H}-4$ & $14.0-16.0$ & 113.34 & MAG & $15 \mathrm{~A}$ & 211.9 & +73.3 & 8.3007 & $\mathbf{R}$ \\
\hline $13 \mathrm{H}-4$ & $14.0-16.0$ & 113.34 & MAG & $22 \mathrm{~A}$ & 181.4 & +81.3 & 5.6015 & $\mathbf{R}$ \\
\hline $13 \mathrm{H}-4$ & $60.0-62.0$ & 113.80 & MAG & $00 \mathrm{~A}$ & 270.6 & +74.5 & 7.8500 & $\mathbf{R}$ \\
\hline $13 \mathrm{H}-4$ & $60.0-62.0$ & 113.80 & MAG & $15 \mathrm{~A}$ & 201.2 & +66.8 & 9.2063 & $\mathbf{R}$ \\
\hline $13 \mathrm{H}-4$ & $60.0-62.0$ & 113.80 & MAG & $22 \mathrm{~A}$ & 168.7 & +71.0 & 6.7177 & $\mathbf{R}$ \\
\hline $13 \mathrm{H}-4$ & $113.0-115.0$ & 114.33 & MAG & $00 \mathrm{~A}$ & 270.5 & +55.7 & 2.9000 & $\mathbf{R}$ \\
\hline $13 \mathrm{H}-4$ & $113.0-115.0$ & 114.33 & MAG & $15 \mathrm{~A}$ & 204.8 & +61.8 & 4.0285 & $\mathrm{R}$ \\
\hline $13 \mathrm{H}-4$ & $113.0-115.0$ & 114.33 & MAG & $22 \mathrm{~A}$ & 180.6 & +75.7 & 2.6546 & $\mathbf{R}$ \\
\hline $13 \mathrm{H}-5$ & $31.0-33.0$ & 115.01 & MAG & $00 \mathrm{~A}$ & 269.8 & +50.1 & 3.4500 & $\mathbf{R}$ \\
\hline $13 \mathrm{H}-5$ & $31.0-33.0$ & 115.01 & MAG & $15 \mathrm{~A}$ & 209.0 & +63.7 & 4.1954 & $\mathbf{R}$ \\
\hline $13 \mathrm{H}-5$ & $31.0-33.0$ & 115.01 & MAG & $22 \mathrm{~A}$ & 175.3 & +77.0 & 3.2547 & $\mathrm{R}$ \\
\hline $13 \mathrm{H}-5$ & $88.0-90.0$ & 115.58 & MAG & $00 \mathrm{~A}$ & 269.4 & -38.8 & 7.5100 & $\mathrm{~N}$ \\
\hline $13 \mathrm{H}-5$ & $88.0-90.0$ & 115.58 & MAG & $15 \mathrm{~A}$ & 300.6 & -60.4 & 7.7850 & $\mathrm{~N}$ \\
\hline $13 \mathrm{H}-5$ & $88.0-90.0$ & 115.58 & MAG & $22 \mathrm{~A}$ & 317.9 & -68.4 & 3.1127 & $\mathrm{~N}$ \\
\hline \multicolumn{9}{|l|}{ 119-744B- } \\
\hline $13 \mathrm{H}-5$ & $138.0-140.0$ & 116.08 & MAG & $00 \mathrm{~A}$ & 299.3 & -53.8 & 9.9728 & $\mathrm{~N}$ \\
\hline $13 \mathrm{H}-5$ & $138.0-140.0$ & 116.08 & MAG & $10 \mathrm{~A}$ & 283.1 & -71.4 & 7.4959 & $\mathbf{N}$ \\
\hline $13 \mathrm{H}-6$ & $25.0-27.0$ & 116.45 & MAG & $00 \mathrm{~A}$ & 299.7 & -61.5 & 15.9000 & $\mathbf{N}$ \\
\hline $13 \mathrm{H}-6$ & $25.0-27.0$ & 116.45 & MAG & $15 \mathrm{~A}$ & 296.3 & -71.4 & 12.9810 & $\mathrm{~N}$ \\
\hline $13 \mathrm{H}-6$ & $25.0-27.0$ & 116.45 & MAG & $22 \mathrm{~A}$ & 300.3 & -76.4 & 4.8601 & $\mathrm{~N}$ \\
\hline $13 \mathrm{H}-6$ & $84.0-86.0$ & 117.04 & MAG & $00 \mathrm{~A}$ & 297.1 & -58.5 & 13.1000 & $\mathrm{~N}$ \\
\hline $13 \mathrm{H}-6$ & $84.0-86.0$ & 117.04 & MAG & $15 \mathrm{~A}$ & 295.0 & -65.5 & 11.7141 & $\mathbf{N}$ \\
\hline $13 \mathrm{H}-6$ & $84.0-86.0$ & 117.04 & MAG & $22 \mathrm{~A}$ & 312.5 & -66.8 & 4.1013 & $\mathrm{~N}$ \\
\hline $13 \mathrm{H}-6$ & $125.0-127.0$ & 117.45 & MAG & $00 \mathrm{~A}$ & 355.0 & -70.0 & 15.8000 & $\mathrm{~N}$ \\
\hline $13 \mathrm{H}-6$ & $125.0-127.0$ & 117.45 & MAG & $15 \mathrm{~A}$ & 336.9 & -72.7 & 14.6453 & $\mathrm{~N}$ \\
\hline $13 \mathrm{H}-6$ & $125.0-127.0$ & 117.45 & MAG & $22 \mathrm{~A}$ & 330.3 & -68.4 & 5.8346 & $\mathrm{~N}$ \\
\hline $13 \mathrm{H}-7$ & $15.0-17.0$ & 117.85 & MAG & $00 \mathrm{~A}$ & 318.0 & -61.1 & 16.5000 & $\mathrm{~N}$ \\
\hline $13 \mathrm{H}-7$ & $15.0-17.0$ & 117.85 & MAG & $15 \mathrm{~A}$ & 329.0 & -68.0 & 14.3265 & $\mathrm{~N}$ \\
\hline $13 \mathrm{H}-7$ & $15.0-17.0$ & 117.85 & MAG & $22 \mathrm{~A}$ & 330.6 & -70.8 & 6.0849 & $\mathrm{~N}$ \\
\hline $13 \mathrm{H}-\mathrm{C}$ & $18.0-20.0$ & 118.29 & MAG & $00 \mathrm{~A}$ & 219.8 & -85.3 & 14.8000 & $\mathrm{~N}$ \\
\hline $13 \mathrm{H}-\mathrm{C}$ & $18.0-20.0$ & 118.29 & MAG & $15 \mathrm{~A}$ & 153.6 & -86.3 & 14.9591 & $\mathrm{~N}$ \\
\hline $13 \mathrm{H}-\mathrm{C}$ & $18.0-20.0$ & 118.29 & MAG & $22 \mathrm{~A}$ & 82.0 & -85.4 & 6.1575 & $\mathbf{N}$ \\
\hline $14 \mathrm{H}-1$ & $25.0-27.0$ & 118.45 & MAG & $00 \mathrm{~A}$ & 129.8 & +76.3 & 8.0600 & $\mathbf{R}$ \\
\hline $14 \mathrm{H}-1$ & $25.0-27.0$ & 118.45 & MAG & $15 \mathrm{~A}$ & 304.6 & +81.7 & 7.7309 & $\mathbf{R}$ \\
\hline $14 \mathrm{H}-1$ & $25.0-27.0$ & 118.45 & MAG & $22 \mathrm{~A}$ & 288.2 & +73.5 & 4.7781 & $\mathbf{R}$ \\
\hline $14 \mathrm{H}-1$ & $59.0-61.0$ & 118.79 & MAG & $00 \mathrm{~A}$ & 127.9 & +73.8 & 4.7200 & $\mathbf{R}$ \\
\hline $14 \mathrm{H}-1$ & $59.0-61.0$ & 118.79 & MAG & $15 \mathrm{~A}$ & 7.3 & +79.3 & 3.7894 & $\mathbf{R}$ \\
\hline $14 \mathrm{H}-1$ & $59.0-61.0$ & 118.79 & MAG & $22 \mathrm{~A}$ & 338.9 & +78.3 & 2.4570 & $\mathbf{R}$ \\
\hline $14 \mathrm{H}-1$ & $112.0-114.0$ & 119.32 & MAG & $00 \mathrm{~A}$ & 274.7 & -75.7 & 5.2800 & A \\
\hline $14 \mathrm{H}-1$ & $112.0-114.0$ & 119.32 & MAG & $15 \mathrm{~A}$ & 307.8 & -33.5 & 1.7298 & A \\
\hline $14 \mathrm{H}-2$ & $23.0-25.0$ & 119.93 & MAG & $00 \mathrm{~A}$ & 84.5 & -49.3 & 5.9800 & $\mathrm{~N}$ \\
\hline $14 \mathrm{H}-2$ & $23.0-25.0$ & 119.93 & MAG & $15 \mathrm{~A}$ & 83.8 & -49.5 & 3.3917 & $\mathrm{~N}$ \\
\hline $14 \mathrm{H}-2$ & $23.0-25.0$ & 119.93 & MAG & $22 \mathrm{~A}$ & 99.3 & -37.1 & 0.8237 & $\mathrm{~N}$ \\
\hline $14 \mathrm{H}-2$ & $65.0-67.0$ & 120.35 & MAG & $00 \mathrm{~A}$ & 97.1 & -65.4 & 13.2000 & $\mathrm{~N}$ \\
\hline $14 \mathrm{H}-2$ & $65.0-67.0$ & 120.35 & MAG & $15 \mathrm{~A}$ & 94.0 & -69.7 & 9.1837 & $\mathrm{~N}$ \\
\hline $14 \mathrm{H}-2$ & $65.0-67.0$ & 120.35 & MAG & $22 \mathrm{~A}$ & 99.1 & -69.9 & 3.8965 & $\mathrm{~N}$ \\
\hline $14 \mathrm{H}-2$ & $126.0-128.0$ & 120.96 & MAG & $00 \mathrm{~A}$ & 300.9 & -69.4 & 17.7000 & $\mathrm{~N}$ \\
\hline $14 \mathrm{H}-2$ & $126.0-128.0$ & 120.96 & MAG & $15 \mathrm{~A}$ & 110.3 & -71.4 & 14.2743 & $\mathrm{~N}$ \\
\hline $14 \mathrm{H}-2$ & $126.0-128.0$ & 120.96 & MAG & $22 \mathrm{~A}$ & 115.3 & -73.7 & 6.3596 & $\mathrm{~N}$ \\
\hline $14 \mathrm{H}-3$ & $16.0-18.0$ & 1121.36 & MAG & $00 \mathrm{~A}$ & 120.6 & -72.6 & 13.9000 & $\mathrm{~N}$ \\
\hline $14 \mathrm{H}-3$ & $16.0-18.0$ & 121.36 & MAG & $15 \mathrm{~A}$ & 117.0 & -75.6 & 11.6634 & $\mathrm{~N}$ \\
\hline $14 \mathrm{H}-3$ & $16.0-18.0$ & 121.36 & MAG & $22 \mathrm{~A}$ & 131.5 & -78.3 & 4.8361 & $\mathrm{~N}$ \\
\hline $14 \mathrm{H}-3$ & $75.0-77.0$ & 121.95 & MAG & $00 \mathrm{~A}$ & 122.4 & -70.6 & 20.1000 & $\mathrm{~N}$ \\
\hline $14 \mathrm{H}-3$ & $75.0-77.0$ & 121.95 & MAG & $15 \mathrm{~A}$ & 122.2 & -74.9 & 14.4216 & $\mathrm{~N}$ \\
\hline $14 \mathrm{H}-3$ & $75.0-77.0$ & 121.95 & MAG & $22 \mathrm{~A}$ & 122.3 & -77.5 & 8.0487 & $\mathrm{~N}$ \\
\hline $14 \mathrm{H}-3$ & $129.0-131.0$ & 122.49 & MAG & $00 \mathrm{~A}$ & 92.8 & -78.1 & 21.9000 & $\mathrm{~N}$ \\
\hline $14 \mathrm{H}-3$ & $129.0-131.0$ & 122.49 & MAG & $15 \mathrm{~A}$ & 90.0 & -80.6 & 20.0190 & $\mathrm{~N}$ \\
\hline $14 \mathrm{H}-3$ & $129.0-131.0$ & 122.49 & MAG & $22 \mathrm{~A}$ & 113.8 & -82.4 & 9.2493 & $\mathrm{~N}$ \\
\hline $14 \mathrm{H}-4$ & $31.0-33.0$ & 1123.01 & MAG & $00 \mathrm{~A}$ & 103.4 & -64.4 & 15.8000 & $\mathrm{~N}$ \\
\hline $14 \mathrm{H}-4$ & $31.0-33.0$ & 123.01 & MAG & $15 \mathrm{~A}$ & 109.4 & -71.9 & 13.3467 & $\mathrm{~N}$ \\
\hline $14 \mathrm{H}-4$ & $31.0-33.0$ & 123.01 & MAG & $22 \mathrm{~A}$ & 179.6 & -74.5 & 140.3466 & $\mathrm{~N}$ \\
\hline $14 \mathrm{H}-4$ & $105.0-107.0$ & 123.75 & MAG & $00 \mathrm{~A}$ & 116.8 & -71.3 & 16.6000 & $\mathrm{~N}$ \\
\hline $14 \mathrm{H}-4$ & $105.0-107.0$ & 123.75 & MAG & $15 \mathrm{~A}$ & 123.1 & -72.4 & 14.4903 & $\mathrm{~N}$ \\
\hline $14 \mathrm{H}-4$ & $105.0-107.0$ & 123.75 & MAG & $22 \mathrm{~A}$ & 131.9 & -73.1 & 6.8806 & $\mathrm{~N}$ \\
\hline $14 \mathrm{H}-4$ & $139.0-141.0$ & 124.09 & MAG & $00 \mathrm{~A}$ & 186.8 & -72.7 & 7.8100 & $\mathrm{~N}$ \\
\hline $14 \mathrm{H}-4$ & $139.0-141.0$ & 124.09 & MAG & $15 \mathrm{~A}$ & 127.4 & -66.7 & 5.8421 & $\mathrm{~N}$ \\
\hline $14 \mathrm{H}-4$ & $139.0-141.0$ & 124.09 & MAG & $22 \mathrm{~A}$ & 144.4 & -72.3 & 3.7939 & $\mathrm{~N}$ \\
\hline $14 \mathrm{H}-5$ & $10.0-12.0$ & 124.30 & MAG & $00 \mathrm{~A}$ & 104.8 & -60.5 & 10.2000 & $\mathrm{~N}$ \\
\hline $14 \mathrm{H}-5$ & $10.0-12.0$ & 124.30 & MAG & $15 \mathrm{~A}$ & 139.5 & -69.7 & 4.5436 & $\mathrm{~N}$ \\
\hline
\end{tabular}


Appendix (continued).

\begin{tabular}{|c|c|c|c|c|c|c|c|c|}
\hline Core, section & $\begin{array}{l}\text { Interval } \\
(\mathrm{cm})\end{array}$ & $\begin{array}{l}\text { Depth } \\
\text { (mbsf) }\end{array}$ & S.T. & Demag level & Decl. & Incl. & Intensity & Polarity \\
\hline \multicolumn{9}{|l|}{ 119-744B-(Cont.) } \\
\hline $14 \mathrm{H}-5$ & $10.0-12.0$ & 124.30 & MAG & $22 \mathrm{~A}$ & 126.2 & -74.0 & 2.1349 & $\mathrm{~N}$ \\
\hline $14 \mathrm{H}-5$ & $70.0-72.0$ & 124.90 & MAG & $00 \mathrm{~A}$ & 91.8 & -41.4 & 6.0300 & A \\
\hline $14 \mathrm{H}-5$ & $70.0-72.0$ & 124.90 & MAG & $15 \mathrm{~A}$ & 101.3 & +6.2 & 1.2301 & A \\
\hline $14 \mathrm{H}-5$ & $127.0-129.0$ & 125.47 & MAG & $00 \mathrm{~A}$ & 67.7 & +59.1 & 8.8300 & $\mathbf{R}$ \\
\hline $14 \mathrm{H}-5$ & $127.0-129.0$ & 125.47 & MAG & $15 \mathrm{~A}$ & 41.0 & +75.8 & 10.1619 & $\mathbf{R}$ \\
\hline $14 \mathrm{H}-5$ & $127.0-129.0$ & 125.47 & MAG & $22 \mathrm{~A}$ & 12.0 & +77.1 & 5.4637 & $\mathbf{R}$ \\
\hline $14 \mathrm{H}-6$ & $18.0-20.0$ & 125.88 & MAG & $00 \mathrm{~A}$ & 55.5 & +64.4 & 8.1300 & $\mathbf{R}$ \\
\hline $14 \mathrm{H}-6$ & $18.0-20.0$ & 125.88 & MAG & $15 \mathrm{~A}$ & 37.1 & +71.5 & 9.3892 & $\mathbf{R}$ \\
\hline $14 \mathrm{H}-6$ & $18.0-20.0$ & 125.88 & MAG & $22 \mathrm{~A}$ & 357.4 & +80.3 & 6.5985 & $\mathbf{R}$ \\
\hline $14 \mathrm{H}-6$ & $55.0-57.0$ & 126.25 & MAG & $00 \mathrm{~A}$ & 84.8 & +30.3 & 4.0100 & $\mathbf{R}$ \\
\hline $14 \mathrm{H}-6$ & $55.0-57.0$ & 126.25 & MAG & $15 \mathrm{~A}$ & 67.5 & +56.9 & 4.6280 & $\mathbf{R}$ \\
\hline $14 \mathrm{H}-6$ & $55.0-57.0$ & 126.25 & MAG & $22 \mathrm{~A}$ & 356.9 & +83.2 & 3.1951 & $\mathbf{R}$ \\
\hline $14 \mathrm{H}-6$ & $124.0-126.0$ & 126.94 & MAG & $00 \mathrm{~A}$ & 54.6 & +28.6 & 6.1900 & $\mathbf{R}$ \\
\hline $14 \mathrm{H}-6$ & $124.0-126.0$ & 126.94 & MAG & $15 \mathrm{~A}$ & 44.4 & +56.0 & 6.8962 & $\mathbf{R}$ \\
\hline $14 \mathrm{H}-6$ & $124.0-126.0$ & 126.94 & MAG & $22 \mathrm{~A}$ & 15.7 & +73.3 & 4.6278 & $\mathbf{R}$ \\
\hline $14 \mathrm{H}-7$ & $8.0-10.0$ & 127.28 & MAG & $00 \mathrm{~A}$ & 55.0 & +20.5 & 5.9200 & $\mathbf{R}$ \\
\hline $14 \mathrm{H}-7$ & $8.0-10.0$ & 127.28 & MAG & $15 \mathrm{~A}$ & 39.4 & +72.8 & 8.8706 & $\mathbf{R}$ \\
\hline $14 \mathrm{H}-7$ & $8.0-10.0$ & 127.28 & MAG & $22 \mathrm{~A}$ & 4.1 & +76.3 & 5.7733 & $\mathbf{R}$ \\
\hline $14 \mathrm{H}-7$ & $30.0-32.0$ & 127.50 & MAG & $00 \mathrm{~A}$ & 295.9 & +74.6 & 7.0800 & $\mathbf{R}$ \\
\hline $14 \mathrm{H}-7$ & $30.0-32.0$ & 127.50 & MAG & $15 \mathrm{~A}$ & 355.3 & +71.1 & 4.8448 & $\mathbf{R}$ \\
\hline $14 \mathrm{H}-7$ & $30.0-32.0$ & 127.50 & MAG & $22 \mathrm{~A}$ & 19.2 & +69.1 & 4.7559 & $\mathbf{R}$ \\
\hline $15 \mathrm{H}-1$ & $24.0-26.0$ & 127.94 & MAG & $000 \mathrm{~A}$ & 236.2 & +65.9 & 18.6560 & $\mathbf{R}$ \\
\hline $15 \mathrm{H}-1$ & $24.0-26.0$ & 127.94 & MAG & $050 \mathrm{~A}$ & 228.0 & +68.6 & 16.0051 & $\mathbf{R}$ \\
\hline $15 \mathrm{H}-1$ & $24.0-26.0$ & 127.94 & MAG & $100 \mathrm{~A}$ & 228.0 & +67.9 & 14.4679 & $\mathbf{R}$ \\
\hline $15 \mathrm{H}-1$ & $24.0-26.0$ & 127.94 & MAG & $300 \mathrm{~A}$ & 219.5 & +65.8 & 6.0969 & $\mathbf{R}$ \\
\hline $15 \mathrm{H}-1$ & $24.0-26.0$ & 127.94 & MAG & $400 \mathrm{~A}$ & 232.9 & +66.2 & 3.2375 & $\mathbf{R}$ \\
\hline $15 \mathrm{H}-1$ & $24.0-26.0$ & 127.94 & MAG & $500 \mathrm{~A}$ & 231.3 & +71.4 & 1.9278 & $\mathbf{R}$ \\
\hline $15 \mathrm{H}-1$ & $80.0-82.0$ & 128.50 & MAG & $000 \mathrm{~A}$ & 263.7 & +63.4 & 19.2000 & $\mathbf{R}$ \\
\hline $15 \mathrm{H}-1$ & $80.0-82.0$ & 128.50 & MAG & $150 \mathrm{~A}$ & 233.0 & +74.7 & 13.7706 & $\mathbf{R}$ \\
\hline $15 \mathrm{H}-1$ & $80.0-82.0$ & 128.50 & MAG & $225 \mathrm{~A}$ & 229.5 & +76.6 & 10.7574 & $\mathbf{R}$ \\
\hline $15 \mathrm{H}-1$ & $130.0-132.0$ & 129.00 & MAG & $000 \mathrm{~A}$ & 248.8 & +67.8 & 37.9000 & $\mathbf{R}$ \\
\hline $15 \mathrm{H}-1$ & $130.0-132.0$ & 129.00 & MAG & $150 \mathrm{~A}$ & 240.3 & +70.8 & 25.8230 & $\mathbf{R}$ \\
\hline $15 \mathrm{H}-1$ & $130.0-132.0$ & 129.00 & MAG & $225 \mathrm{~A}$ & 237.7 & +72.5 & 19.2850 & $\mathbf{R}$ \\
\hline $15 \mathrm{H}-2$ & $24.0-26.0$ & 129.44 & MAG & $000 \mathrm{~A}$ & 259.4 & +66.5 & 19.1000 & $\mathbf{R}$ \\
\hline $15 \mathrm{H}-2$ & $24.0-26.0$ & 129.44 & MAG & $050 \mathrm{~A}$ & 258.5 & 72.8 & 16.500 & $\mathbf{R}$ \\
\hline $15 \mathrm{H}-2$ & $24.0-26.0$ & 129.44 & MAG & $100 \mathrm{~A}$ & 255.5 & 73.4 & 15.000 & $\mathbf{R}$ \\
\hline $15 \mathrm{H}-2$ & $24.0-26.0$ & 129.44 & MAG & $200 \mathrm{~A}$ & 262.3 & 67.7 & 11.600 & $\mathbf{R}$ \\
\hline $15 \mathrm{H}-2$ & $24.0-26.0$ & 129.44 & MAG & $300 \mathrm{~A}$ & 268.2 & +61.9 & 7.5200 & $\mathbf{R}$ \\
\hline $15 \mathrm{H}-2$ & $24.0-26.0$ & 129.44 & MAG & $400 \mathrm{~A}$ & 303.8 & +57.7 & 3.7700 & $\mathbf{R}$ \\
\hline $15 \mathrm{H}-2$ & $24.0-26.0$ & 129.44 & MAG & $500 \mathrm{~A}$ & 211.1 & +2.8 & 15.0000 & $\mathbf{R}$ \\
\hline $15 \mathrm{H}-2$ & $80.0-82.0$ & 130.00 & MAG & $000 \mathrm{~A}$ & 258.8 & +66.2 & 15.7000 & $\mathbf{R}$ \\
\hline $15 \mathrm{H}-2$ & $80.0-82.0$ & 130.00 & MAG & $150 \mathrm{~A}$ & 225.6 & +74.4 & 10.9433 & $\mathbf{R}$ \\
\hline $15 \mathrm{H}-2$ & $80.0-82.0$ & 130.00 & MAG & $225 \mathrm{~A}$ & 230.6 & +76.0 & 8.3994 & $\mathbf{R}$ \\
\hline $15 \mathrm{H}-2$ & $130.0-132.0$ & 130.50 & MAG & $000 \mathrm{~A}$ & 256.6 & +68.9 & 17.7000 & $\mathbf{R}$ \\
\hline $15 \mathrm{H}-2$ & $130.0-132.0$ & 130.50 & MAG & $150 \mathrm{~A}$ & 207.7 & +78.1 & 12.2321 & $\mathbf{R}$ \\
\hline $15 \mathrm{H}-2$ & $130.0-132.0$ & 130.50 & MAG & $225 \mathrm{~A}$ & 226.7 & +78.2 & 9.0448 & $\mathbf{R}$ \\
\hline $15 \mathrm{H}-3$ & $24.0-26.0$ & 130.94 & MAG & $000 \mathrm{~A}$ & 262.0 & +71.6 & 15.0000 & $\mathbf{R}$ \\
\hline $15 \mathrm{H}-3$ & $24.0-26.0$ & 130.94 & MAG & $150 \mathrm{~A}$ & 224.3 & +80.6 & 11.0353 & $\mathbf{R}$ \\
\hline $15 \mathrm{H}-3$ & $24.0-26.0$ & 130.94 & MAG & $225 \mathrm{~A}$ & 229.3 & +80.2 & 8.0796 & $\mathbf{R}$ \\
\hline $15 \mathrm{H}-3$ & $80.0-82.0$ & 131.50 & MAG & $000 \mathrm{~A}$ & 256.1 & +70.8 & 30.0000 & $\mathbf{R}$ \\
\hline $15 \mathrm{H}-3$ & $80.0-82.0$ & 131.50 & MAG & $150 \mathrm{~A}$ & 240.6 & +74.3 & 23.6637 & $\mathbf{R}$ \\
\hline $15 \mathrm{H}-3$ & $80.0-82.0$ & 131.50 & MAG & $225 \mathrm{~A}$ & 236.4 & +74.7 & 17.5081 & $\mathbf{R}$ \\
\hline $15 \mathrm{H}-3$ & $130.0-132.0$ & 132.00 & MAG & $000 \mathrm{~A}$ & 257.2 & +71.6 & 28.1000 & $\mathbf{R}$ \\
\hline $15 \mathrm{H}-3$ & $130.0-132.0$ & 132.00 & MAG & $150 \mathrm{~A}$ & 241.9 & +78.2 & 19.8168 & $\mathbf{R}$ \\
\hline $15 \mathrm{H}-3$ & $130.0-132.0$ & 132.00 & MAG & $225 \mathrm{~A}$ & 235.2 & +78.2 & 14.4784 & $\mathbf{R}$ \\
\hline $15 \mathrm{H}-4$ & $24.0-26.0$ & 132.44 & MAG & $000 \mathrm{~A}$ & 253.6 & +71.0 & 22.9000 & $\mathbf{R}$ \\
\hline $15 \mathrm{H}-4$ & $24.0-26.0$ & 132.44 & MAG & $150 \mathrm{~A}$ & 226.9 & +77.0 & 15.6671 & $\mathbf{R}$ \\
\hline $15 \mathrm{H}-4$ & $24.0-26.0$ & 132.44 & MAG & $225 \mathrm{~A}$ & 224.2 & +76.3 & 11.3636 & $\mathbf{R}$ \\
\hline $15 \mathrm{H}-4$ & $80.0-82.0$ & 133.00 & MAG & $000 \mathrm{~A}$ & 253.1 & +72.9 & 19.3000 & $\mathbf{R}$ \\
\hline $15 \mathrm{H}-4$ & $80.0-82.0$ & 133.00 & MAG & $150 \mathrm{~A}$ & 218.6 & +81.6 & 13.6315 & $\mathbf{R}$ \\
\hline $15 \mathrm{H}-4$ & $80.0-82.0$ & 133.00 & MAG & $225 \mathrm{~A}$ & 224.6 & +79.9 & 9.9980 & $\mathbf{R}$ \\
\hline $15 \mathrm{H}-4$ & $130.0-132.0$ & 133.50 & MAG & $000 \mathrm{~A}$ & 254.8 & +68.5 & 23.8000 & $\mathbf{R}$ \\
\hline $15 \mathrm{H}-4$ & $130.0-132.0$ & 133.50 & MAG & $150 \mathrm{~A}$ & 235.3 & +75.1 & 16.5022 & $\mathbf{R}$ \\
\hline $15 \mathrm{H}-4$ & $130.0-132.0$ & 133.50 & MAG & $225 \mathrm{~A}$ & 237.7 & +75.2 & 11.9874 & $\mathbf{R}$ \\
\hline $15 \mathrm{H}-5$ & $24.0-26.0$ & 133.94 & MAG & $000 \mathrm{~A}$ & 277.3 & +72.6 & 33.0000 & $\mathbf{R}$ \\
\hline $15 \mathrm{H}-5$ & $24.0-26.0$ & 133.94 & MAG & $150 \mathrm{~A}$ & 261.1 & +78.7 & 23.7652 & $\mathbf{R}$ \\
\hline $15 \mathrm{H}-5$ & $24.0-26.0$ & 133.94 & MAG & $225 \mathrm{~A}$ & 264.5 & +79.4 & 17.2285 & $\mathbf{R}$ \\
\hline $15 \mathrm{H}-5$ & $80.0-82.0$ & 134.50 & MAG & $000 \mathrm{~A}$ & 249.1 & +70.3 & 18.3000 & $\mathbf{R}$ \\
\hline $15 \mathrm{H}-5$ & $80.0-82.0$ & 134.50 & MAG & $150 \mathrm{~A}$ & 228.8 & +76.0 & 12.7991 & $\mathbf{R}$ \\
\hline $15 \mathrm{H}-5$ & $80.0-82.0$ & 134.50 & MAG & $225 \mathrm{~A}$ & 226.5 & +75.2 & 9.4239 & $\mathbf{R}$ \\
\hline $15 \mathrm{H}-5$ & $130.0-132.0$ & 135.00 & MAG & $000 \mathrm{~A}$ & 253.0 & +67.1 & 20.6000 & $\mathbf{R}$ \\
\hline $15 \mathrm{H}-5$ & $130.0-132.0$ & 135.00 & MAG & $150 \mathrm{~A}$ & 239.2 & +75.1 & 14.2566 & $\mathbf{R}$ \\
\hline $15 \mathrm{H}-5$ & $130.0-132.0$ & 135.00 & MAG & $225 \mathrm{~A}$ & 235.0 & +74.9 & 10.4444 & $\mathbf{R}$ \\
\hline $15 \mathrm{H}-6$ & $24.0-26.0$ & 135.44 & MAG & $000 \mathrm{~A}$ & 95.9 & +68.2 & 9.1800 & $\mathbf{R}$ \\
\hline $15 \mathrm{H}-6$ & $24.0-26.0$ & 135.44 & MAG & $150 \mathrm{~A}$ & 183.6 & +81.7 & 9.1041 & $\mathbf{R}$ \\
\hline $15 \mathrm{H}-6$ & $24.0-26.0$ & 135.44 & MAG & $225 \mathrm{~A}$ & 179.5 & +84.3 & 6.7154 & $\mathbf{R}$ \\
\hline
\end{tabular}


Appendix (continued).

\begin{tabular}{|c|c|c|c|c|c|c|c|c|}
\hline Core, section & $\begin{array}{c}\text { Interval } \\
(\mathrm{cm})\end{array}$ & $\begin{array}{l}\text { Depth } \\
\text { (mbsf) }\end{array}$ & S.T. & Demag level & Decl. & Incl. & Intensity & Polarity \\
\hline \multicolumn{9}{|l|}{ 119-744B-(Cont.) } \\
\hline $15 \mathrm{H}-6$ & $80.0-82.0$ & 136.00 & MAG & $000 \mathrm{~A}$ & 256.0 & +68.6 & 24.8000 & $\mathbf{R}$ \\
\hline $15 \mathrm{H}-6$ & $80.0-82.0$ & 136.00 & MAG & $150 \mathrm{~A}$ & 232.9 & +75.9 & 16.3676 & $\mathbf{R}$ \\
\hline $15 \mathrm{H}-6$ & $80.0-82.0$ & 136.00 & MAG & $225 \mathrm{~A}$ & 230.0 & +76.5 & 12.1063 & $\mathbf{R}$ \\
\hline $15 \mathrm{H}-6$ & $130.0-132.0$ & 136.50 & MAG & $000 \mathrm{~A}$ & 275.1 & +63.1 & 18.3000 & $\mathbf{R}$ \\
\hline $15 \mathrm{H}-6$ & $130.0-132.0$ & 136.50 & MAG & $150 \mathrm{~A}$ & 230.8 & +74.2 & 11.9577 & $\mathbf{R}$ \\
\hline $15 \mathrm{H}-6$ & $130.0-132.0$ & 136.50 & MAG & $225 \mathrm{~A}$ & 235.2 & +74.2 & 9.1215 & $\mathbf{R}$ \\
\hline $15 \mathrm{H}-7$ & $24.0-26.0$ & 136.94 & MAG & $000 \mathrm{~A}$ & 275.8 & +69.6 & 20.3000 & $\mathbf{R}$ \\
\hline $15 \mathrm{H}-7$ & $24.0-26.0$ & 136.94 & MAG & $150 \mathrm{~A}$ & 246.4 & +81.4 & 11.5161 & $\mathbf{R}$ \\
\hline $15 \mathrm{H}-7$ & $24.0-26.0$ & 136.94 & MAG & $225 \mathrm{~A}$ & 264.3 & +74.0 & 9.4454 & $\mathbf{R}$ \\
\hline $15 \mathrm{H}-7$ & $56.0-58.0$ & 137.26 & MAG & $000 \mathrm{~A}$ & 247.4 & +68.3 & 16.6000 & $\mathbf{R}$ \\
\hline $15 \mathrm{H}-7$ & $56.0-58.0$ & 137.26 & MAG & $150 \mathrm{~A}$ & 205.9 & +78.6 & 10.4439 & $\mathbf{R}$ \\
\hline $15 \mathrm{H}-7$ & $56.0-58.0$ & 137.26 & MAG & $225 \mathrm{~A}$ & 205.5 & +81.6 & 7.9346 & $\mathbf{R}$ \\
\hline $16 \mathrm{H}-1$ & $30.0-32.0$ & 137.50 & MAG & $000 \mathrm{~A}$ & 259.7 & +79.8 & 13.2000 & $\mathbf{R}$ \\
\hline $16 \mathrm{H}-1$ & $30.0-32.0$ & 137.50 & MAG & $150 \mathrm{~A}$ & 96.4 & +83.9 & 10.3035 & $\mathbf{R}$ \\
\hline $16 \mathrm{H}-1$ & $30.0-32.0$ & 137.50 & MAG & $225 \mathrm{~A}$ & 90.7 & +78.6 & 7.5152 & $\mathbf{R}$ \\
\hline $16 \mathrm{H}-1$ & $80.0-82.0$ & 138.00 & MAG & $000 \mathrm{~A}$ & 252.3 & +56.5 & 8.6000 & $\mathbf{R}$ \\
\hline $16 \mathrm{H}-1$ & $80.0-82.0$ & 138.00 & MAG & $150 \mathrm{~A}$ & 137.4 & +86.5 & 7.0448 & $\mathbf{R}$ \\
\hline $16 \mathrm{H}-1$ & $80.0-82.0$ & 138.00 & MAG & $225 \mathrm{~A}$ & 149.6 & +71.0 & 6.7800 & $\mathbf{R}$ \\
\hline $16 \mathrm{H}-1$ & $130.0-132.0$ & 138.50 & MAG & $000 \mathrm{~A}$ & 274.2 & +72.5 & 12.6000 & $\mathbf{R}$ \\
\hline $16 \mathrm{H}-1$ & $130.0-132.0$ & 138.50 & MAG & $150 \mathrm{~A}$ & 71.8 & +83.5 & 11.0284 & $\mathbf{R}$ \\
\hline $16 \mathrm{H}-1$ & $130.0-132.0$ & 138.50 & MAG & $225 \mathrm{~A}$ & 149.6 & +71.0 & 6.7786 & $\mathbf{R}$ \\
\hline $16 \mathrm{H}-2$ & $30.0-32.0$ & 139.00 & MAG & $000 \mathrm{~A}$ & 261.7 & +41.1 & 4.2900 & $\mathbf{R}$ \\
\hline $16 \mathrm{H}-2$ & $30.0-32.0$ & 139.00 & MAG & $150 \mathrm{~A}$ & 192.0 & +81.0 & 2.7628 & $\mathbf{R}$ \\
\hline $16 \mathrm{H}-2$ & $30.0-32.0$ & 139.00 & MAG & $225 \mathrm{~A}$ & 119.2 & +84.6 & 1.8970 & $\mathbf{R}$ \\
\hline $16 \mathrm{H}-2$ & $80.0-82.0$ & 139.50 & MAG & $000 \mathrm{~A}$ & 256.7 & -57.7 & 13.7000 & $\mathrm{~N}$ \\
\hline $16 \mathrm{H}-2$ & $80.0-82.0$ & 139.50 & MAG & $150 \mathrm{~A}$ & 226.6 & -76.2 & 7.6839 & $\mathrm{~N}$ \\
\hline $16 \mathrm{H}-2$ & $80.0-82.0$ & 139.50 & MAG & $225 \mathrm{~A}$ & 241.9 & -74.7 & 5.3502 & $\mathrm{~N}$ \\
\hline $16 \mathrm{H}-2$ & $130.0-132.0$ & 140.00 & MAG & $000 \mathrm{~A}$ & 250.9 & -53.7 & 16.2000 & $\mathrm{~N}$ \\
\hline $16 \mathrm{H}-2$ & $130.0-132.0$ & 140.00 & MAG & $150 \mathrm{~A}$ & 236.4 & -69.7 & 9.8575 & $\mathrm{~N}$ \\
\hline $16 \mathrm{H}-2$ & $130.0-132.0$ & 140.00 & MAG & $225 \mathrm{~A}$ & 240.2 & -69.0 & 6.9840 & $\mathrm{~N}$ \\
\hline $16 \mathrm{H}-3$ & $30.0-32.0$ & 140.50 & MAG & $000 \mathrm{~A}$ & 259.9 & -50.8 & 2.7500 & $\mathrm{~N}$ \\
\hline $16 \mathrm{H}-3$ & $30.0-32.0$ & 140.50 & MAG & $150 \mathrm{~A}$ & 272.0 & -61.3 & 8.2142 & $\mathbf{N}$ \\
\hline $16 \mathrm{H}-3$ & $30.0-32.0$ & 140.50 & MAG & $225 \mathrm{~A}$ & 268.3 & -59.2 & 6.1107 & $\mathrm{~N}$ \\
\hline $16 \mathrm{H}-3$ & $30.0-32.0$ & 140.50 & MAG & $300 \mathrm{~A}$ & 260.8 & -47.4 & 3.7714 & $\mathrm{~N}$ \\
\hline $16 \mathrm{H}-3$ & $80.0-82.0$ & 141.00 & MAG & $000 \mathrm{~A}$ & 250.2 & -41.9 & 12.1000 & $\mathrm{~N}$ \\
\hline $16 \mathrm{H}-3$ & $80.0-82.0$ & 141.00 & MAG & $150 \mathrm{~A}$ & 228.4 & -56.0 & 7.1681 & $\mathrm{~N}$ \\
\hline $16 \mathrm{H}-3$ & $80.0-82.0$ & 141.00 & MAG & $225 \mathrm{~A}$ & 234.7 & -56.8 & 5.7880 & $\mathrm{~N}$ \\
\hline $16 \mathrm{H}-3$ & $80.0-82.0$ & 141.00 & MAG & $300 \mathrm{~A}$ & 234.5 & -45.1 & 3.5879 & $\mathrm{~N}$ \\
\hline $16 \mathrm{H}-3$ & $130.0-132.0$ & 141.50 & MAG & $000 \mathrm{~A}$ & 237.8 & -38.7 & 10.5000 & $\mathrm{~N}$ \\
\hline $16 \mathrm{H}-3$ & $130.0-132.0$ & 141.50 & MAG & $150 \mathrm{~A}$ & 192.3 & -59.5 & 6.8373 & $N$ \\
\hline $16 \mathrm{H}-3$ & $130.0-132.0$ & 141.50 & MAG & $225 \mathrm{~A}$ & 206.9 & -59.2 & 5.5652 & $\mathbf{N}$ \\
\hline $16 \mathrm{H}-3$ & $130.0-132.0$ & 141.50 & MAG & $300 \mathrm{~A}$ & 215.1 & -51.7 & 4.0763 & $\mathrm{~N}$ \\
\hline $16 \mathrm{H}-4$ & $30.0-32.0$ & 142.00 & MAG & $150 \mathrm{~A}$ & 253.6 & -67.6 & 5.9259 & $\mathrm{~N}$ \\
\hline $16 \mathrm{H}-4$ & $30.0-32.0$ & 142.00 & MAG & $225 \mathrm{~A}$ & 258.5 & -69.1 & 4.4512 & $\mathrm{~N}$ \\
\hline $16 \mathrm{H}-4$ & $30.0-32.0$ & 142.00 & MAG & $300 \mathrm{~A}$ & 259.9 & -50.8 & 2.7507 & $\mathrm{~N}$ \\
\hline $16 \mathrm{H}-4$ & $80.0-82.0$ & 142.50 & MAG & $000 \mathrm{~A}$ & 238.0 & -45.5 & 12.6000 & $\mathrm{~N}$ \\
\hline $16 \mathrm{H}-4$ & $80.0-82.0$ & 142.50 & MAG & $150 \mathrm{~A}$ & 213.1 & -64.0 & 9.2027 & $\mathrm{~N}$ \\
\hline $16 \mathrm{H}-4$ & $80.0-82.0$ & 142.50 & MAG & $225 \mathrm{~A}$ & 213.3 & -65.1 & 7.5741 & $\mathrm{~N}$ \\
\hline $16 \mathrm{H}-4$ & $80.0-82.0$ & 142.50 & MAG & $300 \mathrm{~A}$ & 222.9 & -60.0 & 5.1777 & $\mathbf{N}$ \\
\hline $16 \mathrm{H}-4$ & $115.0-117.0$ & 142.85 & MAG & $000 \mathrm{~A}$ & 265.6 & +18.7 & 6.6500 & A \\
\hline $16 \mathrm{H}-4$ & $115.0-117.0$ & 142.85 & MAG & $150 \mathrm{~A}$ & 245.7 & -8.2 & 2.1508 & A \\
\hline $16 \mathrm{H}-4$ & $115.0-117.0$ & 142.85 & MAG & $225 \mathrm{~A}$ & 256.4 & -17.7 & 1.8248 & A \\
\hline $16 \mathrm{H}-4$ & $115.0-117.0$ & 142.85 & MAG & $300 \mathrm{~A}$ & 252.8 & +15.6 & 1.8974 & A \\
\hline $16 \mathrm{H}-5$ & $30.0-32.0$ & 143.50 & MAG & $000 \mathrm{~A}$ & 267.3 & +4.9 & 3.9829 & A \\
\hline $16 \mathrm{H}-5$ & $30.0-32.0$ & 143.50 & MAG & $225 \mathrm{~A}$ & 276.6 & -75.0 & 0.9382 & A \\
\hline $16 \mathrm{H}-5$ & $80.0-82.0$ & 144.00 & MAG & $000 \mathrm{~A}$ & 255.3 & -48.8 & 10.7000 & $\mathrm{~N}$ \\
\hline $16 \mathrm{H}-5$ & $80.0-82.0$ & 144.00 & MAG & $150 \mathrm{~A}$ & 228.1 & -70.5 & 7.1927 & $\mathbf{N}$ \\
\hline $16 \mathrm{H}-5$ & $80.0-82.0$ & 144.00 & MAG & $225 \mathrm{~A}$ & 236.7 & -73.0 & 5.4547 & $\mathrm{~N}$ \\
\hline $16 \mathrm{H}-5$ & $80.0-82.0$ & 144.00 & MAG & $300 \mathrm{~A}$ & 247.0 & -54.8 & 3.0398 & $\mathrm{~N}$ \\
\hline $16 \mathrm{H}-5$ & $130.0-132.0$ & 144.50 & MAG & $000 \mathrm{~A}$ & 241.1 & -37.5 & 7.8300 & $\mathbf{N}$ \\
\hline $16 \mathrm{H}-5$ & $130.0-132.0$ & 144.50 & MAG & $150 \mathrm{~A}$ & 188.4 & -64.8 & 5.7606 & $\mathrm{~N}$ \\
\hline $16 \mathrm{H}-5$ & $130.0-132.0$ & 144.50 & MAG & $225 \mathrm{~A}$ & 197.9 & -70.9 & 4.3815 & $\mathbf{N}$ \\
\hline $16 \mathrm{H}-5$ & $130.0-132.0$ & 144.50 & MAG & $300 \mathrm{~A}$ & 220.6 & -60.6 & 2.3322 & $\mathrm{~N}$ \\
\hline $16 \mathrm{H}-6$ & $30.0-32.0$ & 145.00 & MAG & $000 \mathrm{~A}$ & 269.5 & -47.0 & 9.7800 & $\mathbf{N}$ \\
\hline $16 \mathrm{H}-6$ & $30.0-32.0$ & 145.00 & MAG & $150 \mathrm{~A}$ & 261.6 & -81.0 & 4.9903 & $\mathrm{~N}$ \\
\hline $16 \mathrm{H}-6$ & $30.0-32.0$ & 145.00 & MAG & $225 \mathrm{~A}$ & 270.6 & -75.5 & 3.7804 & $\mathrm{~N}$ \\
\hline $16 \mathrm{H}-6$ & $30.0-32.0$ & 145.00 & MAG & $300 \mathrm{~A}$ & 265.9 & -57.5 & 1.8376 & $\mathbf{N}$ \\
\hline $16 \mathrm{H}-6$ & $80.0-82.0$ & 145.50 & MAG & $000 \mathrm{~A}$ & 241.3 & -52.3 & 6.8600 & $\mathbf{N}$ \\
\hline $16 \mathrm{H}-6$ & $80.0-82.0$ & 145.50 & MAG & $150 \mathrm{~A}$ & 200.8 & -75.2 & 4.2184 & $\mathbf{N}$ \\
\hline $16 \mathrm{H}-6$ & $80.0-82.0$ & 145.50 & MAG & $225 \mathrm{~A}$ & 214.7 & -72.9 & 3.1053 & $\mathrm{~N}$ \\
\hline $16 \mathrm{H}-6$ & $80.0-82.0$ & 145.50 & MAG & $300 \mathrm{~A}$ & 227.2 & -58.2 & 1.4557 & $\mathrm{~N}$ \\
\hline $16 \mathrm{H}-6$ & $130.0-132.0$ & 146.00 & MAG & $000 \mathrm{~A}$ & 248.3 & -22.1 & 4.9500 & A \\
\hline $16 \mathrm{H}-6$ & $130.0-132.0$ & 146.00 & MAG & $150 \mathrm{~A}$ & 221.6 & -69.1 & 1.3859 & A \\
\hline $16 \mathrm{H}-6$ & $130.0-132.0$ & 146.00 & MAG & $225 \mathrm{~A}$ & 228.3 & -74.0 & 1.1542 & A \\
\hline $16 \mathrm{H}-6$ & $130.0-132.0$ & 146.00 & MAG & $300 \mathrm{~A}$ & 245.2 & +9.6 & 0.7155 & A \\
\hline $16 \mathrm{H}-7$ & $30.0-32.0$ & 146.50 & MAG & $000 \mathrm{~A}$ & 267.7 & -69.0 & 16.0000 & $\mathrm{~N}$ \\
\hline
\end{tabular}


Appendix (continued).

\begin{tabular}{|c|c|c|c|c|c|c|c|c|}
\hline Core, section & $\begin{array}{c}\text { Interval } \\
(\mathrm{cm})\end{array}$ & $\begin{array}{l}\text { Depth } \\
\text { (mbsf) }\end{array}$ & S.T. & Demag level & Decl. & Incl. & Intensity & Polarity \\
\hline \multicolumn{9}{|l|}{ 119-744B-(Cont.) } \\
\hline $16 \mathrm{H}-7$ & $30.0-32.0$ & 146.50 & MAG & $150 \mathrm{~A}$ & 251.4 & -77.9 & 10.2432 & $\mathrm{~N}$ \\
\hline $16 \mathrm{H}-7$ & $30.0-32.0$ & 146.50 & MAG & $225 \mathrm{~A}$ & 246.2 & -73.1 & 8.2796 & $\mathrm{~N}$ \\
\hline $16 \mathrm{H}-7$ & $30.0-32.0$ & 146.50 & MAG & $300 \mathrm{~A}$ & 239.5 & -62.8 & 5.5489 & $\mathrm{~N}$ \\
\hline $16 \mathrm{H}-7$ & $64.0-66.0$ & 146.84 & MAG & $000 \mathrm{~A}$ & 290.8 & -42.7 & 2.7100 & $\mathrm{~N}$ \\
\hline $16 \mathrm{H}-7$ & $64.0-66.0$ & 146.84 & MAG & $150 \mathrm{~A}$ & 327.7 & -58.3 & 1.5441 & $\mathrm{~N}$ \\
\hline $16 \mathrm{H}-7$ & $64.0-66.0$ & 146.84 & MAG & $225 \mathrm{~A}$ & 292.7 & -44.6 & 1.9733 & $\mathrm{~N}$ \\
\hline $16 \mathrm{H}-7$ & $64.0-66.0$ & 146.84 & MAG & $300 \mathrm{~A}$ & 280.0 & -9.8 & 1.5890 & $\mathrm{~N}$ \\
\hline $17 \mathrm{H}-1$ & $7.0-9.0$ & 146.77 & MAG & $00 \mathrm{~A}$ & 87.0 & +0.9 & 3.8600 & $\mathbf{R}$ \\
\hline $17 \mathrm{H}-1$ & $7.0-9.0$ & 146.77 & MAG & $15 \mathrm{~A}$ & 2.4 & +46.9 & 1.0367 & $\mathbf{R}$ \\
\hline $17 \mathrm{H}-1$ & $7.0-9.0$ & 146.77 & MAG & $22 \mathrm{~A}$ & 348.8 & +62.8 & 1.3765 & $\mathbf{R}$ \\
\hline $17 \mathrm{H}-1$ & $7.0-9.0$ & 146.77 & MAG & $30 \mathrm{~A}$ & 10.2 & +68.5 & 1.2931 & $\mathbf{R}$ \\
\hline $17 \mathrm{H}-1$ & $34.0-36.0$ & 147.04 & MAG & $00 \mathrm{~A}$ & 70.7 & +54.4 & 6.6600 & $\mathbf{R}$ \\
\hline $17 \mathrm{H}-1$ & $34.0-36.0$ & 147.04 & MAG & $22 \mathrm{~A}$ & 257.6 & +82.8 & 5.8938 & $\mathrm{R}$ \\
\hline $17 \mathrm{H}-1$ & $34.0-36.0$ & 147.04 & MAG & $30 \mathrm{~A}$ & 271.9 & +78.4 & 5.0461 & $\mathbf{R}$ \\
\hline $18 \mathrm{H}-1$ & $30.0-32.0$ & 147.90 & MAG & $00 \mathrm{~A}$ & 327.3 & +1.2 & 0.1310 & A \\
\hline $18 \mathrm{H}-1$ & $30.0-32.0$ & 147.90 & MAG & $15 \mathrm{~A}$ & 143.8 & -71.1 & 1.9968 & A \\
\hline $18 \mathrm{H}-1$ & $30.0-32.0$ & 147.90 & MAG & $30 \mathrm{~A}$ & 241.8 & +49.0 & 1.4073 & A \\
\hline $18 \mathrm{H}-1$ & $80.0-82.0$ & 148.40 & MAG & $00 \mathrm{~A}$ & 86.3 & -76.9 & 5.8200 & $\mathrm{~N}$ \\
\hline $18 \mathrm{H}-\mathrm{I}$ & $80.0-82.0$ & 148.40 & MAG & $15 \mathrm{~A}$ & 136.6 & -76.7 & 3.5188 & $\mathrm{~N}$ \\
\hline $18 \mathrm{H}-1$ & $80.0-82.0$ & 148.40 & MAG & $22 \mathrm{~A}$ & 150.9 & -69.9 & 1.4506 & $\mathrm{~N}$ \\
\hline $18 \mathrm{H}-1$ & $80.0-82.0$ & 148.40 & MAG & $30 \mathrm{~A}$ & 210.0 & +37.9 & 1.1337 & $\mathrm{~N}$ \\
\hline $18 \mathrm{H}-1$ & $120.0-122.0$ & 148.80 & MAG & $15 \mathrm{~A}$ & 103.9 & -70.2 & 2.0677 & A \\
\hline $18 \mathrm{H}-1$ & $120.0-122.0$ & 148.80 & MAG & $22 \mathrm{~A}$ & 107.7 & -64.7 & 1.3484 & A \\
\hline $18 \mathrm{H}-1$ & $120.0-122.0$ & 148.80 & MAG & $30 \mathrm{~A}$ & 178.3 & +38.8 & 0.3391 & A \\
\hline $18 \mathrm{H}-2$ & $30.0-32.0$ & 149.40 & MAG & $00 \mathrm{~A}$ & 250.8 & +34.9 & 1.8100 & $\mathbf{R}$ \\
\hline $18 \mathrm{H}-2$ & $30.0-32.0$ & 149.40 & MAG & $15 \mathrm{~A}$ & 227.0 & +76.2 & 4.3771 & $\mathbf{R}$ \\
\hline $18 \mathrm{H}-2$ & $30.0-32.0$ & 149.40 & MAG & $22 \mathrm{~A}$ & 233.0 & +73.1 & 3.6545 & $\mathbf{R}$ \\
\hline $18 \mathrm{H}-2$ & $80.0-82.0$ & 149.90 & MAG & $00 \mathrm{~A}$ & 219.7 & +43.9 & 3.2200 & $\mathbf{R}$ \\
\hline $18 \mathrm{H}-2$ & $80.0-82.0$ & 149.90 & MAG & $15 \mathrm{~A}$ & 195.0 & +59.3 & 4.1090 & $\mathbf{R}$ \\
\hline $18 \mathrm{H}-2$ & $80.0-82.0$ & 149.90 & MAG & $22 \mathrm{~A}$ & 203.6 & +60.5 & 3.2787 & $\mathbf{R}$ \\
\hline $18 \mathrm{H}-2$ & $80.0-82.0$ & 149.90 & MAG & $30 \mathrm{~A}$ & 219.2 & +61.9 & 3.3561 & $\mathbf{R}$ \\
\hline $18 \mathrm{H}-2$ & $120.0-122.0$ & 150.30 & MAG & $00 \mathrm{~A}$ & 202.0 & +27.0 & 3.1500 & $\mathbf{R}$ \\
\hline $18 \mathrm{H}-2$ & $120.0-122.0$ & 150.30 & MAG & $05 \mathrm{~A}$ & 206.5 & +52.2 & 4.1500 & $\mathbf{R}$ \\
\hline $18 \mathrm{H}-2$ & $120.0-122.0$ & 150.30 & MAG & $10 \mathrm{~A}$ & 204.0 & +53.5 & 4.4000 & $\mathbf{R}$ \\
\hline $18 \mathrm{H}-2$ & $120.0-122.0$ & 150.30 & MAG & $20 \mathrm{~A}$ & 198.4 & +54.6 & 3.5800 & $\mathbf{R}$ \\
\hline $18 \mathrm{H}-2$ & $120.0-122.0$ & 150.30 & MAG & $30 \mathrm{~A}$ & 191.8 & +61.6 & 3.3100 & $\mathbf{R}$ \\
\hline $18 \mathrm{H}-2$ & $120.0-122.0$ & 150.30 & MAG & $40 \mathrm{~A}$ & 188.7 & +42.4 & 1.9100 & $\mathbf{R}$ \\
\hline $18 \mathrm{H}-2$ & $120.0-122.0$ & 150.30 & MAG & $50 \mathrm{~A}$ & 189.9 & -4.9 & 8.3300 & $\mathbf{R}$ \\
\hline $18 \mathrm{H}-3$ & $30.0-32.0$ & 150.90 & MAG & $00 \mathrm{~A}$ & 204.1 & +43.2 & 5.5300 & $\mathbf{R}$ \\
\hline $18 \mathrm{H}-3$ & $30.0-32.0$ & 150.90 & MAG & $15 \mathrm{~A}$ & 188.6 & +58.2 & 6.6431 & $\mathbf{R}$ \\
\hline $18 \mathrm{H}-3$ & $30.0-32.0$ & 150.90 & MAG & $22 \mathrm{~A}$ & 195.4 & +60.5 & 4.8880 & $\mathbf{R}$ \\
\hline $18 \mathrm{H}-3$ & $30.0-32.0$ & 150.90 & MAG & $30 \mathrm{~A}$ & 208.0 & +61.9 & 4.5401 & $\mathbf{R}$ \\
\hline $18 \mathrm{H}-3$ & $80.0-82.0$ & 151.40 & MAG & $00 \mathrm{~A}$ & 233.7 & +28.2 & 7.9300 & $\mathbf{R}$ \\
\hline $18 \mathrm{H}-3$ & $80.0-82.0$ & 151.40 & MAG & $15 \mathrm{~A}$ & 228.2 & +41.5 & 7.7216 & $\mathbf{R}$ \\
\hline $18 \mathrm{H}-3$ & $80.0-82.0$ & 151.40 & MAG & $22 \mathrm{~A}$ & 228.7 & +39.8 & 5.9559 & $\mathbf{R}$ \\
\hline $18 \mathrm{H}-3$ & $80.0-82.0$ & 151.40 & MAG & $30 \mathrm{~A}$ & 232.6 & +46.3 & 4.9467 & $\mathbf{R}$ \\
\hline $18 \mathrm{H}-3$ & $120.0-122.0$ & 151.80 & MAG & $00 \mathrm{~A}$ & 221.9 & +6.1 & 7.2600 & $\mathbf{R}$ \\
\hline $18 \mathrm{H}-3$ & $120.0-122.0$ & 151.80 & MAG & $15 \mathrm{~A}$ & 217.4 & +30.7 & 5.8032 & $\mathrm{R}$ \\
\hline $18 \mathrm{H}-3$ & $120.0-122.0$ & 151.80 & MAG & $22 \mathrm{~A}$ & 217.5 & +27.7 & 4.7347 & $\mathbf{R}$ \\
\hline $18 \mathrm{H}-3$ & $120.0-122.0$ & 151.80 & MAG & $30 \mathrm{~A}$ & 223.4 & +37.1 & 3.9711 & $\mathbf{R}$ \\
\hline $18 \mathrm{H}-4$ & $30.0-32.0$ & 152.40 & MAG & $00 \mathrm{~A}$ & 215.2 & +34.1 & 5.3100 & $\mathbf{R}$ \\
\hline $18 \mathrm{H}-4$ & $30.0-32.0$ & 152.40 & MAG & $15 \mathrm{~A}$ & 200.5 & +53.2 & 5.4826 & $\mathrm{R}$ \\
\hline $18 \mathrm{H}-4$ & $30.0-32.0$ & 152.40 & MAG & $22 \mathrm{~A}$ & 208.2 & +52.5 & 4.3111 & $\mathbf{R}$ \\
\hline $18 \mathrm{H}-4$ & $30.0-32.0$ & 152.40 & MAG & $30 \mathrm{~A}$ & 219.5 & +55.5 & 4.1437 & $\mathbf{R}$ \\
\hline $18 \mathrm{H}-4$ & $80.0-82.0$ & 152.90 & MAG & $00 \mathrm{~A}$ & 186.0 & +38.8 & 7.0900 & $\mathbf{R}$ \\
\hline $18 \mathrm{H}-4$ & $80.0-82.0$ & 152.90 & MAG & $15 \mathrm{~A}$ & 189.3 & +44.5 & 6.0900 & $\mathbf{R}$ \\
\hline $18 \mathrm{H}-4$ & $80.0-82.0$ & 152.90 & MAG & $22 \mathrm{~A}$ & 191.1 & +44.3 & 4.7796 & $\mathbf{R}$ \\
\hline $18 \mathrm{H}-4$ & $80.0-82.0$ & 152.90 & MAG & $30 \mathrm{~A}$ & 202.7 & +52.3 & 4.2598 & $\mathbf{R}$ \\
\hline $18 \mathrm{H}-4$ & $120.0-122.0$ & 153.30 & MAG & $00 \mathrm{~A}$ & 167.7 & +30.1 & 3.8500 & $\mathbf{R}$ \\
\hline $18 \mathrm{H}-4$ & $120.0-122.0$ & 153.30 & MAG & $15 \mathrm{~A}$ & 185.0 & +32.3 & 3.5623 & $\mathbf{R}$ \\
\hline $18 \mathrm{H}-4$ & $120.0-122.0$ & 153.30 & MAG & $22 \mathrm{~A}$ & 183.8 & +34.8 & 2.6321 & $\mathrm{R}$ \\
\hline $18 \mathrm{H}-4$ & $120.0-122.0$ & 153.30 & MAG & $30 \mathrm{~A}$ & 199.1 & +49.3 & 2.8871 & $\mathbf{R}$ \\
\hline $18 \mathrm{H}-5$ & $30.0-32.0$ & 153.90 & MAG & $00 \mathrm{~A}$ & 194.8 & -25.6 & 2.6000 & $\mathbf{R}$ \\
\hline $18 \mathrm{H}-5$ & $30.0-32.0$ & 153.90 & MAG & $15 \mathrm{~A}$ & 187.6 & +15.0 & 1.9404 & $\mathbf{R}$ \\
\hline $18 \mathrm{H}-5$ & $30.0-32.0$ & 153.90 & MAG & $22 \mathrm{~A}$ & 181.7 & +12.1 & 1.5968 & $\mathbf{R}$ \\
\hline $18 \mathrm{H}-5$ & $30.0-32.0$ & 153.90 & MAG & $30 \mathrm{~A}$ & 204.7 & +37.9 & 1.8679 & $\mathbf{R}$ \\
\hline $18 \mathrm{H}-5$ & $80.0-82.0$ & 154.40 & MAG & $00 \mathrm{~A}$ & 147.3 & +3.9 & 2.2600 & $\mathbf{R}$ \\
\hline $18 \mathrm{H}-5$ & $80.0-82.0$ & 154.40 & MAG & $15 \mathrm{~A}$ & 140.9 & +28.3 & 2.6822 & $\mathbf{R}$ \\
\hline $18 \mathrm{H}-5$ & $80.0-82.0$ & 154.40 & MAG & $22 \mathrm{~A}$ & 143.6 & +26.8 & 2.2722 & $\mathbf{R}$ \\
\hline $18 \mathrm{H}-5$ & $80.0-82.0$ & 154.40 & MAG & $30 \mathrm{~A}$ & 149.7 & +49.7 & 2.2229 & $\mathbf{R}$ \\
\hline $18 \mathrm{H}-5$ & $120.0-122.0$ & 154.80 & MAG & $00 \mathrm{~A}$ & 161.7 & +43.1 & 7.3100 & $\mathbf{R}$ \\
\hline $18 \mathrm{H}-5$ & $120.0-122.0$ & 154.80 & MAG & $15 \mathrm{~A}$ & 155.4 & +53.5 & 7.1888 & $\mathbf{R}$ \\
\hline $18 \mathrm{H}-5$ & $120.0-122.0$ & 154.80 & MAG & $22 \mathrm{~A}$ & 153.5 & +52.0 & 7.3051 & $\mathbf{R}$ \\
\hline $18 \mathrm{H}-5$ & $120.0-122.0$ & 154.80 & MAG & $30 \mathrm{~A}$ & 162.8 & +58.6 & 5.8769 & $\mathbf{R}$ \\
\hline $18 \mathrm{H}-6$ & $30.0-32.0$ & 155.40 & MAG & $00 \mathrm{~A}$ & 197.8 & +70.7 & 13.1000 & $\mathbf{R}$ \\
\hline $18 \mathrm{H}-6$ & $30.0-32.0$ & 155.40 & MAG & $15 \mathrm{~A}$ & 182.1 & 69.4 & 10.3886 & $\mathbf{R}$ \\
\hline
\end{tabular}


Appendix (continued).

\begin{tabular}{|c|c|c|c|c|c|c|c|c|}
\hline Core, section & $\begin{array}{c}\text { Interval } \\
(\mathrm{cm})\end{array}$ & $\begin{array}{l}\text { Depth } \\
\text { (mbsf) }\end{array}$ & S.T. & Demag level & Decl. & Incl. & Intensity & Polarity \\
\hline \multicolumn{9}{|l|}{ 119-744B-(Cont.) } \\
\hline $18 \mathrm{H}-6$ & $30.0-32.0$ & 155.40 & MAG & $22 \mathrm{~A}$ & 177.0 & +70.1 & 8.4450 & $\mathbf{R}$ \\
\hline $18 \mathrm{H}-6$ & $30.0-32.0$ & 155.40 & MAG & $30 \mathrm{~A}$ & 187.8 & +71.6 & 7.7523 & $\mathbf{R}$ \\
\hline $18 \mathrm{H}-6$ & $80.0-82.0$ & 155.90 & MAG & $00 \mathrm{~A}$ & 111.5 & -81.4 & 5.6900 & $\mathrm{~N}$ \\
\hline $18 \mathrm{H}-6$ & $80.0-82.0$ & 155.90 & MAG & $15 \mathrm{~A}$ & 105.1 & -64.0 & 6.1190 & $\mathrm{~N}$ \\
\hline $18 \mathrm{H}-6$ & $80.0-82.0$ & 155.90 & MAG & $22 \mathrm{~A}$ & 110.0 & -61.9 & 5.4695 & $\mathrm{~N}$ \\
\hline $18 \mathrm{H}-6$ & $80.0-82.0$ & 155.90 & MAG & $30 \mathrm{~A}$ & 114.1 & -63.5 & 3.5864 & $\mathrm{~N}$ \\
\hline $18 \mathrm{H}-6$ & $120.0-122.0$ & 156.30 & MAG & $00 \mathrm{~A}$ & 49.3 & +84.3 & 25.7000 & $\mathbf{R}$ \\
\hline $18 \mathrm{H}-6$ & $120.0-122.0$ & 156.30 & MAG & $15 \mathrm{~A}$ & 61.0 & +80.9 & 18.8961 & $\mathbf{R}$ \\
\hline $18 \mathrm{H}-6$ & $120.0-122.0$ & 156.30 & MAG & $22 \mathrm{~A}$ & 58.3 & +79.8 & 16.2007 & $\mathbf{R}$ \\
\hline $18 \mathrm{H}-6$ & $120.0-122.0$ & 156.30 & MAG & $30 \mathrm{~A}$ & 55.3 & +83.0 & 13.3514 & $\mathbf{R}$ \\
\hline $18 \mathrm{H}-7$ & $30.0-32.0$ & 156.90 & MAG & $00 \mathrm{~A}$ & 124.6 & -57.7 & 8.0100 & $\mathrm{~N}$ \\
\hline $18 \mathrm{H}-7$ & $30.0-32.0$ & 156.90 & MAG & $15 \mathrm{~A}$ & 124.4 & -36.7 & 5.4574 & $\mathrm{~N}$ \\
\hline $18 \mathrm{H}-7$ & $30.0-32.0$ & 156.90 & MAG & $30 \mathrm{~A}$ & 128.8 & -40.8 & 2.9626 & $\mathrm{~N}$ \\
\hline $19 \mathrm{H}-1$ & $30.0-32.0$ & 157.40 & MAG & $00 \mathrm{~A}$ & 180.4 & +73.6 & 10.6000 & $\mathbf{R}$ \\
\hline $19 \mathrm{H}-1$ & $30.0-32.0$ & 157.40 & MAG & $15 \mathrm{~A}$ & 175.9 & +70.4 & 9.4385 & $\mathrm{R}$ \\
\hline $19 \mathrm{H}-1$ & $30.0-32.0$ & 157.40 & MAG & $22 \mathrm{~A}$ & 170.9 & +71.9 & 7.5458 & $\mathbf{R}$ \\
\hline $19 \mathrm{H}-1$ & $30.0-32.0$ & 157.40 & MAG & $30 \mathrm{~A}$ & 177.8 & +72.9 & 7.2645 & $\mathbf{R}$ \\
\hline $19 \mathrm{H}-1$ & $80.0-82.0$ & 157.90 & MAG & $00 \mathrm{~A}$ & 246.4 & -69.6 & 1.6000 & $\mathrm{~N}$ \\
\hline $19 \mathrm{H}-1$ & $80.0-82.0$ & 157.90 & MAG & $15 \mathrm{~A}$ & 216.7 & -81.9 & 1.5038 & $\mathrm{~N}$ \\
\hline $19 \mathrm{H}-1$ & $80.0-82.0$ & 157.90 & MAG & $22 \mathrm{~A}$ & 234.3 & -86.3 & 1.7695 & $\mathrm{~N}$ \\
\hline $19 \mathrm{H}-1$ & $80.0-82.0$ & 157.90 & MAG & $30 \mathrm{~A}$ & 260.2 & -50.4 & 1.0149 & $\mathbf{N}$ \\
\hline $19 \mathrm{H}-1$ & $120.0-122.0$ & 158.30 & MAG & $00 \mathrm{~A}$ & 200.1 & +66.9 & 8.5400 & $\mathbf{R}$ \\
\hline $19 \mathrm{H}-1$ & $120.0-122.0$ & 158.30 & MAG & $15 \mathrm{~A}$ & 186.9 & +66.3 & 8.2357 & $\mathbf{R}$ \\
\hline $19 \mathrm{H}-1$ & $120.0-122.0$ & 158.30 & MAG & $22 \mathrm{~A}$ & 187.4 & +68.2 & 6.7940 & $\mathbf{R}$ \\
\hline $19 \mathrm{H}-1$ & $120.0-122.0$ & 158.30 & MAG & $30 \mathrm{~A}$ & 200.1 & +68.7 & 6.3402 & R \\
\hline $19 \mathrm{H}-2$ & $30.0-32.0$ & 158.90 & MAG & $00 \mathrm{~A}$ & 200.8 & +72.0 & 7.7760 & $\mathrm{R}$ \\
\hline $19 \mathrm{H}-2$ & $30.0-32.0$ & 158.90 & MAG & $15 \mathrm{~A}$ & 190.1 & +70.6 & 7.7444 & $\mathbf{R}$ \\
\hline $19 \mathrm{H}-2$ & $30.0-32.0$ & 158.90 & MAG & $22 \mathrm{~A}$ & 187.8 & +72.3 & 6.5933 & $\mathbf{R}$ \\
\hline $19 \mathrm{H}-2$ & $30.0-32.0$ & 158.90 & MAG & $30 \mathrm{~A}$ & 200.7 & +73.0 & 6.3943 & $\mathbf{R}$ \\
\hline $19 \mathrm{H}-2$ & $80.0-82.0$ & 159.40 & MAG & $00 \mathrm{~A}$ & 204.2 & +64.8 & 8.4000 & $\mathrm{R}$ \\
\hline $19 \mathrm{H}-2$ & $80.0-82.0$ & 159.40 & MAG & $05 \mathrm{~A}$ & 191.5 & +67.0 & 8.1536 & $\mathbf{R}$ \\
\hline $19 \mathrm{H}-2$ & $80.0-82.0$ & 159.40 & MAG & $10 \mathrm{~A}$ & 196.0 & +70.4 & 7.9574 & $\mathrm{R}$ \\
\hline $19 \mathrm{H}-2$ & $80.0-82.0$ & 159.40 & MAG & $20 \mathrm{~A}$ & 192.5 & $\begin{array}{r}69.2 \\
+69.2\end{array}$ & 6.5967 & $\mathbf{R}$ \\
\hline $19 \mathrm{H}-2$ & $80.0-82.0$ & 159.40 & MAG & $30 \mathrm{~A}$ & 187.9 & +68.2 & 5.2315 & $\mathbf{R}$ \\
\hline $19 \mathrm{H}-2$ & $80.0-82.0$ & 159.40 & MAG & $40 \mathrm{~A}$ & 188.9 & +67.0 & 3.9495 & $\mathbf{R}$ \\
\hline $19 \mathrm{H}-2$ & $80.0-82.0$ & 159.40 & MAG & $50 \mathrm{~A}$ & 184.3 & +65.9 & 3.5322 & $\mathbf{R}$ \\
\hline $19 \mathrm{H}-2$ & $120.0-122.0$ & 159.80 & MAG & $00 \mathrm{~A}$ & 220.8 & +61.9 & 3.2600 & $\mathbf{R}$ \\
\hline $19 \mathrm{H}-2$ & $120.0-122.0$ & 159.80 & MAG & $15 \mathrm{~A}$ & 201.5 & +63.2 & 2.9358 & $\mathbf{R}$ \\
\hline $19 \mathrm{H}-2$ & $120.0-122.0$ & 159.80 & MAG & $22 \mathrm{~A}$ & 183.6 & +62.5 & 2.2637 & $\mathbf{R}$ \\
\hline $19 \mathrm{H}-2$ & $120.0-122.0$ & 159.80 & MAG & $30 \mathrm{~A}$ & 234.7 & +64.2 & 2.8376 & $\mathbf{R}$ \\
\hline $19 \mathrm{H}-3$ & $30.0-32.0$ & 160.40 & MAG & $00 \mathrm{~A}$ & 215.7 & +54.4 & 2.3200 & $\mathbf{R}$ \\
\hline $19 \mathrm{H}-3$ & $30.0-32.0$ & 160.40 & MAG & $15 \mathrm{~A}$ & 197.1 & +67.3 & 3.4513 & $\mathbf{R}$ \\
\hline $19 \mathrm{H}-3$ & $30.0-32.0$ & 160.40 & MAG & $22 \mathrm{~A}$ & 199.6 & +67.3 & 3.2908 & $\mathbf{R}$ \\
\hline $19 \mathrm{H}-3$ & $30.0-32.0$ & 160.40 & MAG & $30 \mathrm{~A}$ & 224.0 & +70.5 & 3.7400 & $\mathrm{R}$ \\
\hline $19 \mathrm{H}-3$ & $80.0-82.0$ & 160.90 & MAG & $00 \mathrm{~A}$ & 213.2 & +66.1 & 8.0000 & $\mathrm{R}$ \\
\hline $19 \mathrm{H}-3$ & $80.0-82.0$ & 160.90 & MAG & $15 \mathrm{~A}$ & 192.2 & +68.3 & 8.3848 & $\mathbf{R}$ \\
\hline $19 \mathrm{H}-3$ & $80.0-82.0$ & 160.90 & MAG & $22 \mathrm{~A}$ & 197.1 & +69.8 & 7.8976 & $\mathbf{R}$ \\
\hline $19 \mathrm{H}-3$ & $80.0-82.0$ & 160.90 & MAG & $30 \mathrm{~A}$ & 206.2 & +69.8 & 8.0424 & $\mathbf{R}$ \\
\hline $19 \mathrm{H}-3$ & $120.0-122.0$ & 161.30 & MAG & $00 \mathrm{~A}$ & 209.8 & +52.3 & 6.7600 & $\mathbf{R}$ \\
\hline $19 \mathrm{H}-3$ & $120.0-122.0$ & 161.30 & MAG & $15 \mathrm{~A}$ & 196.1 & +61.7 & 7.4617 & $\mathbf{R}$ \\
\hline $19 \mathrm{H}-3$ & $120.0-122.0$ & 161.30 & MAG & $22 \mathrm{~A}$ & 199.8 & +66.6 & 6.2804 & $\mathbf{R}$ \\
\hline $19 \mathrm{H}-3$ & $120.0-122.0$ & 161.30 & MAG & $30 \mathrm{~A}$ & 219.6 & +66.5 & 5.9878 & $\mathrm{R}$ \\
\hline $19 \mathrm{H}-4$ & $30.0-32.0$ & 161.90 & MAG & $00 \mathrm{~A}$ & 221.8 & -78.3 & 5.3800 & $\mathrm{~N}$ \\
\hline $19 \mathrm{H}-4$ & $30.0-32.0$ & 161.90 & MAG & $15 \mathrm{~A}$ & 196.8 & -79.8 & 2.2424 & $\mathrm{~N}$ \\
\hline $19 \mathrm{H}-4$ & $30.0-32.0$ & 161.90 & MAG & $22 \mathrm{~A}$ & 99.0 & -88.9 & 1.7611 & $\mathrm{~N}$ \\
\hline $19 \mathrm{H}-4$ & $30.0-32.0$ & 161.90 & MAG & $30 \mathrm{~A}$ & 275.9 & -32.5 & 0.6082 & $\mathrm{~N}$ \\
\hline $19 \mathrm{H}-4$ & $80.0-82.0$ & 162.40 & MAG & $00 \mathrm{~A}$ & 189.9 & -67.7 & 6.0900 & $\mathrm{~N}$ \\
\hline $19 \mathrm{H}-4$ & $80.0-82.0$ & 162.40 & MAG & $15 \mathrm{~A}$ & 193.1 & -70.6 & 2.7342 & $\mathrm{~N}$ \\
\hline $19 \mathrm{H}-4$ & $80.0-82.0$ & 162.40 & MAG & $22 \mathrm{~A}$ & 195.4 & -83.2 & 2.1047 & $\mathrm{~N}$ \\
\hline $19 \mathrm{H}-4$ & $80.0-82.0$ & 162.40 & MAG & $30 \mathrm{~A}$ & 270.2 & -43.7 & 0.9403 & $\mathrm{~N}$ \\
\hline $19 \mathrm{H}-5$ & $30.0-32.0$ & 163.40 & MAG & $00 \mathrm{~A}$ & 185.4 & +60.0 & 17.6000 & R \\
\hline $19 \mathrm{H}-5$ & $30.0-32.0$ & 163.40 & MAG & $15 \mathrm{~A}$ & 180.6 & +71.0 & 15.5153 & $\mathrm{R}$ \\
\hline $19 \mathrm{H}-5$ & $30.0-32.0$ & 163.40 & MAG & $22 \mathrm{~A}$ & 182.3 & +71.0 & 10.2512 & $\mathbf{R}$ \\
\hline $19 \mathrm{H}-5$ & $30.0-32.0$ & 163.40 & MAG & $30 \mathrm{~A}$ & 188.2 & +72.8 & 6.7338 & $\mathbf{R}$ \\
\hline $19 \mathrm{H}-5$ & $80.0-82.0$ & 163.90 & MAG & $00 \mathrm{~A}$ & 207.2 & +55.7 & 13.4000 & $\mathbf{R}$ \\
\hline $19 \mathrm{H}-5$ & $80.0-82.0$ & 163.90 & MAG & $15 \mathrm{~A}$ & 207.5 & +67.0 & 1.1000 & $\mathbf{R}$ \\
\hline $19 \mathrm{H}-5$ & $80.0-82.0$ & 163.90 & MAG & $22 \mathrm{~A}$ & 213.5 & +67.6 & 7.7221 & $\mathbf{R}$ \\
\hline $19 \mathrm{H}-5$ & $80.0-82.0$ & 163.90 & MAG & $30 \mathrm{~A}$ & 221.9 & $\begin{array}{r}+68.9 \\
+\end{array}$ & 6.0570 & $\mathbf{R}$ \\
\hline $19 \mathrm{H}-5$ & $120.0-122.0$ & 164.30 & MAG & $00 \mathrm{~A}$ & 209.9 & +58.0 & 9.1800 & $\mathbf{R}$ \\
\hline $19 \mathrm{H}-5$ & $120.0-122.0$ & 164.30 & MAG & $15 \mathrm{~A}$ & 199.7 & +67.4 & 8.5305 & $\mathbf{R}$ \\
\hline $19 \mathrm{H}-5$ & $120.0-122.0$ & 164.30 & MAG & $22 \mathrm{~A}$ & 209.5 & +68.0 & 6.4138 & $\mathbf{R}$ \\
\hline $19 \mathrm{H}-5$ & $120.0-122.0$ & 164.30 & MAG & $30 \mathrm{~A}$ & 218.5 & +70.5 & 5.2565 & $\mathbf{R}$ \\
\hline $19 \mathrm{H}-6$ & $30.0-32.0$ & 164.90 & MAG & $00 \mathrm{~A}$ & 196.4 & -61.7 & 3.6200 & $\mathrm{~N}$ \\
\hline $19 \mathrm{H}-6$ & $30.0-32.0$ & 164.90 & MAG & $15 \mathrm{~A}$ & 183.8 & -48.2 & 0.8439 & $\mathrm{~N}$ \\
\hline $19 \mathrm{H}-6$ & $30.0-32.0$ & 164.90 & MAG & $22 \mathrm{~A}$ & 33.4 & -76.5 & 1.3980 & $\mathrm{~N}$ \\
\hline $19 \mathrm{H}-6$ & $30.0-32.0$ & 164.90 & MAG & $30 \mathrm{~A}$ & 315.9 & -53.1 & 0.7706 & $\mathrm{~N}$ \\
\hline
\end{tabular}


Appendix (continued).

\begin{tabular}{|c|c|c|c|c|c|c|c|c|}
\hline Core, section & $\begin{array}{l}\text { Interval } \\
(\mathrm{cm})\end{array}$ & $\begin{array}{l}\text { Depth } \\
\text { (mbsf) }\end{array}$ & S.T. & Demag level & Decl. & Incl. & Intensity & Polarity \\
\hline \multicolumn{9}{|l|}{ 119-744B-(Cont.) } \\
\hline $19 \mathrm{H}-6$ & $80.0-82.0$ & 165.40 & MAG & $00 \mathrm{~A}$ & 198.0 & +22.1 & 4.4000 & $\mathbf{R}$ \\
\hline $19 \mathrm{H}-6$ & $80.0-82.0$ & 165.40 & MAG & $22 \mathrm{~A}$ & 195.9 & +64.0 & 3.2782 & $\mathbf{R}$ \\
\hline $19 \mathrm{H}-6$ & $80.0-82.0$ & 165.40 & MAG & $30 \mathrm{~A}$ & 230.4 & +69.0 & 2.0403 & $\mathbf{R}$ \\
\hline $20 \mathrm{H}-1$ & $8.0-10.0$ & 166.68 & MAG & $00 \mathrm{~A}$ & 354.4 & -81.8 & 15.3000 & $\mathrm{~N}$ \\
\hline $20 \mathrm{H}-1$ & $8.0-10.0$ & 166.68 & MAG & $15 \mathrm{~A}$ & 4.8 & -77.7 & 13.7265 & $\mathrm{~N}$ \\
\hline $20 \mathrm{H}-1$ & $8.0-10.0$ & 166.68 & MAG & $22 \mathrm{~A}$ & 353.0 & -71.7 & 4.9497 & $\mathrm{~N}$ \\
\hline $20 \mathrm{H}-1$ & $8.0-10.0$ & 166.68 & MAG & $30 \mathrm{~A}$ & 331.2 & -53.8 & 2.1405 & $\mathrm{~N}$ \\
\hline $20 \mathrm{H}-2$ & $80.0-82.0$ & 168.90 & MAG & $00 \mathrm{~A}$ & 211.5 & +79.8 & 9.7600 & $\mathbf{R}$ \\
\hline $20 \mathrm{H}-2$ & $80.0-82.0$ & 168.90 & MAG & $15 \mathrm{~A}$ & 185.2 & +77.0 & 7.9264 & $\mathbf{R}$ \\
\hline $20 \mathrm{H}-2$ & $80.0-82.0$ & 168.90 & MAG & $22 \mathrm{~A}$ & 175.2 & +73.1 & 1.8562 & $\mathbf{R}$ \\
\hline $20 \mathrm{H}-2$ & $80.0-82.0$ & 168.90 & MAG & $30 \mathrm{~A}$ & 246.6 & +70.7 & 1.6951 & $\mathbf{R}$ \\
\hline $20 \mathrm{H}-2$ & $120.0-122.0$ & 169.30 & MAG & $00 \mathrm{~A}$ & 235.9 & -80.3 & 2.5100 & A \\
\hline $20 \mathrm{H}-2$ & $120.0-122.0$ & 169.30 & MAG & $15 \mathrm{~A}$ & 281.9 & -86.8 & 2.1243 & A \\
\hline $20 \mathrm{H}-2$ & $120.0-122.0$ & 169.30 & MAG & $30 \mathrm{~A}$ & 267.1 & +36.0 & 0.8576 & A \\
\hline $20 \mathrm{H}-3$ & $40.0-42.0$ & 170.00 & MAG & $00 \mathrm{~A}$ & 116.2 & +13.9 & 0.0229 & A \\
\hline $20 \mathrm{H}-3$ & $40.0-42.0$ & 170.00 & MAG & $15 \mathrm{~A}$ & 31.0 & -84.6 & 10.3747 & A \\
\hline $20 \mathrm{H}-3$ & $40.0-42.0$ & 170.00 & MAG & $22 \mathrm{~A}$ & 35.7 & -71.1 & 2.5843 & A \\
\hline $20 \mathrm{H}-3$ & $40.0-42.0$ & 170.00 & MAG & $30 \mathrm{~A}$ & 325.7 & -45.1 & 0.9485 & A \\
\hline $20 \mathrm{H}-3$ & $80.0-82.0$ & 170.40 & MAG & $00 \mathrm{~A}$ & 161.7 & -81.2 & 12.7000 & $\mathrm{~N}$ \\
\hline $20 \mathrm{H}-3$ & $80.0-82.0$ & 170.40 & MAG & $15 \mathrm{~A}$ & 155.2 & -83.4 & 10.8116 & $\mathrm{~N}$ \\
\hline $20 \mathrm{H}-3$ & $80.0-82.0$ & 170.40 & MAG & $22 \mathrm{~A}$ & 116.4 & -80.7 & 2.1321 & $\mathrm{~N}$ \\
\hline $20 \mathrm{H}-3$ & $80.0-82.0$ & 170.40 & MAG & $30 \mathrm{~A}$ & 258.1 & -41.1 & 0.4608 & $\mathrm{~N}$ \\
\hline $20 \mathrm{H}-3$ & $120.0-122.0$ & 170.80 & MAG & $00 \mathrm{~A}$ & 117.4 & -83.5 & 12.4000 & $\mathrm{~N}$ \\
\hline $20 \mathrm{H}-3$ & $120.0-122.0$ & 170.80 & MAG & $15 \mathrm{~A}$ & 61.0 & -85.5 & 10.1690 & $\mathrm{~N}$ \\
\hline $20 \mathrm{H}-3$ & $120.0-122.0$ & 170.80 & MAG & $22 \mathrm{~A}$ & 43.7 & -75.9 & 2.9295 & $\mathrm{~N}$ \\
\hline $20 \mathrm{H}-3$ & $120.0-122.0$ & 170.80 & MAG & $30 \mathrm{~A}$ & 342.8 & -76.5 & 0.9660 & $\mathrm{~N}$ \\
\hline $20 \mathrm{H}-4$ & $30.0-32.0$ & 171.40 & MAG & $00 \mathrm{~A}$ & 262.1 & -35.5 & 0.4600 & A \\
\hline $20 \mathrm{H}-4$ & $30.0-32.0$ & 171.40 & MAG & $15 \mathrm{~A}$ & 126.6 & -88.9 & 0.1299 & A \\
\hline $20 \mathrm{H}-4$ & $30.0-32.0$ & 171.40 & MAG & $22 \mathrm{~A}$ & 326.5 & -85.1 & 0.2403 & A \\
\hline $20 \mathrm{H}-4$ & $30.0-32.0$ & 171.40 & MAG & $30 \mathrm{~A}$ & 247.8 & +59.2 & 0.7800 & A \\
\hline $20 \mathrm{H}-4$ & $80.0-82.0$ & 171.90 & MAG & $00 \mathrm{~A}$ & 202.5 & -30.9 & 0.4550 & $\mathrm{~N}$ \\
\hline $20 \mathrm{H}-4$ & $80.0-82.0$ & 171.90 & MAG & $15 \mathrm{~A}$ & 242.7 & -7.8 & 0.1130 & $\mathrm{~N}$ \\
\hline $20 \mathrm{H}-4$ & $80.0-82.0$ & 171.90 & MAG & $22 \mathrm{~A}$ & 31.9 & -79.3 & 0.1801 & $\mathrm{~N}$ \\
\hline $20 \mathrm{H}-4$ & $80.0-82.0$ & 171.90 & MAG & $30 \mathrm{~A}$ & 259.4 & +53.4 & 0.9673 & $\mathrm{~N}$ \\
\hline $20 \mathrm{H}-4$ & $120.0-122.0$ & 172.30 & MAG & $00 \mathrm{~A}$ & 6.3 & -43.5 & 0.2070 & $\mathrm{~N}$ \\
\hline $20 \mathrm{H}-4$ & $120.0-122.0$ & 172.30 & MAG & $05 \mathrm{~A}$ & 303.4 & -46.9 & 0.4910 & $\mathrm{~N}$ \\
\hline $20 \mathrm{H}-4$ & $120.0-122.0$ & 172.30 & MAG & $10 \mathrm{~A}$ & 3.9 & -55.0 & 0.1730 & $\mathrm{~N}$ \\
\hline $20 \mathrm{H}-4$ & $120.0-122.0$ & 172.30 & MAG & $50 \mathrm{~A}$ & 133.4 & -83.7 & 0.0782 & $\mathrm{~N}$ \\
\hline $20 \mathrm{H}-5$ & $30.0-32.0$ & 172.90 & MAG & $00 \mathrm{~A}$ & 272.3 & -7.3 & 0.3070 & A \\
\hline $20 \mathrm{H}-5$ & $30.0-32.0$ & 172.90 & MAG & $15 \mathrm{~A}$ & 224.2 & -12.0 & 0.2177 & A \\
\hline $20 \mathrm{H}-5$ & $30.0-32.0$ & 172.90 & MAG & $22 \mathrm{~A}$ & 56.3 & -70.2 & 0.2046 & A \\
\hline $20 \mathrm{H}-5$ & $30.0-32.0$ & 172.90 & MAG & $30 \mathrm{~A}$ & 261.0 & +49.1 & 0.9993 & A \\
\hline $20 \mathrm{H}-5$ & $80.0-82.0$ & 173.40 & MAG & $00 \mathrm{~A}$ & 210.0 & +42.4 & 0.3040 & $\mathbf{R}$ \\
\hline $20 \mathrm{H}-5$ & $80.0-82.0$ & 173.40 & MAG & $15 \mathrm{~A}$ & 194.8 & +47.7 & 0.3061 & $\mathbf{R}$ \\
\hline $20 \mathrm{H}-5$ & $80.0-82.0$ & 173.40 & MAG & $22 \mathrm{~A}$ & 123.4 & +26.2 & 0.2190 & $\mathrm{R}$ \\
\hline $20 \mathrm{H}-5$ & $80.0-82.0$ & 173.40 & MAG & $30 \mathrm{~A}$ & 263.7 & +64.0 & 1.1011 & $\mathbf{R}$ \\
\hline $20 \mathrm{H}-5$ & $120.0-122.0$ & 173.80 & MAG & $00 \mathrm{~A}$ & 231.3 & +30.6 & 0.4950 & $\mathrm{R}$ \\
\hline $20 \mathrm{H}-5$ & $120.0-122.0$ & 173.80 & MAG & $15 \mathrm{~A}$ & 270.3 & +50.6 & 0.2406 & $\mathbf{R}$ \\
\hline $20 \mathrm{H}-5$ & $120.0-122.0$ & 173.80 & MAG & $22 \mathrm{~A}$ & 165.1 & +38.6 & 0.1449 & $\mathbf{R}$ \\
\hline $20 \mathrm{H}-5$ & $120.0-122.0$ & 173.80 & MAG & $30 \mathrm{~A}$ & 266.9 & +59.4 & 1.2208 & $\mathbf{R}$ \\
\hline $20 \mathrm{H}-6$ & $30.0-32.0$ & 174.40 & MAG & $00 \mathrm{~A}$ & 283.4 & +1.4 & 0.2940 & $\mathbf{R}$ \\
\hline $20 \mathrm{H}-6$ & $30.0-32.0$ & 174.40 & MAG & $15 \mathrm{~A}$ & 258.9 & +43.3 & 0.3547 & $\mathbf{R}$ \\
\hline $20 \mathrm{H}-6$ & $30.0-32.0$ & 174.40 & MAG & $22 \mathrm{~A}$ & 180.6 & +45.1 & 0.1269 & $\mathbf{R}$ \\
\hline $20 \mathrm{H}-6$ & $30.0-32.0$ & 174.40 & MAG & $30 \mathrm{~A}$ & 256.1 & +57.7 & 1.0859 & $\mathbf{R}$ \\
\hline $20 \mathrm{H}-6$ & $80.0-82.0$ & 174.90 & MAG & $00 \mathrm{~A}$ & 315.9 & -2.6 & 0.3820 & A \\
\hline $20 \mathrm{H}-6$ & $80.0-82.0$ & 174.90 & MAG & $15 \mathrm{~A}$ & 237.1 & +52.1 & 0.2970 & A \\
\hline $20 \mathrm{H}-6$ & $80.0-82.0$ & 174.90 & MAG & $22 \mathrm{~A}$ & 295.1 & -11.0 & 0.1180 & A \\
\hline $20 \mathrm{H}-6$ & $80.0-82.0$ & 174.90 & MAG & $30 \mathrm{~A}$ & 274.5 & +51.2 & 1.1185 & A \\
\hline $20 \mathrm{H}-6$ & $117.0-119.0$ & 175.27 & MAG & $00 \mathrm{~A}$ & 253.6 & +37.5 & 0.6040 & $\mathbf{R}$ \\
\hline $20 \mathrm{H}-6$ & $117.0-119.0$ & 175.27 & MAG & $15 \mathrm{~A}$ & 225.7 & +49.2 & 0.5454 & $\mathbf{R}$ \\
\hline $20 \mathrm{H}-6$ & $117.0-119.0$ & 175.27 & MAG & $22 \mathrm{~A}$ & 40.2 & +75.5 & 1.1200 & $\mathbf{R}$ \\
\hline $20 \mathrm{H}-6$ & $117.0-119.0$ & 175.27 & MAG & $30 \mathrm{~A}$ & 265.5 & +55.9 & 1.3367 & $\mathbf{R}$ \\
\hline $20 \mathrm{H}-7$ & $30.0-32.0$ & 175.90 & MAG & $00 \mathrm{~A}$ & 291.5 & +21.2 & 0.5700 & $\mathbf{R}$ \\
\hline $20 \mathrm{H}-7$ & $30.0-32.0$ & 175.90 & MAG & $15 \mathrm{~A}$ & 269.5 & +0.8 & 0.2501 & $\mathbf{R}$ \\
\hline $20 \mathrm{H}-7$ & $30.0-32.0$ & 175.90 & MAG & $30 \mathrm{~A}$ & 269.2 & +64.5 & 1.1412 & $\mathbf{R}$ \\
\hline
\end{tabular}

